\title{
The Term Structure of Interbank Risk
}

\author{
Damir Filipović and Anders B. Trolle \\ Ecole Polytechnique Fédérale de Lausanne and Swiss Finance Institute
}

\begin{abstract}
We infer a term structure of interbank risk from spreads between rates on interest rate swaps indexed to LIBOR and overnight indexed swaps. We develop a model of interbank risk to decompose the term structure into default and non-default (liquidity) components. We find that, on average, from August 2007 to January 2011, the fraction of total interbank risk due to default risk increases with maturity. At the short end of the term structure, the non-default component is important in the first half of the sample period and is correlated with measures of funding and market liquidity. At longer maturities, the default component is the dominant driver of interbank risk throughout the sample period. Results hold true in both the USD and EUR markets and are robust to different model parameterizations and measures of interbank default risk. The analysis has implications for monetary and regulatory policy and for pricing, hedging, and risk-management in swap markets.
\end{abstract}

JEL Classification: E43, G12

Keywords: interbank risk, swap market, default risk, liquidity

\section{CONTAINS ONLINE APPENDIX}

This version: April 2012

\begin{abstract}
We thank Pierre Collin-Dufresne, Darrell Duffie, Rudiger Fahlenbrach, Peter Feldhutter, Michael Fleming, Masaaki Fujii, Joao Gomes, Holger Kraft, David Lando, Jesper Lund, Erwan Morellec, Claus Munk, Alberto Plazzi, Olivier Scalliet, Marco Taboga, Christian Upper, and seminar participants at the Conference on Liquidity and Credit Risk in Freiburg, the Conference on Mathematical Modeling of Systemic Risk in Paris, the 2011 Chemnitz workshop, the 2011 CREDIT conference in Venice, the 2011 FINRISK research day, the 2012 workshop on Quantitative Risk Management in Oberwolfach, ETH Zurich, Goethe University in Frankfurt, University of Lisbon (NOVA), University of Southern Denmark, University of St. Gallen, University of Tokyo, University of Zurich, and the EPFL-UNIL brownbag for comments. Shadi Akiki provided excellent research assistance. Both authors gratefully acknowledge research support from NCCR FINRISK of the Swiss National Science Foundation. E-mails: damir.filipovic@epfl.ch and anders.trolle@epfl.ch.
\end{abstract}


"The age of innocence - when banks lent to each other unsecured for three months or longer at only a small premium to expected policy rates - will not quickly, if ever, return".

Mervin King, Bank of England Governor, 21 October 2008

\section{Introduction}

Interbank risk, as defined in this paper, is the risk of direct or indirect loss resulting from lending in the interbank money market. The recent financial crisis has highlighted the implications of such risk for financial markets and economic growth. While existing studies have provided important insights on the determinants of short term interbank risk, we still know very little about the term structure of interbank risk. In this paper, we provide a comprehensive analysis of this topic. First, we develop a model of the term structure of interbank risk. Second, we apply the model to analyze interbank risk since the onset of the financial crisis, decomposing the term structure of interbank risk into default and non-default (liquidity) components.

To illustrate the importance of the term structure dimension for understanding interbank risk, consider Figure 1. The solid line shows the spread between 3M LIBOR, which is a reference rate for unsecured interbank borrowing and lending, and the fixed rate on a 3M overnight indexed swap (OIS), which is a common risk-free rate proxy. This money market spread is used in many papers and in the financial press as a measure of interbank risk. The dotted line shows the spread between the fixed rate on a 5Y regular interest rate swap (IRS) with floating-leg payments indexed to 3M LIBOR, and the fixed rate on a $5 \mathrm{Y}$ OIS. We show in the paper that this spread essentially reflects expectations about future 3M LIBOR-OIS spreads and, therefore, provides valuable insights into market participants' perceptions about future interbank risk. As such, we can use IRS-OIS spreads at different maturities to infer a term structure of interbank risk.

Prior to the onset of the credit crisis, the term structure of interbank risk was essentially flat with swap spreads only a few basis points higher than money market spreads. Then, at the onset of the crisis in August 2007, money market spreads increased much more than swap spreads. This resulted in a strongly downward-sloping term structure of interbank risk, indicating that market participants expected the extremely high levels of interbank risk observed in the money market to be a relatively short-lived phenomenon. Finally, from Fall 2009 to the end of our sample period, money market spreads were more or less back to pre-crisis levels (except for a transitory increase related to the escalation of the European sovereign debt crisis), 
while swap spreads remained well above pre-crisis levels and significantly higher than money market spreads. The result was an upward-sloping term structure of interbank risk, indicating that market participants expected interbank risk to increase in the future (and/or required a large risk premium for bearing future interbank risk). The term structure, therefore, contains important information about interbank risk that is not contained in money market spreads.

A LIBOR-OIS spread can arise for several reasons. An obvious candidate is default risk. LIBOR is a benchmark indicating the average rate at which large, creditworthy banks belonging to the LIBOR panel can obtain unsecured funding for longer terms (typically $3 \mathrm{M}$ or $6 \mathrm{M})$ in the interbank money market. An OIS is a swap with floating payments based on a reference rate for unsecured overnight funding, which we assume equals the average cost of unsecured overnight funding for LIBOR panel banks. ${ }^{1}$ An important feature of the LIBOR panel is that its composition is updated over time to include only creditworthy banks - a bank that experiences a significant deterioration in its credit quality will be dropped from the panel and be replaced by a bank with superior credit quality. Therefore, the OIS rate reflects the average credit quality of a refreshed pool of creditworthy banks, while LIBOR incorporates the risk that the average credit quality of an initial set of creditworthy banks will deteriorate over the term of the loan. ${ }^{2}$ Consequently, LIBOR exceeds the OIS rate.

To formalize this, we set up a model where, at a given point in time, we distinguish between the average default intensity of the periodically refreshed panel and the default intensity of an average bank within an initial panel. Deterioration in the credit quality of this bank relative to the average credit quality of the periodically refreshed panel occurs according to a jump process. The first jump time is interpreted as the time when the bank is dropped from the panel. The risk of credit quality deterioration (i.e., the intensity of the jump process) varies stochastically over time. In this setting, the default component of the LIBOR-OIS spread is driven by the expected credit quality deterioration of an average bank within the initial panel.

A LIBOR-OIS spread may also arise due to factors not directly related to default risk,

\footnotetext{
${ }^{1}$ While this assumption is true in the EUR market, it is only approximately true in the USD market, since the reference rate (the effective Federal Funds rate) reflects the average funding cost for a broader set of banks than the LIBOR panel. Nevertheless, Afonso, Kovner, and Schoar (2009) show that credit risk in the Federal Funds market is managed via credit rationing rather than interest rates, so that only creditworthy banks participate in the Federal Funds market. Such credit rationing was particularly prevalent in the aftermath of the Lehman default.

${ }^{2}$ We stress that there is negligible default risk in the OIS contract due to collateralization. However, the OIS rate does reflect default risk due to the indexation of the floating leg to an unsecured overnight rate.
} 
primarily liquidity. There are several reasons why liquidity in the market for longer-term interbank funding can deteriorate. For instance, banks may refrain from lending long-term for precautionary reasons, if they fear adverse shocks to their own funding situation, or for speculative reasons, if they anticipate possible fire-sales of assets by other financial institutions. ${ }^{3}$ Rather than modeling these mechanisms directly, we posit a "residual" factor that captures the component of the LIBOR-OIS spread that is not due to default risk. To the extent that liquidity effects are correlated with default risk, the residual factor captures the component of liquidity that is unspanned by default risk.

Since a long-term IRS-OIS spread reflects expectations about future short-term LIBOROIS spreads, the term structure of IRS-OIS spreads reflects the term structures of the default and non-default components of LIBOR-OIS spreads. To infer the default component, we use information from the credit default swap (CDS) market. At each observation date, we construct a CDS spread term structure for an average panel bank as a composite of the CDS spread term structures for the individual panel banks. Assuming that CDS spreads are pure measures of default risk of the underlying entities, the CDS spread term structure for an average panel bank identifies the process driving the risk of credit quality deterioration.

Our model is set within a general affine framework. Depending on the specification, two factors drive the OIS term structure, one or two factors drive the default component of LIBOROIS spreads (i.e., the risk of credit quality deterioration), and one or two factors drive the non-default component of LIBOR-OIS spreads. The model is highly tractable with analytical expressions for LIBOR, OIS, IRS, and CDS. In valuing swap contracts, we match as closely as possible current market practice regarding collateralization.

We apply the model to study interbank risk from the onset of the financial crisis in August 2007 until January 2011. We utilize a panel data set consisting of term structures of OIS rates, IRS-OIS spreads indexed to 3M and 6M LIBOR, and CDS spreads - all with maturities up to 10Y. The model is estimated by maximum likelihood in conjunction with the Kalman filter.

We conduct a specification analysis, which shows that a specification with two factors driving the OIS term structure, two factors driving the default component of the LIBOR-OIS spread, and one factor driving the non-default component of the LIBOR-OIS spread has a

\footnotetext{
${ }^{3}$ The precautionary motive for cash hoarding is modeled by Allen, Carletti, and Gale (2009) and Acharya and Skeie (2010), while the speculative motive for cash hoarding is modeled by Acharya, Gromb, and Yorulmazer (2007), Acharya, Shin, and Yorulmazer (2010) and Diamond and Rajan (2010). A recent model by Gale and Yorulmazer (2011) features both the precautionary and speculative motive for cash holdings.
} 
satisfactory fit to the data, while being fairly parsimonious. We then use this specification to decompose the term structure of interbank risk into default and non-default components. We find that, on average, the fraction of total interbank risk due to default risk increases with maturity. At the short end of the term structure, the non-default component is important in the first half of the sample period, while at longer maturities, the default component is the dominant driver of interbank risk throughout the sample period.

To understand the determinants of the non-default component of interbank risk, we relate the residual factor to a number of proxies for funding liquidity and market liquidity, which tend to be highly intertwined (Brunnermeier and Pedersen (2009)). Given its over-the-counter structure, we do not have liquidity measures specific to the unsecured interbank term funding market. Instead, we consider two liquidity measures for the related secured term funding market: the spread between 3M Agency MBS and Treasury repo rates and the liquidity factor from Fontaine and Garcia (2011). We also consider several market liquidity measures: the Treasury market liquidity factor from Hu, Pan, and Wang (2010), the Refcorp-Treasury bond yield spread suggested by Longstaff (2004), and two corporate bond market liquidity factors from Dick-Nielsen, Feldhutter, and Lando (2012). Our residual factor is significantly related to the components of the liquidity measures, which are unspanned by interbank default risk, with the $R^{2}$ reaching 64 percent in a multivariate regression specification. ${ }^{4}$ This strongly suggests that the non-default component of interbank risk largely captures liquidity effects not spanned by default risk.

We also provide tentative evidence on the pricing of interbank risk in the interest rate swap market. We find that swap market participants require compensation for exposure to variation in interbank default risk, while we are not able to reliably estimate the compensation required for exposure to the residual factor. This implies that in the first half of the sample period, when the non-default component dominates, the overall compensation for variation in interbank risk is low. In contrast, in the second half of the sample period, when the default component dominates, the overall compensation for variation in interbank risk is significant. For instance, the instantaneous Sharpe ratio of being long the 5Y IRS-OIS spread (for the IRS indexed to 3M LIBOR) is estimated to have averaged 0.35 from early 2009 to the end of the sample period.

\footnotetext{
${ }^{4}$ For each liquidity measure, the component that is unspanned by interbank default risk is given by the residual from a regression of the liquidity measure on the first two principal components of the composite CDS term structure.
} 
We perform a variety of robustness checks, which show that the results hold true for alternative model parameterizations and measures of interbank default risk. By using CDS spreads to identify the default component of interbank risk, our approach is reminiscent of Longstaff, Mithal, and Neis (2005), Blanco, Brennan, and Marsh (2005), Beber, Brandt, and Kavajecz (2009), and Ang and Longstaff (2011), among others, who use CDS spreads as pure measures of default risk. However, a number of recent papers have found that CDS spreads may be affected by liquidity effects. ${ }^{5}$ Since we mostly use CDS contracts written on large financial institutions, which are among the most liquid contracts in the CDS market, and since we aggregate individual CDS spreads, which reduces the effect of idiosyncratic noise in the individual CDS spreads, we believe it is reasonable to use the composite CDS spreads to measure default risk. Nevertheless, we consider two alternative measures of default risk that correct for possible liquidity effects. First, we measure default risk by 90 percent of the composite CDS spreads, which, given the results in Buhler and Trapp (2010), seems to be a reasonable lower bound on the default component of CDS spreads. And, second, we measure default risk by composite CDS spreads constructed solely from the banks with the most liquid CDS contracts. None of these alternative measures substantially change the decomposition of the term structure interbank risk.

We also address concerns about the integrity of LIBOR during parts of the sample period. ${ }^{6}$ Note, first, that the procedure for computing LIBOR as well as governance mechanisms intended to identify anomalous rates should minimize the impact of possible strategic behavior by certain market participants. ${ }^{7}$ Note, second, that even if LIBOR were affected, this is unlikely to impact our results, since interbank risk is primarily inferred from the cross-section of swap rates, which are determined in highly competitive markets. Instead, idiosyncratic variation in LIBOR rates will show up as a pricing error in our Kalman filter setting. Nonetheless,

\footnotetext{
${ }^{5}$ See, e.g., Buhler and Trapp (2010) and Bongaerts, de Jong, and Driessen (2011). While it is possible that CDS spreads are also affected by counterparty risk, Arora, Gandhi, and Longstaff (2009) find that this effect is minimal, which is consistent with the widespread use of collateralization and netting agreements.

${ }^{6}$ The issue is whether certain banks strategically manipulated their LIBOR quotes to signal information about their credit quality or liquidity needs or to influence LIBOR to benefit positions in LIBOR-linked instruments.

${ }^{7}$ Indeed, a Bank of International Settlements study finds that "available data do not support the hypothesis that contributor banks manipulated their quotes to profit from positions based on fixings", see Gyntelberg and Wooldridge (2008, p. 70). Likewise, an IMF study finds that "it appears that U.S. dollar LIBOR remains an accurate measure of a typical creditworthy bank's marginal cost of unsecured U.S. dollar term funding", see $\operatorname{IMF}(2008$, p. 76).
} 
as an additional check, we reestimate the model using only swap rates but find no significant changes to the results.

Throughout, we also report results for the EUR market. Not only does this serve as an additional robustness check, but this market is interesting in its own right. First, by several measures, the market is even larger than the USD market. Second, the structure of the EUR market is such that the reference overnight rate in an OIS exactly matches the average cost of unsecured overnight funding of EURIBOR (the EUR equivalent of LIBOR) panel banks, providing a check of this assumption. And, third, the main shocks to the interbank money market in the second half of the sample period emanated from the Eurozone with its sovereign debt crisis. Indeed, we find that EUR interbank risk is generally higher than USD interbank risk in the second half of the sample period, while the opposite is true in the first half. Nevertheless, results on the decomposition of interbank risk, the drivers of the residual factor, and the risk compensation in the swap market are quite similar to the USD market.

Our analysis has several practical applications. First, the framework could be a valuable tool for central banks and regulatory authorities, as it provides market expectations about future stress in the interbank money market. In addition, the decomposition into default and non-default (liquidity) components can help guide appropriate policy responses (recapitalization of banks, termination/introduction of central bank lending facilities, etc). For instance, our analysis suggests that policy responses in the aftermath of the Lehman Brothers default were effective at resolving liquidity issues in the interbank market, but have been less effective at addressing default risk, particularly at longer horizons. ${ }^{8}$

Second, the model has implications for pricing, hedging, and risk-management in the interest rate swap market. Since the onset of the credit crisis, market participants have been exposed to significant basis risk: swap cash flows are indexed to LIBOR but, because of collateral agreements, are discounted using rates inferred from the OIS market. Furthermore, swap portfolios at most financial institutions are composed of swap contracts indexed to LIBOR rates of various maturities creating another layer of basis risks. Our model provides a useful framework for managing overall interest rate risk and these basis risks in an integrated way.

Our paper is related to Collin-Dufresne and Solnik (2001), who study the term structure of spreads between yields on corporate bonds issued by LIBOR banks and IRS rates. In their

\footnotetext{
${ }^{8}$ From a regulatory standpoint, the framework could also prove helpful in determining the right discount curve for the valuation of long-term insurance liabilities, where discount factors are typically allowed to include a liquidity component but not a default risk component.
} 
model, a spread arises because bond yields reflect the possibility of deterioration in the credit quality of current LIBOR banks relative to that of future LIBOR banks. As described above, in our model a similar mechanism underlies the default component of LIBOR-OIS and IRSOIS spreads. Our paper is also related to Liu, Longstaff, and Mandell (2006), Johannes and Sundaresan (2007), and Feldhutter and Lando (2008), who study the term structure of spreads between IRS rates and Treasury yields. ${ }^{9}$ Our paper has a different focus than these papers and also has the methodological advantage of not using bonds, the prices of which were heavily influenced by liquidity issues during the financial crisis. By only considering swap contracts, we expect liquidity to be less of an issue and to be more uniform across instruments leading to a clean decomposition of the term structure of interbank risk.

A number of papers have analyzed the 3M LIBOR-OIS spread and attempted to decompose it into default and liquidity components. These papers include Schwartz (2010), Taylor and Williams (2009), McAndrews, Sarkar, and Wang (2008), Michaud and Upper (2008), and Eisenschmidt and Tapking (2009). They all study the early phase of the financial crisis before the collapse of Lehman Brothers and find, with the exception of Taylor and Williams (2009), that liquidity was a key driver of interbank risk during this period. We find a similar result for the short end of the term structure of interbank risk. However, at the longer end of the term structure of interbank risk, default risk appears to have been the dominant driver even during the early phase of the financial crisis, underscoring the importance of taking the entire term structure into account when analyzing interbank risk. ${ }^{10}$

Several papers including Bianchetti (2009), Fujii, Shimada, and Takahashi (2009), Henrard (2009), and Mercurio $(2009,2010)$ have developed pricing models for interest rate derivatives that take the stochastic IRS-OIS spread into account. These are highly reduced-form models in that swap spreads indexed to different LIBOR rates are modeled independently of each other and also not decomposed into different components. In contrast, we provide a unified model of all such spreads, making it possible to aggregate the risks of large swap portfolios

\footnotetext{
${ }^{9}$ Feldhutter and Lando (2008) allow for a non-default component in the spread between LIBOR and the (unobservable) risk-free rate, which they argue is related to hedging flows in the IRS market. One shortcoming of their model, when applied to crisis data, is that it does not allow for credit quality deterioration in the valuation of LIBOR loans.

${ }^{10}$ Smith (2010) studies LIBOR-OIS spreads of maturities up to $12 \mathrm{M}$ within a dynamic term structure model and attributes the most of the variation in spreads to variation in risk-premia. A somewhat problematic aspect of her analysis is that the default component of LIBOR-OIS spreads is identified by the spread between LIBOR and repo rates, which clearly contains a significant liquidity component during much of the period.
} 
and analyze their underlying determinants.

The rest of the paper is organized as follows: Section 2 describes the market instruments. Section 3 describes the model of the term structure of interbank risk. Section 4 discusses the data and the estimation approach. Section 5 presents the results. Section 6 considers a variety of robustness checks. Section 7 concludes, and several appendices, including an online appendix, contain additional material.

\section{Market instruments}

We describe the market instruments that we use in the paper. We first consider the basic reference rates and then a variety of swap contracts that are indexed to these reference rates.

\subsection{Reference rates}

A large number of fixed income contracts are tied to an interbank offered rate. The main reference rate in the USD-denominated fixed income market is the USD London Interbank Offered Rate (LIBOR), while in the EUR-denominated fixed income market it is the European Interbank Offered Rate (EURIBOR). ${ }^{11}$ Both LIBOR and EURIBOR are trimmed averages of rates submitted by sets of banks. In the case of LIBOR, each contributor bank bases its submission on the question at what rate could you borrow funds, were you to do so by asking for and then accepting interbank offers in a reasonable market size. In the case of EURIBOR, the wording is slightly different and each contributor bank submits the rates at which euro interbank term deposits are being offered within the euro zone by one prime bank to another. Therefore, LIBOR is an average of the rates at which banks believe they can obtain unsecured funding, while EURIBOR is an average of the rates at which banks believe a prime bank can obtain unsecured funding. This subtle difference becomes important when quantifying the degree of default risk inherent in the two rates. Both rates are quoted for a range of terms, with $3 \mathrm{M}$ and $6 \mathrm{M}$ being the most important and most widely followed. In the following, we let $L(t, T)$ denote the $(T-t)$-maturity LIBOR or EURIBOR rate that fixes at time $t$.

For both LIBOR and EURIBOR, contributor banks are selected based on their credit quality and the scale of their market activities. During our sample period, the LIBOR panel

\footnotetext{
${ }^{11}$ LIBOR is managed by the British Bankers' Association, while EURIBOR is managed by the European Banking Federation. There also exists a EUR LIBOR, although this rate has not received the same benchmark status as EURIBOR.
} 
consisted of 16 banks, while the EURIBOR panel was significantly larger and consisted of 42 banks. ${ }^{12}$ An important feature of both panels is that they are reviewed and revised periodically. A bank that experiences a significant deterioration in its credit quality (and/or its market share) will be dropped from the panel and be replaced by a bank with superior credit quality.

An increasing number of fixed income contracts are tied to an index of overnight rates. In the USD market, the benchmark is the effective Federal Funds (FF) rate, which is a transactionweighted average of the rates on overnight unsecured loans of reserve balances held at the Federal Reserve that banks make to one another. In the EUR market, the benchmark is the Euro Overnight Index Average (EONIA) rate, computed as a transaction-weighted average of the rates on all overnight unsecured loans in the interbank money market initiated by EURIBOR panel banks. Therefore, in the EUR market, the benchmark overnight rate reflects the average cost of unsecured overnight funding of panel banks. We assume that the same holds for the USD market, although the set of banks from which the effective Federal Funds rate is computed does not exactly match the LIBOR panel. ${ }^{13}$

For the sake of convenience we will from now on use "LIBOR" as a generic term for an interbank offered rate, comprising both LIBOR and EURIBOR, whenever there is no ambiguity.

\subsection{Pricing collateralized contracts}

Swap contracts between major financial institutions are virtually always collateralized to the extent that counterparty risk is negligible. ${ }^{14}$ In this section, we provide the generic pricing formula of collateralized cashflows that we will use below to price swap contracts. ${ }^{15}$ Consider a contract with a contractual nominal cashflow $X$ at maturity $T$. Its present value at $t<T$ is denoted by $V(t)$. We assume that the two parties in the contract agree on posting cashcollateral on a continuous marking-to-market basis. We also assume that, at any time $t<T$,

\footnotetext{
${ }^{12}$ After the end of our sample period, the USD LIBOR panel was expanded to 20 banks and the EURIBOR panel was expanded to 44 banks.

${ }^{13}$ Participants in the Federal Funds market are those with accounts at Federal Reserve Banks, which include US depository institutions, US branches of foreign banks, and government-sponsored enterprises.

${ }^{14}$ Even in the absence of collateralization, counterparty risk usually has only a very small effect on the valuation of swap contracts; see, e.g., Duffie and Huang (1996). This led to the approach to interest rate swap pricing in Duffie and Singleton (1997).

${ }^{15}$ Similar formulas have been derived in various contexts by Johannes and Sundaresan (2007), Fujii, Shimada, and Takahashi (2009), and Piterbarg (2010).
} 
the posted amount of collateral equals $100 \%$ of the contract's present value $V(t)$. The receiver of the collateral can invest it at the risk-free rate $r(t)$ and has to pay an agreed rate $r_{c}(t)$ to the poster of collateral. The present value thus satisfies the following integral equation

$$
V(t)=E_{t}^{Q}\left[e^{-\int_{t}^{T} r(s) d s} X+\int_{t}^{T} e^{-\int_{t}^{u} r(s) d s}\left(r(u)-r_{c}(u)\right) V(u) d u\right],
$$

where $E_{t}^{Q} \equiv E^{Q}\left[\cdot \mid \mathcal{F}_{t}\right]$ denotes conditional expectation under the risk-neutral measure $Q .{ }^{16}$ It is shown in Appendix A that this implies the pricing formula

$$
V(t)=E_{t}^{Q}\left[e^{-\int_{t}^{T} r_{c}(s) d s} X\right] .
$$

For $X=1$, we obtain the price of a collateralized zero-coupon bond

$$
P_{c}(t, T)=E_{t}^{Q}\left[e^{-\int_{t}^{T} r_{c}(s) d s}\right] .
$$

In the sequel, we assume that the collateral rate $r_{c}(t)$ is equal to an instantaneous proxy $L(t, t)$ of the overnight rate, which we define as

$$
r_{c}(t)=L(t, t)=\lim _{T \rightarrow t} L(t, T)
$$

In reality, best practice among major financial institutions is daily mark-to-market and adjustment of collateral. Furthermore, cash collateral is the most popular form of collateral, since it is free from the issues associated with rehypothecation and allows for faster settlement times. Finally, FF and EONIA are typically the contractual interest rates earned by cash collateral in the USD and EUR markets, respectively. The assumptions we make above, therefore, closely approximate current market reality. ${ }^{17}$

\section{$2.3 \quad$ Interest rate swaps (IRS)}

In a regular interest rate swap (IRS), counterparties exchange a stream of fixed-rate payments for a stream of floating-rate payments indexed to LIBOR of a particular maturity. More specifically, consider two discrete tenor structures

$$
t=t_{0}<t_{1}<\cdots<t_{N}=T
$$

\footnotetext{
${ }^{16}$ Throughout, we assume a filtered probability space $\left(\Omega, \mathcal{F}, \mathcal{F}_{t}, Q\right)$, where $Q$ is a risk-neutral pricing measure.

${ }^{17}$ ISDA (2010) is a detailed survey of current market practice. Further evidence for the pricing formula given in this section is provided by Whittall (2010), who reports that the main clearing-house of interbank swap contracts now uses discount factors extracted from the OIS term structure to discount collateralized swap cashflows.
} 
and

$$
t=T_{0}<T_{1}<\cdots<T_{n}=T,
$$

and let $\delta=t_{i}-t_{i-1}$ and $\Delta=T_{i}-T_{i-1}$ denote the lengths between tenor dates, with $\delta<\Delta .{ }^{18}$ At every time $t_{i}, i=1, \ldots, N$, one party pays $\delta L\left(t_{i-1}, t_{i}\right)$, while at every time $T_{i}, i=1, \ldots, n$, the other party pays $\Delta K$, where $K$ denotes the fixed rate on the swap. The swap rate, $\operatorname{IR} S_{\delta, \Delta}(t, T)$, is the value of $K$ that makes the IRS value equal to zero at inception and is given by

$$
\operatorname{IR} S_{\delta, \Delta}(t, T)=\frac{\sum_{i=1}^{N} E_{t}^{Q}\left[e^{-\int_{t}^{t_{i}} r_{c}(s) d s} \delta L\left(t_{i-1}, t_{i}\right)\right]}{\sum_{i=1}^{n} \Delta P_{c}\left(t, T_{i}\right)} .
$$

In the USD market, the benchmark IRS pays 3M LIBOR floating vs. $6 \mathrm{M}$ fixed, while in the EUR market, the benchmark IRS pays 6M EURIBOR floating vs. $1 Y$ fixed. Rates on IRS indexed to LIBOR of other maturities are obtained via basis swaps as discussed below.

\subsection{Basis swaps (BS)}

In a basis swap (BS), counterparties exchange two streams of floating-rate payments indexed to LIBOR of different maturities, plus a stream of fixed payments. The quotation convention for basis swaps differs across brokers, across markets, and may also have changed over time. ${ }^{19}$ However, as demonstrated in the online appendix, the differences between the conventions are negligible. Consider a basis swap in which one party pays the $\delta_{1}$-maturity LIBOR while the other party pays the $\delta_{2}$-maturity LIBOR with $\delta_{1}<\delta_{2}$. We use the quotation convention in which the basis swap rate, $B S_{\delta_{1}, \delta_{2}}(t, T)$, is given as the difference between the fixed rates on two IRS indexed to $\delta_{2^{-}}$and $\delta_{1}$-maturity LIBOR, respectively. That is,

$$
B S_{\delta_{1}, \delta_{2}}(t, T)=\operatorname{IR} S_{\delta_{2}, \Delta}(t, T)-I R S_{\delta_{1}, \Delta}(t, T) .
$$

This convention has the advantage that rates on non-benchmark IRS are very easily obtained via basis swaps.

\subsection{Overnight indexed swaps (OIS)}

In an overnight indexed swap (OIS), counterparties exchange a stream of fixed-rate payments for a stream of floating-rate payments indexed to a compounded overnight rate (FF or EONIA).

\footnotetext{
${ }^{18}$ In practice, the length between dates will vary slightly depending on the day-count convention. To simplify notation, we suppress this dependence.

${ }^{19}$ We thank Fabio Mercurio for discussions about basis swap market conventions.
} 
More specifically, consider the tenor structure (6) with $\Delta=T_{i}-T_{i-1} \cdot{ }^{20}$ At every time $T_{i}$, $i=1, \ldots, N$, one party pays $\Delta K$, while the other party pays $\Delta \bar{L}\left(T_{i-1}, T_{i}\right)$, where $\bar{L}\left(T_{i-1}, T_{i}\right)$ is the compounded overnight rate for the period $\left[T_{i-1}, T_{i}\right]$. This rate is given by

$$
\bar{L}\left(T_{i-1}, T_{i}\right)=\frac{1}{\Delta}\left(\prod_{j=1}^{K_{i}}\left(1+\left(t_{j}-t_{j-1}\right) L\left(t_{j-1}, t_{j}\right)\right)-1\right)
$$

where $T_{i-1}=t_{0}<t_{1}<\cdots<t_{K_{i}}=T_{i}$ denotes the partition of the period $\left[T_{i-1}, T_{i}\right]$ into $K_{i}$ business days, and $L\left(t_{j-1}, t_{j}\right)$ denotes the respective overnight rate. As in Andersen and Piterbarg (2010, Section 5.5), we approximate simple by continuous compounding and the overnight rate by the instantaneous rate $L(t, t)$ given in (4), in which case $\bar{L}\left(T_{i-1}, T_{i}\right)$ becomes

$$
\bar{L}\left(T_{i-1}, T_{i}\right)=\frac{1}{\Delta}\left(e^{\int_{T_{i-1}}^{T_{i}} r_{c}(s) d s}-1\right) .
$$

The OIS rate is the value of $K$ that makes the OIS value equal to zero at inception and is given by

$$
O I S(t, T)=\frac{\sum_{i=1}^{n} E_{t}^{Q}\left[e^{-\int_{t}^{T_{i}} r_{c}(s) d s} \Delta \bar{L}\left(T_{i-1}, T_{i}\right)\right]}{\sum_{i=1}^{n} \Delta P_{c}\left(t, T_{i}\right)}=\frac{1-P_{c}\left(t, T_{n}\right)}{\sum_{i=1}^{n} \Delta P_{c}\left(t, T_{i}\right)} .
$$

In both the USD and EUR markets, OIS payments occur at a $1 Y$ frequency, i.e. $\Delta=1$. For OISs with maturities less than one year, there is only one payment at maturity.

\subsection{The IRS-OIS spread}

Combining (7) and (11), a few calculations yield

$$
\operatorname{IRS}_{\delta, \Delta}(t, T)-O I S(t, T)=\frac{\sum_{i=1}^{N} E_{t}^{Q}\left[e^{-\int_{t}^{t_{i}} r_{c}(s) d s} \delta\left(L\left(t_{i-1}, t_{i}\right)-O I S\left(t_{i-1}, t_{i}\right)\right)\right]}{\sum_{i=1}^{n} \Delta P_{c}\left(t, T_{i}\right)} .
$$

This equation shows that the spread between the rates on, say, a 5Y IRS indexed to $\delta$ maturity LIBOR and a $5 \mathrm{Y}$ OIS reflects (risk-neutral) expectations about future $\delta$-maturity LIBOR-OIS spreads during the next 5 years. ${ }^{21}$ To the extent that the LIBOR-OIS spread

\footnotetext{
${ }^{20}$ In contrast to an IRS, an OIS typically has fixed-rate payments and floating-rate payments occurring at the same frequency.

${ }^{21}$ Note that (12) only holds true if the fixed payments are made with the same frequency in the two swaps, which is the case in the EUR market but not in the USD market. For the more general case, suppose that the payments in the OIS are made on the tenor structure $t=T_{0}^{\prime}<T_{1}^{\prime}<\cdots<T_{n^{\prime}}^{\prime}=T$, with $\Delta^{\prime}=T_{i}^{\prime}-T_{i-1}^{\prime}$. Then one can show that (12) holds with $O I S\left(t_{i-1}, t_{i}\right)$ replaced by $w(t) O I S\left(t_{i-1}, t_{i}\right)$, where $w(t)=\frac{\sum_{i=1}^{n} \Delta P_{c}\left(t, T_{i}\right)}{\sum_{i=1}^{n^{\prime}} \Delta^{\prime} P_{c}\left(t, T_{i}^{\prime}\right)}$. In the USD market, where $\Delta=1 / 2$ and $\Delta^{\prime}=1, w(t)$ is always very close to one and (12) holds up to a very small approximation error.
} 
measures short-term interbank risk, the IRS-OIS spread reflects expectations about future short-term interbank risks - more specifically, about short-term interbank risks among the banks that constitute the LIBOR panel at future tenor dates, which may vary due to the periodic updating of the LIBOR panel. Consequently, we refer to the term structure of IRSOIS spreads as the term structure of interbank risk.

\subsection{Credit default swaps (CDS)}

In a credit default swap (CDS), counterparties exchange a stream of coupon payments for a single default protection payment in the event of default by a reference entity. As such, the swap comprises a premium leg (the coupon stream) and a protection leg (the contingent default protection payment). More specifically, consider the tenor structure (5) and let $\tau$ denote the default time of the reference entity. ${ }^{22}$ The present value of the premium leg with coupon rate $C$ is given by

$$
V_{\text {prem }}(t, T)=C I_{1}(t, T)+C I_{2}(t, T)
$$

where $C I_{1}(t, T)$ with

$$
I_{1}(t, T)=E_{t}^{Q}\left[\sum_{i=1}^{N} e^{-\int_{t}^{t_{i}} r_{c}(s) d s}\left(t_{i}-t_{i-1}\right) 1_{\left\{t_{i}<\tau\right\}}\right]
$$

is the value of the coupon payments prior to default time $\tau$, and $C I_{2}(t, T)$ with

$$
I_{2}(t, T)=E_{t}^{Q}\left[\sum_{i=1}^{N} e^{-\int_{t}^{\tau} r_{c}(s) d s}\left(\tau-t_{i-1}\right) 1_{\left\{t_{i-1}<\tau \leq t_{i}\right\}}\right]
$$

is the accrued coupon payment at default time $\tau$. The present value of the protection leg is

$$
V_{\operatorname{prot}}(t, T)=E_{t}^{Q}\left[e^{-\int_{t}^{\tau} r_{c}(s) d s}(1-R(\tau)) 1_{\{\tau \leq T\}}\right]
$$

where $R(\tau)$ denotes the recovery rate at default time $\tau$. The CDS spread, $C D S(t, T)$, is the value of $C$ that makes the premium and protection leg equal in value at inception and is given

\footnotetext{
${ }^{22} \mathrm{CDS}$ contracts are traded with maturity dates falling on one of four roll dates, March 20, June 20, September 20, or December 20. At initiation, therefore, the actual time to maturity of a CDS contract will be close to, but rarely the same as, the specified time to maturity. Coupon payments are made on a quarterly basis coinciding with the CDS roll dates.
} 
by $^{23}$

$$
C D S(t, T)=\frac{V_{\text {prot }}(t, T)}{I_{1}(t, T)+I_{2}(t, T)}
$$

\section{Modeling the term structure of interbank risk}

We describe our model of the term structure of interbank risk. We first consider the general framework and then specialize to a tractable model with analytical pricing formulas.

\subsection{The general framework}

Rather than modeling the funding costs of individual panel banks, we consider an average bank which represents the panel at a given point in time. More specifically, we assume the extended doubly stochastic framework provided in Appendix B below, where for any $t_{0} \geq 0$, the default time of an average bank within the $t_{0}$-panel is modeled by some random time $\tau\left(t_{0}\right)>t_{0}$. This default time admits a nonnegative intensity process $\lambda\left(t_{0}, t\right)$, for $t>t_{0}$, with initial value $\lambda\left(t_{0}, t_{0}\right)=\Lambda\left(t_{0}\right)$. In other words, at a given point in time $t>t_{0}, \Lambda(t)$ is the average default intensity of the current $t$-panel, while $\lambda\left(t_{0}, t\right)$ is the default intensity of an average bank within the initial $t_{0}$-panel.

In view of the doubly stochastic property (39), the time $t_{0}$-value of an unsecured loan with notional 1 to an average bank within the $t_{0}$-panel over period $\left[t_{0}, T\right]$ equals

$$
B\left(t_{0}, T\right)=E_{t_{0}}^{Q}\left[e^{-\int_{t_{0}}^{T} r(s) d s} 1_{\left\{\tau\left(t_{0}\right)>T\right\}}\right]=E_{t_{0}}^{Q}\left[e^{-\int_{t_{0}}^{T}\left(r(s)+\lambda\left(t_{0}, s\right)\right) d s}\right] .
$$

Note that here we assume zero recovery of interbank loans, which is necessary to keep the subsequent affine transform analysis tractable. ${ }^{24}$ Absent market frictions, the $\left(T-t_{0}\right)$-maturity LIBOR rate $L\left(t_{0}, T\right)$ satisfies $1+\left(T-t_{0}\right) L\left(t_{0}, T\right)=1 / B\left(t_{0}, T\right)$.

In practice, LIBOR may be affected by factors not directly related to default risk. For instance, banks may refrain from lending long-term for precautionary reasons as in the models of Allen, Carletti, and Gale (2009) and Acharya and Skeie (2010), or for speculative reasons as in

\footnotetext{
${ }^{23}$ While these "par spreads" are quoted in the market, CDS contracts have been executed since 2009 with a standardized coupon and an upfront payment to compensate for the difference between the par spread and the coupon. However, our CDS database consists of par spreads throughout the sample period.

${ }^{24}$ Alternatively, we could follow Duffie and Singleton (1999) and let $\lambda\left(t_{0}, s\right)=h\left(t_{0}, s\right) l\left(t_{0}, s\right)$ be the product of a default intensity process, $h\left(t_{0}, s\right)$, and a fractional default loss process, $l\left(t_{0}, s\right)$. That is, $l\left(t_{0}, s\right) \in[0,1]$ defines the fraction of market value of the loan that is lost upon default.
} 
the models of Acharya, Gromb, and Yorulmazer (2007), Acharya, Shin, and Yorulmazer (2010), and Diamond and Rajan (2010). Either way, the volume of longer term interbank loans decreases and the rates on such loans increase beyond the levels justified by default risk. We allow for a non-default component in LIBOR by setting

$$
L\left(t_{0}, T\right)=\frac{1}{T-t_{0}}\left(\frac{1}{B\left(t_{0}, T\right)}-1\right) \Xi\left(t_{0}, T\right),
$$

where $\Xi\left(t_{0}, T\right)$ is a multiplicative residual term that satisfies

$$
\lim _{T \rightarrow t_{0}} \Xi\left(t_{0}, T\right)=1 \text {. }
$$

It follows from (4) that the collateral rate $r_{c}\left(t_{0}\right)$ becomes

$$
r_{c}\left(t_{0}\right)=\lim _{T \rightarrow t_{0}} \frac{1}{T-t_{0}}\left(\frac{1}{B\left(t_{0}, T\right)}-1\right) \Xi\left(t_{0}, T\right)=-\left.\frac{d}{d T} B\left(t_{0}, T\right)\right|_{T=t_{0}}=r\left(t_{0}\right)+\Lambda\left(t_{0}\right) .
$$

Combining (11) (in the case of a single payment) and (17), we get the following expression for the LIBOR-OIS spread

$$
L\left(t_{0}, T\right)-O I S\left(t_{0}, T\right)=\frac{1}{T-t_{0}}\left(\left[\frac{1}{B\left(t_{0}, T\right)}-\frac{1}{P_{c}\left(t_{0}, T\right)}\right]+\left[\left(\frac{1}{B\left(t_{0}, T\right)}-1\right)\left(\Xi\left(t_{0}, T\right)-1\right)\right]\right) .
$$

The first bracketed term in (19) is the default component. The periodic updating of the LIBOR panel implies that $\lambda\left(t_{0}, t\right) \geq \Lambda(t)$, for $t>t_{0}$. From (16) and (3) in conjunction with (18) it follows that $B\left(t_{0}, T\right)>P_{c}\left(t_{0}, T\right)$, which implies that the default component is positive. The second bracketed term in (19) is the non-default component, which is positive provided that $\Xi\left(t_{0}, T\right)>1$.

For the analysis, we also need expressions for the CDS spreads of an average bank within the $t_{0}$-panel. The factors $I_{1}\left(t_{0}, T\right)$ and $I_{2}\left(t_{0}, T\right)$ in the present value of the premium leg given in (13) and (14) become

$$
I_{1}\left(t_{0}, T\right)=\sum_{i=1}^{N}\left(t_{i}-t_{i-1}\right) E_{t_{0}}^{Q}\left[e^{-\int_{t_{0}}^{t_{i}} r_{c}(s) d s} 1_{\left\{t_{i}<\tau\left(t_{0}\right)\right\}}\right]=\sum_{i=1}^{N}\left(t_{i}-t_{i-1}\right) E_{t_{0}}^{Q}\left[e^{-\int_{t_{0}}^{t_{i}}\left(r_{c}(s)+\lambda\left(t_{0}, s\right)\right) d s}\right]
$$

$\operatorname{and}^{25}$

$$
\begin{aligned}
I_{2}\left(t_{0}, T\right) & =\sum_{i=1}^{N} E_{t_{0}}^{Q}\left[e^{-\int_{t_{0}}^{\tau\left(t_{0}\right)} r_{c}(s) d s}\left(\tau\left(t_{0}\right)-t_{i-1}\right) 1_{\left\{t_{i-1}<\tau\left(t_{0}\right) \leq t_{i}\right\}}\right] \\
& =\sum_{i=1}^{N} \int_{t_{i-1}}^{t_{i}}\left(u-t_{i-1}\right) E_{t_{0}}^{Q}\left[e^{-\int_{t_{0}}^{u}\left(r_{c}(s)+\lambda\left(t_{0}, s\right)\right) d s} \lambda\left(t_{0}, u\right)\right] d u .
\end{aligned}
$$

\footnotetext{
${ }^{25}$ Here we use the fact that, using the terminology of Appendix B below, $e^{-\int_{t_{0}}^{u} \lambda\left(t_{0}, s\right) d s} \lambda\left(t_{0}, u\right)$ is the $\mathcal{F}_{\infty} \vee \mathcal{H}_{t_{0}}$ conditional density function of $\tau\left(t_{0}\right)$, see e.g. Filipović (2009, Section 12.3).
} 
In line with the assumption of zero recovery of interbank loans in the derivation of (16), we shall assume zero recovery for the CDS protection leg. Its present value (15) thus becomes

$$
V_{\text {prot }}\left(t_{0}, T\right)=E_{t_{0}}^{Q}\left[e^{-\int_{t_{0}}^{\tau\left(t_{0}\right)} r_{c}(s) d s} 1_{\left\{\tau\left(t_{0}\right) \leq T\right\}}\right]=\int_{t_{0}}^{T} E_{t_{0}}^{Q}\left[e^{-\int_{t_{0}}^{u}\left(r_{c}(s)+\lambda\left(t_{0}, s\right)\right) d s} \lambda\left(t_{0}, u\right)\right] d u .
$$

\subsection{An affine factor model}

We now introduce an affine factor model of $r(t)$, the intensities $\Lambda(t)$ and $\lambda\left(t_{0}, t\right)$, and the residual $\Xi\left(t_{0}, T\right)$. We assume that the risk-free short rate, $r(t)$, is driven by a two-factor Gaussian process $^{26}$

$$
\begin{aligned}
& d r(t)=\kappa_{r}(\gamma(t)-r(t)) d t+\sigma_{r} d W_{r}(t) \\
& d \gamma(t)=\kappa_{\gamma}\left(\theta_{\gamma}-\gamma(t)\right) d t+\sigma_{\gamma}\left(\rho d W_{r}(t)+\sqrt{1-\rho^{2}} d W_{\gamma}(t)\right),
\end{aligned}
$$

where $\gamma(t)$ is the stochastic mean-reversion level of $r(t)$, and $\rho$ is the correlation between innovations to $r(t)$ and $\gamma(t)$.

We have investigated several specifications for the average default intensity of the periodically refreshed panel, $\Lambda(t)$. In the interest of parsimony, we assume that $\Lambda(t)$ is constant

$$
\Lambda(t) \equiv \Lambda
$$

In Section 6, we analyze a setting, where $\Lambda(t)$ is stochastic. This adds complexity to the model without materially affecting the results.

The default intensity of an average bank within the $t_{0}$-panel, $\lambda\left(t_{0}, t\right)$, is modeled by

$$
\lambda\left(t_{0}, t\right)=\Lambda+\int_{t_{0}}^{t} \kappa_{\lambda}\left(\Lambda-\lambda\left(t_{0}, s\right)\right) d s+\sum_{j=N\left(t_{0}\right)+1}^{N(t)} Z_{\lambda, j},
$$

where $N(t)$ is a simple counting process with jump intensity $\nu(t)$ and $Z_{\lambda, 1}, Z_{\lambda, 2}, \ldots$ are i.i.d. exponential jump sizes with mean $\frac{1}{\zeta_{\lambda}}$. That is, we assume that deterioration in the credit quality of an average bank within the $t_{0}$-panel relative to the average credit quality of the periodically refreshed panel occurs according to a jump process. The first jump time of $\lambda\left(t_{0}, t\right)$ is interpreted as the time, when the bank is dropped from the panel. Between jumps, we allow for $\lambda\left(t_{0}, t\right)$ to mean-revert towards $\Lambda .^{27}$

\footnotetext{
${ }^{26}$ The model is equally tractable with $r(t)$ being driven by a two-factor square-root process. While this may seem more appropriate given the low interest rate environment during much of the sample period, we found that the fit to the OIS term structure is slightly worse with this specification. Nevertheless, the decomposition of the term structure of interbank risk is almost identical for two specifications.

${ }^{27}$ In Section 6, we explore an alternative specification, where deterioration in credit quality is permanent.
} 
The intensity of credit quality deterioration, $\nu(t)$, is stochastic and evolves according to either a one-factor square-root process

$$
d \nu(t)=\kappa_{\nu}\left(\theta_{\nu}-\nu(t)\right) d t+\sigma_{\nu} \sqrt{\nu(t)} d W_{\nu}(t)
$$

or a two-factor square-root process

$$
\begin{aligned}
& d \nu(t)=\kappa_{\nu}(\mu(t)-\nu(t)) d t+\sigma_{\nu} \sqrt{\nu(t)} d W_{\nu}(t) \\
& d \mu(t)=\kappa_{\mu}\left(\theta_{\mu}-\mu(t)\right) d t+\sigma_{\mu} \sqrt{\mu(t)} d W_{\mu}(t),
\end{aligned}
$$

where $\mu(t)$ is the stochastic mean-reversion level of $\nu(t)$.

Finally, the multiplicative residual term, $\Xi\left(t_{0}, T\right)$, is modeled by ${ }^{28}$

$$
\frac{1}{\Xi\left(t_{0}, T\right)}=E_{t_{0}}^{Q}\left[e^{-\int_{t_{0}}^{T} \xi(s) d s}\right]
$$

where $\xi(t)$ evolves according to either a one-factor square-root process

$$
d \xi(t)=\kappa_{\xi}\left(\theta_{\xi}-\xi(t)\right) d t+\sigma_{\xi} \sqrt{\xi(t)} d W_{\xi}(t)
$$

or a two-factor square-root process

$$
\begin{gathered}
d \xi(t)=\kappa_{\xi}(\epsilon(t)-\xi(t)) d t+\sigma_{\xi} \sqrt{\xi(t)} d W_{\xi}(t) \\
d \epsilon(t)=\kappa_{\epsilon}\left(\theta_{\epsilon}-\epsilon(t)\right) d t+\sigma_{\epsilon} \sqrt{\epsilon(t)} d W_{\epsilon}(t)
\end{gathered}
$$

where $\epsilon(t)$ is the stochastic mean-reversion level of $\xi(t)$.

In the following, we will use the notation $\mathbb{A}(X, Y, Z)$ to denote a specification where $r(t)$, $\nu(t)$, and $\xi(t)$ are driven by $X, Y$, and $Z$ factors, respectively. We analyze three progressively more complex model specifications: $\mathbb{A}(2,1,1)$, where the state vector is given by $(23),(25)$, and (28), $\mathbb{A}(2,2,1)$, where the state vector is given by (23), (26), and (28), and $\mathbb{A}(2,2,2)$, where the state vector is given by (23), (26), and (29). All specifications have analytical pricing formulas for LIBOR, OIS, IRS, and CDS. These formulas are given in Appendix C, which also contains sufficient admissability conditions on the parameter values (Lemma C.4).

For the empirical part, we also need the dynamics of the state vector under the objective probability measure $P \sim Q$. Given our relatively short sample period, we assume a parsimonious market price of risk process

$$
\Gamma(t)=\left(\Gamma_{r}, \Gamma_{\gamma}, \Gamma_{\nu} \sqrt{\nu(t)}, \Gamma_{\mu} \sqrt{\mu(t)}, \Gamma_{\xi} \sqrt{\xi(t)}, \Gamma_{\epsilon} \sqrt{\epsilon(t)}\right)^{\top}
$$

\footnotetext{
${ }^{28}$ We specify $\Xi\left(t_{0}, T\right)$ to be non-decreasing in $T$. This is consistent with the economic fact that in the absence of negative rates the non-annualized LIBOR, $\left(T-t_{0}\right) L\left(t_{0}, T\right)$, which in our framework (17) factorizes as $\left(T-t_{0}\right) L\left(t_{0}, T\right)=\left(\frac{1}{B\left(t_{0}, T\right)}-1\right) \Xi\left(t_{0}, T\right)$, is non-decreasing in $T$.
} 
such that $d W(t)-\Gamma(t) d t$ becomes a standard Brownian motion under $P$ with Radon-Nikodym density process $^{29}$

$$
\left.\frac{d P}{d Q}\right|_{\mathcal{F}_{t}}=\exp \left(\int_{0}^{t} \Gamma(s)^{\top} d W(s)-\frac{1}{2} \int_{0}^{t}\|\Gamma(s)\|^{2} d s\right) .
$$

\section{Data and estimation}

We estimate the model on a panel data set that covers the period starting with the onset of the credit crisis on August 09, 2007 and ending on January 12, 2011. We do not include the pre-crisis period, given that a regime switch in the perception of interbank risk appear to have occurred at the onset of the crisis, see Figure 1.

\subsection{Interest rate data}

The interest rate data is from Bloomberg. We collect daily OIS rates with maturities $3 \mathrm{M}, 6 \mathrm{M}$, $1 \mathrm{Y}, 2 \mathrm{Y}, 3 \mathrm{Y}, 4 \mathrm{Y}, 5 \mathrm{Y}, 7 \mathrm{Y}$, and $10 \mathrm{Y} .{ }^{30}$ We also collect daily IRS and BS rates with maturities of $1 \mathrm{Y}, 2 \mathrm{Y}, 3 \mathrm{Y}, 4 \mathrm{Y}, 5 \mathrm{Y}, 7 \mathrm{Y}$, and $10 \mathrm{Y}$ as well as $3 \mathrm{M}$ and $6 \mathrm{M}$ LIBOR and EURIBOR rates. The rates on OIS, IRS, and BS are composite quotes computed from quotes that Bloomberg collects from major banks and inter-dealer brokers.

In the USD market, the benchmark IRS is indexed to 3M LIBOR (with fixed-rate payments occurring at a $6 \mathrm{M}$ frequency), and the rate on an IRS indexed to $6 \mathrm{M}$ LIBOR is obtained via a BS as

$$
I R S_{6 M, 6 M}(t, T)=I R S_{3 M, 6 M}(t, T)+B S_{3 M, 6 M}(t, T) .
$$

Conversely, in the EUR market, the benchmark IRS is indexed to 6M EURIBOR (with fixedrate payments occurring at a $1 \mathrm{Y}$ frequency), and the rate on an IRS indexed to $3 \mathrm{M}$ EURIBOR is obtained via a BS as

$$
I R S_{3 M, 1 Y}(t, T)=I R S_{6 M, 1 Y}(t, T)-B S_{3 M, 6 M}(t, T) .
$$

In the paper, we focus on the spreads between rates on IRS and OIS with the same maturities. Therefore, for each currency and on each day in the sample, we have two spread

\footnotetext{
${ }^{29}$ We charge no explicit premium for the jump intensity and size risk of $\lambda\left(t_{0}, t\right)$ in $(24)$.

${ }^{30}$ In Bloomberg, there is no USD 7 Y OIS rate. Also, the time series for the USD 10Y OIS rate starts July $28,2008$.
} 
term structures given by

$$
S P R E A D_{\delta}(t, T)=I R S_{\delta, \Delta}(t, T)-O I S(t, T),
$$

for $\delta=3 \mathrm{M}$ or $\delta=6 \mathrm{M}$ and $\Delta=6 \mathrm{M}(1 \mathrm{Y})$ in the USD (EUR) market.

Table 1 shows summary statistics of the data. For a given maturity, interest rate spreads are always increasing in the tenor (the maturity of the LIBOR rate to which an IRS is indexed). This is consistent with the idea that a $6 \mathrm{M}$ LIBOR loan contains more default and liquidity risk than two consecutive 3M LIBOR loans. For a given tenor, the mean and volatility of spreads decrease with maturity. While the mean spreads are similar across the two markets, spread volatility tends to be higher in the USD market.

\subsection{CDS spread data}

The CDS data is from Markit, which is the leading provider of CDS quotes. Markit collects quotes from major market participants and constructs daily composite quotes. Since data supplied by Markit is widely used for marking-to-market CDS contracts, its quotes are closely watched by market participants. For each bank in the LIBOR and EURIBOR panels, we collect daily spread term structures for CDS contracts written on senior obligations. The term structures consist of $6 \mathrm{M}, 1 \mathrm{Y}, 2 \mathrm{Y}, 3 \mathrm{Y}, 4 \mathrm{Y}, 5 \mathrm{Y}, 7 \mathrm{Y}$, and $10 \mathrm{Y}$ maturities.

Tables 2 and 3 shows summary statistics for the CDS spreads of the constituents of the LIBOR and EURIBOR panels, respectively. ${ }^{31}$ The tables also show the currency of the CDS contracts $^{32}$, the size of the banks' balance sheets as reported in the 2009 annual reports, a measure of liquidity of the CDS contracts, and the date from which CDS data is available in the Markit database.

\footnotetext{
${ }^{31}$ During our sample period, there were no revisions to the LIBOR and EURIBOR panels. As mentioned in Section 2.1, the EURIBOR panel consisted of 42 banks. Three of the smaller panel banks - Bank of Ireland, Banque et Caisse d'Epargne de l'Etat, and Confederacion Espanola de Cajas de Ahorros - were not in the Markit database.

${ }^{32}$ For European-based banks, the CDS contracts in the database supplied by Markit are denominated in EUR and subject to the Modified-Restructuring (MR) clause. For US-based banks, the CDS contracts are denominated in USD and subject to the MR clause until Dec 31, 2008 and the No-Restructuring (XR) clause thereafter. Finally, for Japan-based banks, the CDS contracts are denominated in JPY until Dec 31, 2008 and USD thereafter, and are subject to the Complete-Restructuring (CR) clause. Even though the currency denomination differs across CDS contracts, the CDS spreads are expressed as a rate and are, therefore, free of units of account.
} 
Our measure of liquidity is the average daily trading volume in terms of notional, as reported by the Depository Trust and Clearing Corporation (DTCC), a global repository that records the details of virtually all CDS trades in the global market. The data covers the period from June 20, 2009 to March 19, 2011 (data was not available prior to this period) and only includes trading activity that involves a transfer of risk between market participants. Also, the data only covers the top 1000 reference entities (in terms of the notional of outstanding contracts) and some banks, particularly from the EURIBOR panel, are not covered (or only covered during parts of the period, in which case we also do not report numbers).

We see that the LIBOR panel mainly consists of very large banks with significant trading activity in their CDS contracts, although it also includes some medium-sized banks for which the CDS contracts are traded less actively. For the EURIBOR panel, there is a larger crosssectional dispersion of the size of the member banks and the trading activity in their CDS contracts, which is natural given that the panel consists of significantly more banks than the LIBOR panel.

\subsection{Measures of interbank default risk}

To measure interbank default risk, we initially assume that CDS spreads are pure measures of the default risk of the underlying entities. At each point in time, we construct a CDS spread term structure for an average bank within the panel as a composite of the CDS spread term structures for the individual panel banks.

The LIBOR panel As discussed in Section 2.1, LIBOR is a trimmed mean of the rates at which banks estimate they can obtain unsecured funding for a given term. Since the submitted rates depend on the banks' own default risks, LIBOR itself presumably reflects a trimmed mean of the default risks of the panel banks. Therefore, we measure the default risk of an average bank within the LIBOR panel by aggregating the CDS spreads of the individual LIBOR panel banks in the same way that LIBOR is computed from the submitted rates, namely by removing the top and bottom 25 percent of spreads and computing a simple average of the remaining spreads. The resulting default risk measure is denoted $C D S_{T r M e a n}$.

The EURIBOR panel As we also discussed in Section 2.1, EURIBOR is a trimmed mean of the rates at which banks estimate a prime bank (not necessarily themselves) can obtain unsecured funding for a given term. While the notion of a prime bank is ambiguous, we 
interpret it as a representative bank among the panel. Since the median rather than the mean seems to be the appropriate statistics in this case, it is plausible that the submitted rates reflect what each bank perceives is the median default risk in the panel. Being a trimmed mean of the submitted rates, EURIBOR itself then also reflects the median default risk in the panel. Therefore, we measure the default risk of an average bank within the EURIBOR panel by taking the median of the CDS spreads of the individual EURIBOR panel banks. We denote this default risk measure $C D S_{\text {Median }}$.

Correcting for possible liquidity effects The assumption that CDS spreads are pure measures of default risk is made in several papers, including Longstaff, Mithal, and Neis (2005), Blanco, Brennan, and Marsh (2005), Beber, Brandt, and Kavajecz (2009), and Ang and Longstaff (2011). However, a number of recent papers have found that CDS spreads may be affected by liquidity effects. For instance, Buhler and Trapp (2010) find that, on average, 95 percent of the observed mid CDS spread is due to default risk, while the remaining is due to liquidity risk and the correlation between default and liquidity risk. This implies that the premium due to liquidity is earned by the seller of default protection and that CDS spreads are upward-biased measures of default risk. Similar results are reached by Bongaerts, de Jong, and Driessen (2011) and others.

In the case of LIBOR, liquidity may be less of an issue since the panel mainly consists of banks with relatively liquid CDS contracts, and since we use a trimmed mean of the individual CDS spreads, which reduces the effect of idiosyncratic noise at the level of the individual spreads. In the case of EURIBOR, where there is larger cross-sectional dispersion in the liquidity of the banks' CDS contracts, and where we work with a median spread, liquidity issues may be more important.

For both panels, we consider two alternative measures of default risks that correct for possible liquidity effects. First, we measure default risk by 90 percent of the composite CDS spreads, corresponding to a situation where protection sellers earn a significant liquidity premium. Given the results in Buhler and Trapp (2010), this is likely to be a lower bound on the default component of CDS spreads. Second, we measure default risk by constructing the composite CDS spreads as described above, but only using data from those banks where the average daily notional of CDS transactions are larger than 50 million USD equivalent. ${ }^{33}$ In

\footnotetext{
${ }^{33}$ To put these numbers into perspective, we computed summary statistics for the trading activity among the top 1000 reference entities that were not sovereigns. On a quarterly basis, the median varies between 15.0 and
} 
each market, the two alternative default risk measures are denoted $C D S_{L I Q 1}$ and $C D S_{L I Q 2}$.

iTraxx Senior Financials index As an alternative to computing composite CDS spreads from the panel constituents, for the EUR market we also consider the iTraxx Senior Financials CDS index. This index is quoted directly in the market and tracks the spreads on CDS contracts written on senior obligations of 25 large European financial institutions. The index tends to be more liquid than the individual contracts, but clearly both its construction and the fact that some of the underlying institutions are not part of EURIBOR ${ }^{34}$ makes it an imperfect measure of the default risk inherent in EURIBOR. Also, it is only available for maturities of $5 \mathrm{Y}$ and $10 \mathrm{Y}$. Nevertheless, it serves as an interesting robustness check.

Summary statistics Table 1 shows summary statistics of the composite CDS spreads. On average, the level of CDS spreads increase with maturity, while CDS spread volatility decrease with maturity. In the USD market, we have, on average, $C D S_{L I Q 1}<C D S_{\text {TrMean }}<$ $C D S_{L I Q 2}$, while in the EUR market, we have, on average, $C D S_{L I Q 1}<C D S_{L I Q 2}<C D S_{\text {Median }}<$ $C D S_{\text {Traxx }}$. The magnitudes of $C D S_{\text {TrMean }}$ in the USD market and $C D S_{\text {Median }}$ in the EUR market are rather similar despite the EURIBOR panel being composed of significantly more banks than the LIBOR panel.

Our main sets of results will be based on the original default risk measures, while in Section 6 we investigate the sensitivity of the results to taking possible CDS liquidity effects into account.

\subsection{Maximum-likelihood estimation}

We estimate the specifications using maximum-likelihood in conjunction with Kalman filtering. Due to the non-linearities in the relation between observations and state variables, we apply the non-linear unscented Kalman filter, which is found by Christoffersen et al. (2009) to have very good finite-sample properties in the context of estimating dynamic term structure models with swap rates. Details on the estimation approach are provided in Appendix D.

In terms of identification, we face several issues. First, we show in the online appendix that

20.8 million USD, while the mean varies between 25.0 and 33.6 million USD. With a cutoff of 50 million USD, we are clearly focusing on the most liquid segment of the CDS market.

${ }^{34}$ For instance, for the iTraxx series 14, launched in September 2010, 14 of the 25 financial institutions were also members of the EURIBOR panel. 
it is very difficult to separately identify $\zeta_{\lambda}$ (with $\frac{1}{\zeta_{\lambda}}$ being the mean jump size in the default intensity) and the process for $\nu(t)$ (the intensity of credit quality deterioration). Rather, it is the mean rate of credit quality deterioration of an average panel bank, $\frac{1}{\zeta_{\lambda}} \nu(t)$, that matters for valuation. In the estimation, we fix $\zeta_{\lambda}$ at 10 , but the implied process for the mean rate of credit quality deterioration is invariant to the choice of $\zeta_{\lambda}$.

Second, in a preliminary analysis, we find that it is difficult to reliably estimate the default intensity of the periodically refreshed panel, $\Lambda$. Its value is not identified from the OIS term structure and, in the absence of very short-term CDS spreads, is also hard to pin down from the CDS term structure. From (4) and (18), we have that $\Lambda$ is the difference between the instantaneous proxy of the overnight unsecured interbank rate, $L(t, t)$, and the truly risk-free rate, $r(t)$. Therefore, one can get an idea about the magnitude of $\Lambda$ by examining the spread between short-term OIS rates and repo rates, which are virtually riskfree due to the practice of overcollateralization of repo loans; see, e.g., Longstaff (2000). The sample averages of the 1W OIS-repo spreads for Treasury, Agency, Agency MBS, and European general collateral are $13 \mathrm{bp}, 3 \mathrm{bp},-1 \mathrm{bp}$, and $0 \mathrm{bp}$, respectively. ${ }^{35,36}$ Plots of these spreads can be found in the online appendix. In the case of Treasury collateral, the spread spikes at the beginning of the crisis and around the Bear Stearns and Lehman Brothers episodes. However, movements in the spread likely reflect periodic scarcity of Treasury collateral, rather than variation in default risk, since the spikes are mostly due to downward spikes in the Treasury repo rate rather than upward spikes in the OIS rate. Also, the correlation between the OIS-Treasury repo spread and short-maturity $(6 \mathrm{M})$ bank CDS spreads is virtually zero. ${ }^{37}$ In the cases of Agency and Agency MBS collateral, the spreads are volatile in the first half of the sample period, but without systematic patterns around crisis events, while in the case of the EUR market, the

\footnotetext{
${ }^{35}$ For the EUR market, we use the Eurepo rate, which is a benchmark reflecting the rate on interbank borrowing secured by "the best collateral within the most actively traded European repo market."

${ }^{36}$ Similar results are obtained by examining the spreads between FF and overnight repo rates for Treasury, Agency, and Agency MBS general collateral as well as the spread between EONIA and the overnight Eurepo rate. The sample averages of these spreads are $15 \mathrm{bp}, 5 \mathrm{bp}, 2 \mathrm{bp}$, and $1 \mathrm{bp}$, respectively. Since overnight rates are highly volatile with predictable liquidity-driven jumps (see, e.g., Bartolini et al. (2011)), we believe the 1W OIS-repo spreads are more informative.

${ }^{37}$ In their analysis of the repo market, Hordahl and King (2008) also notes scarcity of Treasury collateral as the main factor driving repo spreads: "As the available supply of Treasury collateral dropped, those market participants willing to lend out Treasuries were able to borrow cash at increasingly cheap rates. At times, this effect pushed US GC repo rates down to levels only a few basis points above zero".
} 
spread is very stable throughout the sample period. Taken together, these results suggest that there is very little default risk in the market for overnight interbank deposits. We fix $\Lambda$ at 5 $\mathrm{bp}$, but reasonable variations in the value of $\Lambda$ do not change our results. In Section 6, we show that our results are robust to extending the model with a stochastic $\Lambda(t)$ identified via OIS-Treasury repo spreads.

Third, given the relatively short sample period, many of the market price of risk parameters are imprecisely estimated (in contrasts to the risk-neutral parameters, most of which are strongly identified with low standard errors). For each model specification, we obtain a more parsimonious risk premium structure by reestimating the model after setting to zero those market price of risk parameters for which the absolute $t$-statistics did not exceed one. ${ }^{38}$ The likelihood functions were virtually unaffected by this, so we henceforth study these constrained model specifications.

\section{Results}

\subsection{Maximum-likelihood estimates}

Table 4 displays parameter estimates and their asymptotic standard errors. ${ }^{39}$ The estimates are strikingly similar across the two markets and, therefore, we focus on the USD estimates.

In the $\mathbb{A}(2,2,1)$ and $\mathbb{A}(2,2,2)$ specifications, $\nu(t)$ is relatively volatile and displays fast meanreversion towards $\mu(t)$, which in turn is less volatile and has much slower mean-reversion. Hence, $\nu(t)$ captures transitory shocks to the intensity of credit quality deterioration, while $\mu(t)$ captures more persistent shocks. In the $\mathbb{A}(2,1,1)$ specification, the speed of mean-reversion and volatility lie between those of $\nu(t)$ and $\mu(t)$ in the more general specifications. Also, between jumps, the reversion of the default intensity towards $\Lambda$ occurs relatively fast. Although estimated with some uncertainty, the market prices of risk $\Gamma_{\nu}$ and $\Gamma_{\mu}$ are negative in all specifications. This implies that the long-run mean of credit quality deterioration is lower under the physical measure than under the risk-neutral measure, indicating that market participants require a premium for bearing exposure to variation in default risk. ${ }^{40}$ We return to this issue

\footnotetext{
${ }^{38}$ A similar approach is taken by Duffee (2002) and Dai and Singleton (2002) and others.

${ }^{39}$ It is straightforward to verify that for all the specifications, the parameter values satisfy the sufficient admissability conditions in Lemma C.4 in the Appendix.

${ }^{40}$ In the $\mathbb{A}(2,1,1)$ specification, the long-run mean under the physical measure is given by $\theta_{\nu} \kappa_{\nu} /\left(\kappa_{\nu}-\sigma_{\nu} \Gamma_{\nu}\right)$, while in the $\mathbb{A}(2,2,1)$ and $\mathbb{A}(2,2,2)$ specifications, it is given by $\theta_{\mu} \kappa_{\nu} \kappa_{\mu} /\left[\left(\kappa_{\nu}-\sigma_{\nu} \Gamma_{\nu}\right)\left(\kappa_{\mu}-\sigma_{\mu} \Gamma_{\mu}\right)\right]$.
} 
in Section 5.6.

In all specifications, the residual factor, $\xi(t)$, is very volatile, exhibits very fast meanreversion, and has a long-run mean of essentially zero. In the $\mathbb{A}(2,2,2)$ specifications, $\xi(t)$ is mean-reverting towards $\epsilon(t)$ which is less volatile and has slower mean-reversion. Hence, $\xi(t)$ captures transitory shocks to the non-default component, while $\epsilon(t)$ captures moderately persistent shocks. In none of the specifications, were we able to reliably estimate $\Gamma_{\xi}$ and $\Gamma_{\epsilon}$. Consequently, these parameters were constrained to zero in our two-step estimation procedure.

\subsection{State variables}

Figure 2 displays the state variables for the three specifications estimated on USD data. The corresponding figure for the EUR market is similar and available in the online appendix. It is instructive to see the reaction of the state variables to the three most important shocks to the interbank money market during the sample period: the Bear Stearns near-bankruptcy on March 16, 2008, the Lehman Brothers bankruptcy on September 15, 2008, and the escalation of the European sovereign debt crisis often marked by the downgrade of Greece's debt to noninvestment grade status by Standard and Poor's on April 27, 2010. The figure shows that $\nu(t)$ increases leading up to the Bear Stearns near-default but quickly decreases after the take-over by J.P. Morgan. If anything, the opposite is true of $\xi(t)$. Immediately following the Lehman default, $\xi(t)$ spikes while $\nu(t)$ increases more gradually and does not reach its maximum until March 2009. Finally, with the escalation of the European sovereign debt crisis, $\nu(t)$ increases while $\xi(t)$ does not react. These dynamics hold true regardless of the model specification and suggest that an increase in the risk of credit quality deterioration was the main factor driving interbank risk around the first and third episode, while an increase in risk factors not directly related to default risk was the main driver in the aftermath of the Lehman default.

In order to better interpret the model's implications for current and future interbank default risk, we compute risk-neutral 3M and 6M expected default probabilities (EDPs) for an average bank within the current panel as well as for an average bank within the refreshed panel in five year's time. ${ }^{41}$ These are displayed in Figure 3 for the USD market, with the corresponding figure for the EUR market available in the online appendix. Taking the $\mathbb{A}(2,2,1)$ specification as an example, over our sample period, the spot 3M EDP averaged 0.09 percent but peaked at

\footnotetext{
${ }^{41}$ Specifically, we compute $E_{t}^{Q}\left[1_{t<\tau(t) \leq t+\Delta\}}\right]$ and $E_{t}^{Q}\left[1_{T<\tau(T) \leq T+\Delta\}}\right]$, where $\Delta$ equals $3 \mathrm{M}$ or $6 \mathrm{M}$ and $T$ equals $t$ plus 5 Y. Both expressions have analytical solutions in our affine framework.
} 
0.32 percent in March 2009 (Panel A2). Because of mean-reversion in the intensity of credit quality deterioration, the forward 3M EDP is much less volatile, averaging 0.07 percent and peaking at 0.11 percent. ${ }^{42}$ Until the Lehman Brothers default, the term structure of EDPs was mostly upward-sloping, implying that risk-neutral expectations were for interbank default risk to increase in the future. This contrasts with the downward-sloping spread term structure during this period (Figure 1), indicating an important role for non-default risk factors in determining short-term spreads. From the Lehman Brothers default until Fall 2009, the term structure of EDPs was downward-sloping, while it is again mostly upward-sloping during the last part of the sample period.

Similar dynamics are observed for the 6M EDPs (Panel B2). Note that the risk of credit quality deterioration combined with the potential for "refreshment" of the LIBOR panel implies that a strategy of lending for $6 \mathrm{M}$ to a LIBOR counterparty involves more default risk than lending for two consecutive $3 \mathrm{M}$ periods, as the latter strategy includes the option of switching to a more creditworthy counterparty after 3M. Indeed, the spot 6M EDP is consistently larger than the sum of the spot 3M EDP and the 3M forward 3M EDP for a refreshed panel. The sample mean of the former is 0.27 percent, while the sample mean of the latter sum is only 0.17 percent.

\subsection{Specification analysis}

For each of the model specifications, we compute the fitted OIS rates, interest rate spreads, and CDS spreads based on the filtered state variables. For each day in the sample and within each category - OIS, SPREAD $3 M, S P R E A D_{6 M}$, and $C D S$ - we then compute the root mean squared pricing errors (RMSEs) of the available rates or spreads, thereby constructing time series of RMSEs.

The first three rows of Panel A in Table 5 display the means of the RMSE time series in the USD market. The next two rows report the mean difference in RMSEs between two model specifications along with the associated $t$-statistics. Given that all specifications have two factors driving the OIS term structure, they obviously produce almost the same fit to OIS rates. However, they differ significantly in their fit to interest rate spreads and CDS spreads. $\mathbb{A}(2,2,1)$ has a significantly better fit than $\mathbb{A}(2,1,1)$ to the CDS term structure, with the mean

\footnotetext{
${ }^{42}$ Obviously, the expected default probabilities depend on our assumption of zero recovery (or $100 \%$ loss rate). As a rule of thumb, halving the loss rate is nearly the same as doubling the expected default probabilities.
} 
RMSE decreasing from 11.6 bp to $6.6 \mathrm{bp}$. It also appears to trade off a statistically significant

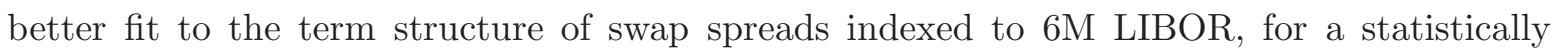
insignificant worse fit to the term structure of swap spreads indexed to 3M LIBOR. $\mathbb{A}(2,2,2)$ improves upon $\mathbb{A}(2,2,1)$ with a statistically significant better fit to the term structures of CDS spreads and swap spreads indexed to $6 \mathrm{M}$ LIBOR, and a marginally statistically significant better fit to the term structure of swap spreads indexed to 3M LIBOR. Economically, however, the improvement of $\mathbb{A}(2,2,2)$ over $\mathbb{A}(2,2,1)$ is modest (about 0.5 bp in terms of average RMSEs), and we do not expect more elaborate models to perform much better.

Panel B in Table 5 display the results for the EUR market, which are similar to those obtained for the USD market. ${ }^{43}$ In general, the model tends to have a slightly better fit to the EUR data than the USD data. This is also apparent from Table 4 where, for each specification, the estimated variance on the pricing errors is smaller for the EUR market.

More information about pricing errors are provided in the online appendix, where we display RMSEs for each point on the term structures of OIS rates, interest rate spreads, and CDS spreads. For the $\mathbb{A}(2,2,1)$ and $\mathbb{A}(2,2,2)$ specifications, the fit is generally rather uniform along the term structures, although in some cases we observe a deterioration in the fit at very short or very long maturities - an issue that is often encountered in term structure modeling. ${ }^{44}$

Since we value parsimony, in the following we will use the $\mathbb{A}(2,2,1)$ specification to analyze the term structure of interbank risk in more detail.

\subsection{Decomposing the term structure of interbank risk}

We measure the default component as the hypothetical swap spread that would materialize if default risk were the only risk factor in the interbank money market. This is computed by

\footnotetext{
${ }^{43}$ The main difference is that for the EUR market, the more elaborate specifications generate an improvement in the fit to the OIS term structure, which is statistically significant if still economically small.

${ }^{44}$ In our model, we assume that the OIS reference rate equals the average cost of unsecured overnight funding for LIBOR panel banks, which implies that the LIBOR-OIS spread goes to zero as maturity goes to zero. In principle, an interesting out-of-sample test of the model is the extent to which very short term LIBOR-OIS spreads implied by the model correspond to those observed in the data. In practice, however, very short-term LIBOR-OIS spreads are extremely noisy and display little correlation with longer term spreads. For instance, in the USD market, the shortest LIBOR maturity is overnight and the correlation between changes in the overnight LIBOR-FF spread and changes in the $3 \mathrm{M}$ and $6 \mathrm{M}$ LIBOR-OIS spreads are 0.08 and -0.01 , respectively. One would need to add additional factors to the model to capture the largely idiosyncratic behavior at the very short end of the spread curve.
} 
setting the residual term to one, $\Xi\left(t_{0}, T\right)=1$. The non-default component is then given by the difference between the fitted swap spread and the default-induced swap spread.

Table 6 displays, for each maturity, summary statistics of the two components. Focus first on the USD market. Panel A1 shows the decomposition of swap spreads indexed to 3M LIBOR. At the short end of the term structure, the default component is, on average, slightly smaller than the non-default component. As maturity increases, the default component, on average, first decreases and then increases for maturities beyond 4 years. On the other hand, the non-default component, on average, decreases rapidly with maturity. The upshot is that, as maturity increases, default increasingly becomes the dominant component. Panel A2 shows the decomposition of swap spreads indexed to 6M LIBOR. At the short end of the term structure, the default component is, on average, larger than the non-default component. Otherwise, the pattern is the same, with default increasingly becoming the dominant component as maturity increases.

Another observation from Table 6 is that both components are very volatile, particularly at the short end of the term structure. Figure 4 displays the time-series of the default and non-default components of the $3 \mathrm{M}$ and 6M LIBOR-OIS spreads (Panels A and B) and the $5 \mathrm{Y}$ swap spreads indexed to $3 \mathrm{M}$ or $6 \mathrm{M}$ LIBOR (Panels $\mathrm{C}$ and D). Consider first the money market spreads. Prior to the Lehman default, the default component constitutes a relatively small part of spreads, except for a brief period around the Bear Stearns near-default. In the aftermath of the Lehman default, the non-default component increases rapidly but then declines, while the default component increases gradually. The result is that by March 2009 and for the rest of the sample period, including the European sovereign debt crisis, spreads are almost exclusively driven by the default component. Consider next the 5Y swap spreads. Clearly, default is the overall more important component. Even prior to the Lehman default, the default component is the dominant driver of spreads. Immediately after the Lehman default both the default and non-default components increase after which the default component gradually becomes the exclusive driver of spreads.

Focus next on the EUR market. The summary statistics of the default and non-default components in Panels B1 and B2 in Table 6 are quite similar to those of the USD market. However, comparing Figure 5 with Figure 4 shows that EUR interbank risk is generally lower than USD interbank risk in the first half of the sample period, while the opposite is true in the second half. This is consistent with the observation that banks' exposures to US structured credit products was an important source of interbank risk in the first half of the sample period, 
while their exposures to European sovereign debt was a major source of interbank risk in the second half.

\subsection{Understanding the non-default component}

To understand the determinants of the non-default component, we investigate its relation with funding liquidity and market liquidity, which tend to be highly interconnected; see, e.g., Brunnermeier and Pedersen (2009).

Funding liquidity Given its over-the-counter structure, we do not have liquidity proxies that are specific to the market for unsecured interbank term funding. ${ }^{45}$ However, it is likely that liquidity in this market is correlated with liquidity in the market for secured term funding, which is another vital source of financing for banks. We consider two liquidity proxies for term repos. The first proxy is the spread between the 3M Agency MBS general collateral (GC) repo rate and the $3 \mathrm{M}$ Treasury GC repo rate. This measure reflects funding cost differentials between securities that differ in their market liquidity. ${ }^{46}$ The second proxy is the Fontaine and Garcia (2011) liquidity factor. This factor is estimated from the cross-section of on-the-run premia for Treasuries, which in turn depend on the funding advantage (or "specialness") of onthe-run Treasuries in the repo market; see, e.g., Duffie (1996) and Jordan and Jordan (1997). These liquidity proxies, denoted $\operatorname{Repospr}_{t}$ and $F G_{t}$, respectively, are displayed in Panels A and B in Figure 6.

Government bond market liquidity We consider two proxies for government bond market liquidity. The first proxy is the $\mathrm{Hu}$, Pan, and Wang (2010) liquidity factor, which is a daily aggregate of Treasury price deviations from "fair-value". Their argument is that lower liquidity allows more "noise" in the yield curve, as prices can deviate more from fundamental values before arbitrageurs step in to profit from mis-valuations. The second proxy is the spread between government bonds and government-sponsored agency bonds with lower liquidity but

\footnotetext{
${ }^{45}$ There does exist a trading platform for EUR interbank deposits, e-Mid. However, the maturities of the traded deposits are almost exclusively overnight (see Angelini, Nobili, and Picillo (2009)), while we are interested in liquidity measures for longer term deposits.

${ }^{46}$ In additional to wider spreads, larger initial margins (or "haircuts") would also indicate lower repo market liquidity. However, in contrasts to haircuts on structured product collateral, haircuts on Treasury and Agency MBS collateral were fairly stable throughout the crisis; see, e.g., Copeland, Martin, and Walker (2010) and Krishnamurthy, Nagel, and Orlov (2011).
} 
the same credit risk. Following Longstaff (2004), we use the spread between yields on Refcorp bonds and off-the-run Treasuries (specifically, we use the $10 \mathrm{Y}$ par yield spread). ${ }^{47}$ These liquidity proxies, denoted $H P W_{t}$ and $R C s p r_{t}$, respectively, are displayed in Panels $\mathrm{C}$ and $\mathrm{D}$ in Figure 6.

Corporate bond market liquidity As proxies for corporate bond market liquidity, we use the Dick-Nielsen, Feldhutter, and Lando (2012) liquidity factors. These factors are aggregates of several bond-specific liquidity and liquidity risk measures. We consider both their liquidity factor for the overall corporate bond market and their liquidity factor for bonds issued by financial institutions. The latter liquidity factor is particularly interesting since bond issuance (covered or uncovered) represents an important source of longer-term funding for banks. These liquidity proxies are displayed in Panels $\mathrm{E}$ and $\mathrm{F}$ in Figure 6 and are denote $D F L_{t}$ and DFLfin, respectively.

Approach We now relate the non-default component to these funding and market liquidity proxies. As in the previous sections, we focus on the $\mathbb{A}(2,2,1)$ specification, where the nondefault component is driven by $\xi_{t}$. Since $\xi_{t}$ captures the part of interbank risk that is unspanned by default risk, the relevant question is the extent to which $\xi_{t}$ is related to the parts of funding and market liquidity, which are unspanned by default risk. For this reason, we first regress the liquidity proxies on the first two principal components of the CDS term structure of the panel. $^{48}$ By construction, the regression residuals measure the variation in liquidity that is orthogonal to default risk. We then regress $\xi_{t}$ on these unspanned liquidity components.

Since $F G_{t}, H P W_{t}, D F L_{t}$, and DFLfin $n_{t}$ are only available until December 31, 2009, we use data up to this date in all regressions. Furthermore, since $F G_{t}, D F L_{t}$, and $D F L f i n_{t}$ are only available at a monthly frequency, we run all regressions on monthly data. ${ }^{49}$ Finally, to

\footnotetext{
${ }^{47}$ In her analysis of the 3M Euribor-OIS spread, Schwartz (2010) uses a similar spread between yields on KfW bonds (guaranteed by the German government) and German government bonds as a proxy for liquidity. We have experimented with this spread (again, the $10 \mathrm{Y}$ par yield spread) in our analysis of the EUR market, but found that the Refcorp-Treasury spread had better explanatory power.

${ }^{48}$ The first two principal components explain more than 99 percent of the variation in the CDS term structure of the LIBOR and EURIBOR panels. Regressing on a larger number of principal components does not change the results in any significant way.

${ }^{49}$ We convert daily time-series to monthly by averaging the daily observations over the month. This mirrors the construction of $D F L_{t}$, and DFLfint. Very similar results are obtained by using end-of-month observations.
} 
avoid spurious result due to the high persistence of the unspanned liquidity components, we run the second-step regressions in first differences. ${ }^{50}$

Results Table 7 displays pairwise correlations between monthly changes in the unspanned liquidity components and monthly changes in $\xi_{t}$. In general, the unspanned liquidity components are moderately correlated, except for $H P W_{t}$ and $R C s p r_{t}$ which are relatively highly correlated, and $D F L_{t}$ and $D F L f i n_{t}$ which are almost perfectly correlated.

Table 8 displays results from univariate and multivariate regressions of monthly changes in $\xi_{t}$ on monthly changes in the unspanned liquidity components. Consider first the USD market (Panel A of Table 8). In the univariate regressions, the coefficients on all the liquidity proxies are positive. $F G_{t}$ is marginally significant, $D F L_{t}$ and $D F L f i n_{t}$ are significant at conventional levels, and the rest are highly significant. Adjusted $R^{2} \mathrm{~s}$ lie between 0.10 and 0.49. In a multivariate regression with all liquidity proxies except $D F L f i n_{t},{ }^{51}$ the adjusted $R^{2}$ increases to 0.62 but, due to multicollinearity, several of the liquidity proxies become insignificant and have the wrong sign. Removing the least significant regressors results in a specification with only Repospr ${ }_{t}$ and $R C s p r_{t}$, both highly significant, and an adjusted $R^{2}$ of 0.64 .

Consider next the EUR market (Panel B of Table 8). The results are generally consistent with those of the USD market. In the univariate regressions the adjusted $R^{2} \mathrm{~S}$ vary between 0.03 and 0.44 , with all but the two corporate bond liquidity proxies being significant. In the regression specification with only Repospr $_{t}$ and $R C s p r_{t}$, both are highly significant and the adjusted $R^{2}$ reaches 0.70 .

Taken together, the results lend support to the conjecture that the non-default component of interbank risk largely captures liquidity effects not spanned by default risk.

\subsection{Pricing of interbank risk}

The model allows us to estimate the compensation that market participants require for bearing interbank risk. These results are necessarily tentative, since the relatively short sample period implies that the market price of risk parameters are estimated with some uncertainty.

\footnotetext{
${ }^{50}$ Unit root tests are available upon request.

${ }^{51} \mathrm{We}$ only include one of the corporate bond liquidity proxies in the multivariate regression, because of their near-perfect correlation.
} 
When discussing risk premia, it is important to distinguish between the swap market and the unsecured interbank money market. In both markets, there are premia associated with interest rate risk, variation in default risk, and unspanned liquidity risk. These premia are captured by the market prices of risk on the Wiener processes. However, in the unsecured interbank market, there is also a jump risk premium on the default event itself (to the extent that the mean loss rate differs under the objective and risk-neutral measures, see Yu (2002) and Jarrow, Lando, and Yu (2005)). Since our data set does not allow us to estimate the default event risk premium, we will focus on the risk premia that are available in the swap market.

As we are interested in the compensation for exposure to interbank risk, we consider a swap spread strategy consisting of receiving the fixed rate in an IRS indexed to 3M LIBOR and paying the fixed rate in an OIS of the same maturity. This strategy is expected to be approximately delta-neutral with respect to pure interest rate risk. The time- $t$ value of the strategy is $V_{t}^{S P R}=V_{t}^{I R S}-V_{t}^{O I S}$, where $V_{t}^{I R S}$ and $V_{t}^{O I S}$ denote the time- $t$ values of an IRS and OIS, respectively, from the perspective of the party who receives the fixed rate and pays the floating rate. The risk-neutral dynamics of the marked-to-market value of this strategy are given by ${ }^{52}$

$$
\begin{aligned}
d V_{t}^{S P R}= & r(t) V_{t}^{S P R} d t+\frac{\partial V_{t}^{S P R}}{\partial r} \sigma_{r} d W_{r}(t)+\frac{\partial V_{t}^{S P R}}{\partial \gamma} \sigma_{\gamma} d W_{\gamma}(t) \\
& +\frac{V_{t}^{I R S}}{\partial \nu} \sigma_{\nu} \sqrt{\nu(t)} d W_{\nu}(t)+\frac{V_{t}^{I R S}}{\partial \mu} \sigma_{\mu} \sqrt{\mu(t)} d W_{\mu}(t)+\frac{V_{t}^{I R S}}{\partial \xi} \sigma_{\xi} \sqrt{\xi(t)} d W_{\xi}(t) .(34)
\end{aligned}
$$

As expected, in our estimated model $\frac{\partial V_{t}^{S P R}}{\partial r}$ and $\frac{\partial V_{t}^{S P R}}{\partial \gamma}$ are negligible compared to $\frac{V_{t}^{I R S}}{\partial \nu}, \frac{V_{t}^{I R S}}{\partial \mu}$, and $\frac{V_{t}^{I R S}}{\partial \xi}$. Hence, the strategy has an almost pure exposure to interbank risk. With the market price of risk specification (30), the instantaneous Sharpe ratio is approximately given by

$$
S R_{t}^{S P R} \approx \frac{\frac{V_{t}^{I R S}}{\partial \nu} \sigma_{\nu} \Gamma_{\nu} \nu(t)+\frac{V_{t}^{I R S}}{\partial \mu} \sigma_{\mu} \Gamma_{\mu} \mu(t)+\frac{V_{t}^{I R S}}{\partial \xi} \sigma_{\xi} \Gamma_{\xi} \xi(t)}{\sqrt{\left(\frac{V_{t}^{I R S}}{\partial \nu}\right)^{2} \sigma_{\nu}^{2} \nu(t)+\left(\frac{V_{t}^{I R S}}{\partial \mu}\right)^{2} \sigma_{\mu}^{2} \mu(t)+\left(\frac{V_{t}^{I R S}}{\partial \xi}\right)^{2} \sigma_{\xi}^{2} \xi(t)}} .
$$

Figure 7 displays time-series of $S R_{t}^{S P R}$ at the $1 \mathrm{Y}, 5 \mathrm{Y}$, and $10 \mathrm{Y}$ swap maturities in the USD (results for the EUR market are similar). Due to their fast mean-reversion, $\nu(t)$ and $\xi(t)$ mainly affect near-term forward LIBOR rates. This implies that, for maturities beyond approximately one year, the spread strategy's loadings on $W_{\nu}(t)$ and $W_{\xi}(t)$ have very little dependence on maturity. In contrast, $\mu(t)$ also impacts long-term forward LIBOR rates, and

\footnotetext{
${ }^{52}$ It follows from (11) and (40) that $\frac{\partial V_{t}^{S P R}}{\partial \nu}=\frac{\partial V_{t}^{I R S}}{\partial \nu}, \frac{\partial V_{t}^{S P R}}{\partial \mu}=\frac{\partial V_{t}^{I R S}}{\partial \mu}$, and $\frac{\partial V_{t}^{S P R}}{\partial \xi}=\frac{\partial V_{t}^{I R S}}{\partial \xi}$.
} 
the spread strategy's loading on $W_{\mu}(t)$ increases with maturity over the entire maturity range. The sample averages of the market prices of risk on $W_{\nu}(t)$ and $W_{\mu}(t)$ are -0.28 and -0.20 , respectively, while we are not able to reliably estimate the market price of risk on $W_{\xi}(t)$, which is set to zero in our two-step estimation procedure, see end of Section 4.4. In the first half of the sample period, where unspanned liquidity risk is an important component of interbank risk, the strategy is primarily exposed to $W_{\xi}(t)$ for which there is no compensation. This is particularly the case for short swap maturities. Therefore, Sharpe ratios are low and increasing with swap maturity. The sole exceptions is the period around the Bear Stearns near-bankruptcy, where default risk briefly became the main driver of interbank risk. In the second half of the sample period, where default risk is the most important component of interbank risk, the strategy is mainly exposed to $W_{\nu}(t)$ and $W_{\mu}(t)$, and Sharpe ratios are, therefore, larger during this period. For instance, the instantaneous Sharpe ratio at the 5Y swap maturity is estimated to have averaged 0.35 from early 2009 to the end of the sample period. ${ }^{53}$

\section{$6 \quad$ Robustness checks}

Most of the results in Section 5 are based on the $\mathbb{A}(2,2,1)$ specification along with the $C D S_{\text {TrMean }}$ and $C D S_{\text {Median }}$ measures of interbank default risk in the USD and EUR markets, respectively. In this section, we investigate the robustness of our results to alternative model specifications and interbank default risk measures. Throughout, we focus on the USD market and the swap spread term structure indexed to 3M LIBOR. Decompositions are reported in Table 9, while plots of the default and non-default components are available in the online appendix. Conclusions for the spread term structure indexed to 6M LIBOR and for the EUR market are very similar. ${ }^{54}$

\footnotetext{
${ }^{53}$ For comparison, prior to the credit crisis, Duarte, Longstaff, and Yu (2007) report realized Sharpe ratios between 0.37 and 0.66 , depending on maturity, on spread arbitrage strategies between IRS and Treasuries.

${ }^{54}$ For the EUR market, only in the case where we use $C D S_{\text {iTrax }}$ to measure interbank default risk does the results differ noticeable from the baseline results. To some extent, this is due to the imperfect overlap between the set of underlying institutions in the iTraxx index and the EURIBOR panel. But primarily it is due to the fact that the index is only available for maturities of $5 \mathrm{Y}$ and $10 \mathrm{Y}$, leading to a less accurate identification of the process driving the risk of credit quality deterioration.
} 
$\mathbb{A}(\mathbf{2}, \mathbf{1 , 1})$ and $\mathbb{A}(\mathbf{2 , 2 , 2 )}$ We redo the decomposition of interbank risk using the $\mathbb{A}(2,1,1)$ and $\mathbb{A}(2,2,2)$ specifications (Panels $\mathrm{A}$ and $\mathrm{B}$, respectively, in Table 9). In both cases, the decomposition is very similar to the original one (which, for ease of comparison, is reproduced in the top panel in Table 9). One difference is that for the $\mathbb{A}(2,1,1)$ specification, the default component at the short end of the term structure is somewhat less volatile, which is not surprising given the one-factor nature of the risk of credit quality deterioration.

$\mathbb{A}(2,2,1)$ with $\kappa_{\boldsymbol{\lambda}}=\mathbf{0}$ We explore the effect of assuming that shocks to the credit quality of an average bank within the $t_{0}$-panel are permanent, which corresponds to setting $\kappa_{\lambda}=0$ in (24). We reestimate the $\mathbb{A}(2,2,1)$ specification subject to this constraint. The restricted specification has a significantly worse fit to the data with the average RMSE for $S P R E A D_{3 M}$, $S P R E A D_{6 M}$, and $C D S_{T r M e a n}$ increasing to $13.36 \mathrm{bp}, 11.72 \mathrm{bp}$, and $8.55 \mathrm{bp}$, respectively. Consequently, we do not consider that specification in greater detail.

$\mathbb{A}(2,2,1)$ with stochastic $\Lambda(t)$ Recall that the total default intensity of an average panel bank depends on $\Lambda(t)$ and the jump process representing credit quality deterioration, see (24). If $\Lambda(t)$ increases beyond the constant $5 \mathrm{bp}$ that we have assumed so far, the effect of the jump process has to decrease in order to still match a given level of CDS spreads. The model would then imply a smaller default component in the LIBOR-OIS and IRS-OIS spreads.

To investigate this possibility, while keeping the model tractable, we assume the existence of a latent default risk factor $\widetilde{\Lambda}(t)$, which evolves according to

$$
d \widetilde{\Lambda}(t)=\kappa_{\widetilde{\Lambda}}\left(\theta_{\widetilde{\Lambda}}-\widetilde{\Lambda}(t)\right) d t+\sigma_{\widetilde{\Lambda}} \sqrt{\widetilde{\Lambda}(t)} d W_{\widetilde{\Lambda}}(t),
$$

where $d W_{\widetilde{\Lambda}}(t)$ is another independent standard Brownian motion. $\widetilde{\Lambda}(t)$ generates variation in $\Lambda(t)$ and $\lambda\left(t_{0}, t\right)$ via

$$
\Lambda(t)=\Lambda\left(t_{0}\right)+\int_{t_{0}}^{t} \kappa_{\Lambda}(\widetilde{\Lambda}(s)-\Lambda(s)) d s
$$

and

$$
\lambda\left(t_{0}, t\right)=\Lambda\left(t_{0}\right)+\int_{t_{0}}^{t} \kappa_{\Lambda}\left(\widetilde{\Lambda}(s)-\lambda\left(t_{0}, s\right)\right) d s+\sum_{j=N\left(t_{0}\right)+1}^{N(t)} Z_{\lambda, j} .
$$

That is, $\Lambda(t)$ and $\lambda\left(t_{0}, t\right)$ fluctuate around the unobservable default risk factor. This specification preserves the feature that $\lambda\left(t_{0}, t\right)$ and $\Lambda(t)$ are identical until the first jump in $\lambda\left(t_{0}, t\right) .{ }^{55}$

\footnotetext{
${ }^{55}$ The resulting pricing formulas are available upon request. Note that with $\kappa_{\Lambda}=\kappa_{\lambda}$ and $\widetilde{\Lambda}(t)=\Lambda(t)=\Lambda$ this model reduces to our baseline model.
} 
To identify the additional dimensions of the model, we add the $1 \mathrm{~W}$ and 1M OIS-Treasury repo spreads to our original data set. These spreads directly depend on the $\Lambda(t)$-process. As discussed in Section 4.4, use of Treasury repo rates likely overstates $\Lambda(t)$ and this analysis, therefore, provides a lower bound on the effect of credit quality deterioration. ${ }^{56}$

The $\widetilde{\Lambda}(t)$-process is volatile $\left(\sigma_{\widetilde{\Lambda}}=0.46\right)$, displays fast mean-reversion $\left(\kappa_{\widetilde{\Lambda}}=7.65\right)$, and has a low long-run mean of $7 \mathrm{bp}$. The associated market price of risk parameter is imprecisely estimated and, therefore, is set to zero in the final estimation. With $\kappa_{\Lambda}$ estimated at 2.35, $\Lambda(t)$ tracks much of the variation in $\widetilde{\Lambda}(t)$. As expected, when $\Lambda(t)$ spikes, we observe a small decrease (increase) in the default (non-default) component of the LIBOR-OIS spread. However, because of the fast mean-reversion of $\Lambda(t)$ towards a low long-run mean, the impact on longer-term IRS-OIS spreads is negligible. The decomposition is reported in Panel $\mathrm{C}$ in Table 9. On average, the default (non-default) component is slightly smaller (larger) at the short end of the term structure, while indeed there is virtually no effect at longer maturities.

$\mathbb{A}(2,2,1)$ without LIBOR rates To address concerns about the integrity of LIBOR during parts of the sample period, we reestimate the $\mathbb{A}(2,2,1)$ specification without using LIBOR rates. In this case, interbank risk is identified purely from swap rates, which are determined in highly competitive markets. Panel D in Table 9 reports the decomposition, which is similar to the original one, except that the non-default component of the LIBOR-OIS spread is slightly smaller and less volatile.

$\mathbb{A}(2,2,1)$ with alternative default risk measures To investigate the sensitivity to possible liquidity effects in the CDS market, we reestimate the $\mathbb{A}(2,2,1)$ specification with the two liquidity-corrected default risk measures $C D S_{L I Q 1}$ and $C D S_{L I Q 2}$ described in Section 4.3. Panels $\mathrm{E}$ and $\mathrm{F}$ in Table 9 report the decomposition of the spread term structure in these two cases. At the short end of the term structure, the decomposition using $C D S_{L I Q 1}$ (Panel E) on average attributes a slightly smaller fraction of interbank risk to default risk compared with the original decomposition, while the decomposition using $C D S_{L I Q 2}$ (Panel F) on average attributes a slightly larger fraction of interbank risk to default risk. This is consistent with the fact that, on average, $C D S_{L I Q 1}<C D S_{T r M e a n}<C D S_{L I Q 2}$. At longer maturities, the differences are very small.

\footnotetext{
${ }^{56}$ Parameter estimates and state variables are available upon request. For the estimation, we assume a market price of risk process $\Gamma_{\widetilde{\Lambda}} \sqrt{\widetilde{\Lambda}(t)}$ such that $d W_{\widetilde{\Lambda}}(t)-\Gamma_{\widetilde{\Lambda}} \sqrt{\widetilde{\Lambda}(t)} d t$ becomes a standard Brownian motion under $P$.
} 


\section{Conclusion}

In this paper, we contribute to the rapidly growing literature on the interbank money market by studying the term structure of interbank risk. We follow most existing studies by measuring interbank risk by the spread between a LIBOR rate and the rate on an overnight indexed swap (OIS) of identical maturity. We show that the spread between the fixed rate on a long-term interest rate swap indexed to, say, 3M LIBOR, and a similar long-term OIS reflects risk-neutral expectations about future 3M LIBOR-OIS spreads. This allows us to infer a term structure of interbank risk from swap spreads of different maturities. We develop a dynamic term structure model with default risk in the interbank market that, in conjunction with information from the credit default swap market, allows us to decompose the term structure of interbank risk into default and non-default components. We apply the model to study interbank risk from the onset of the financial crisis in August 2007 until January 2011. We find that, on average, the fraction of total interbank risk due to default risk increases with maturity. At the short end of the term structure, the non-default component is important in the first half of the sample period and is correlated with various measures of funding liquidity and market liquidity. At longer maturities, the default component is the dominant driver of interbank risk throughout the sample period. We also provide tentative results indicating that swap market participants require compensation for exposure to variation in interbank default risk. Our results hold true in both the USD and EUR markets and are robust to different model parameterizations and measures of interbank default risk. Our analysis has implications for monetary and regulatory policy as well as for pricing, hedging, and risk-management in the interest rate swap market. 


\section{A Proof of (2)}

Discounting the integral equation (1) gives

$$
e^{-\int_{0}^{t} r(s) d s} V(t)=E_{t}^{Q}\left[e^{-\int_{0}^{T} r(s) d s} X+\int_{t}^{T} e^{-\int_{0}^{u} r(s) d s}\left(r(u)-r_{c}(u)\right) V(u) d u\right] .
$$

Hence

$$
M(t)=e^{-\int_{0}^{t} r(s) d s} V(t)+\int_{0}^{t} e^{-\int_{0}^{u} r(s) d s}\left(r(u)-r_{c}(u)\right) V(u) d u
$$

is a $Q$-martingale. We obtain

$$
d\left(e^{-\int_{0}^{t} r(s) d s} V(t)\right)=-\left(r(t)-r_{c}(t)\right)\left(e^{-\int_{0}^{t} r(s) d s} V(t)\right) d t+d M(t)
$$

Integration by parts then implies

$$
\begin{aligned}
& d\left(e^{-\int_{0}^{t} r_{c}(s) d s} V(t)\right)=d\left(e^{\int_{0}^{t}\left(r(s)-r_{c}(s)\right) d s} e^{-\int_{0}^{t} r(s) d s} V(t)\right) \\
& =e^{-\int_{0}^{t} r(s) d s} V(t) e^{\int_{0}^{t}\left(r(s)-r_{c}(s)\right) d s}\left(r(t)-r_{c}(t)\right) d t \\
& +e^{\int_{0}^{t}\left(r(s)-r_{c}(s)\right) d s}\left(-\left(r(t)-r_{c}(t)\right)\left(e^{-\int_{0}^{t} r(s) d s} V(t)\right) d t+d M(t)\right) \\
& =e^{\int_{0}^{t}\left(r(s)-r_{c}(s)\right) d s} d M(t) .
\end{aligned}
$$

Hence $e^{-\int_{0}^{t} r_{c}(s) d s} V(t)$ is a $Q$-martingale, and since $V(T)=X$ we conclude that

$$
e^{-\int_{0}^{t} r_{c}(s) d s} V(t)=E_{t}^{Q}\left[e^{-\int_{0}^{T} r_{c}(s) d s} X\right]
$$

which proves (2).

\section{B Extended doubly stochastic framework}

Here, we briefly recap and extend the standard doubly stochastic framework for modeling default times in our setting. ${ }^{57}$ The main aspect of our extension is that we can incorporate an arbitrary number of default times in one framework. We assume that the filtered probability space $\left(\Omega, \mathcal{F}, \mathcal{F}_{t}, Q\right)$ carries an i.i.d. sequence of standard exponential random variables $\varepsilon\left(t_{0}\right) \sim$ $\operatorname{Exp}(1)$, for $t_{0} \geq 0$, which are independent of $\mathcal{F}_{\infty}$. For every $t_{0} \geq 0$, we let $\lambda\left(t_{0}, t\right)$ be a nonnegative $\mathcal{F}_{t}$-adapted intensity process with the property

$$
\int_{t_{0}}^{t} \lambda\left(t_{0}, s\right) d s<\infty
$$

\footnotetext{
${ }^{57}$ Standard references are Duffie and Singleton (2003) and Lando (2004).
} 
for all finite $t \geq t_{0}$. We then define the random time

$$
\tau\left(t_{0}\right)=\inf \left\{t>t_{0} \mid \int_{t_{0}}^{t} \lambda\left(t_{0}, s\right) d s \geq \varepsilon\left(t_{0}\right)\right\}>t_{0} .
$$

Note that $\tau\left(t_{0}\right)$ is not an $\mathcal{F}_{t}$-stopping time but becomes a stopping time with respect to the enlarged filtration $\mathcal{G}_{t}=\mathcal{F}_{t} \vee \mathcal{H}_{t}$ where $\mathcal{H}_{t}=\vee_{t_{0} \geq 0} \sigma\left(H\left(t_{0}, s\right) \mid s \leq t\right)$ is the filtration generated by all $\tau\left(t_{0}\right)$-indicator processes $H\left(t_{0}, t\right)=1_{\left\{\tau\left(t_{0}\right) \leq t\right\}}$. The $\mathcal{G}_{t^{-}}$-stopping times $\tau\left(t_{0}\right)$ are then $\mathcal{F}_{t}$-doubly stochastic in the sense that

$$
E^{Q}\left[Y 1_{\left\{\tau\left(t_{0}\right)>T\right\}} \mid \mathcal{G}_{t_{0}}\right]=E_{t_{0}}^{Q}\left[Y e^{-\int_{t_{0}}^{T} \lambda\left(t_{0}, s\right) d s}\right]
$$

for all $\mathcal{F}_{T}$-measurable nonnegative random variables $Y$, see e.g. Filipović (2009, Lemma 12.2).

\section{Pricing formulas for the affine model}

In this section we derive the pricing formulas for the affine model used in this paper. It is evident from the system of stochastic differential equations composed of (23), (26), and (29) that the partial state vectors $(r(t), \gamma(t))^{\top},\left(\nu(t), \mu(t), \lambda\left(t_{0}, t\right)\right)^{\top}$, and $(\xi(t), \epsilon(t))^{\top}$ form independent autonomous affine jump-diffusion processes. Hence the subsequent exponentialaffine expressions (40), (42), (46) follow directly from the general affine transform formula in Duffie, Filipović, and Schachermayer (2003, Section 2), and the fact that $r_{c}(t)=r(t)+\Lambda$, see (18). The following formulas are for the full $\mathbb{A}(2,2,2)$ model. The nested versions, $\mathbb{A}(2,2,1)$ and $\mathbb{A}(2,1,1)$, are obtained by setting the respective model parameters, $\kappa_{\epsilon}, \theta_{\epsilon}, \sigma_{\epsilon}$ and $\kappa_{\mu}, \theta_{\mu}, \sigma_{\mu}$, equal to zero, and setting $\epsilon(t) \equiv \theta_{\xi}$ and $\mu(t) \equiv \theta_{\nu}$, respectively.

Lemma C.1. The time $t$ price of the collateralized zero-coupon bond maturing at $T$ equals

$$
\begin{aligned}
P_{c}(t, T) & =E_{t}^{Q}\left[e^{-\int_{t}^{T} r_{c}(s) d s}\right] \\
& =\exp \left[A(T-t)+B_{r}(T-t) r(t)+B_{\gamma}(T-t) \gamma(t)\right]
\end{aligned}
$$

where the functions $A$ and $B=\left(B_{r}, B_{\Lambda}\right)^{\top}$ solve the system of Riccati equations

$$
\begin{aligned}
\partial_{\tau} A(\tau) & =\frac{\sigma_{r}^{2}}{2} B_{r}(\tau)^{2}+\rho \sigma_{r} \sigma_{\gamma} B_{r}(\tau) B_{\gamma}(\tau)+\frac{\sigma_{\gamma}^{2}}{2} B_{\gamma}(\tau)^{2}+\kappa_{\gamma} \theta_{\gamma} B_{\gamma}(\tau)-\Lambda \\
\partial_{\tau} B_{r}(\tau) & =-\kappa_{r} B_{r}(\tau)-1 \\
\partial_{\tau} B_{\gamma}(\tau) & =-\kappa_{\gamma} B_{\gamma}(\tau)+\kappa_{r} B_{r}(\tau) \\
A(0) & =0, \quad B(0)=0 .
\end{aligned}
$$


Lemma C.2. The time $t_{0}$-value of an unsecured loan with notional 1 in (16) equals

$$
\begin{aligned}
B\left(t_{0}, T\right) & =E_{t_{0}}^{Q}\left[e^{-\int_{t_{0}}^{T}\left(r(s)+\lambda\left(t_{0}, s\right)\right) d s}\right] \\
& =P_{c}\left(t_{0}, T\right) \exp \left[C\left(T-t_{0}\right)+D_{\nu}\left(T-t_{0}\right) \nu\left(t_{0}\right)+D_{\mu}\left(T-t_{0}\right) \mu\left(t_{0}\right)+D_{\lambda}\left(T-t_{0}\right) \Lambda\right]
\end{aligned}
$$

where the functions $C$ and $D=\left(D_{\nu}, D_{\mu}, D_{\lambda}\right)^{\top}$ solve the system of Riccati equations

$$
\begin{aligned}
\partial_{\tau} C(\tau) & =\kappa_{\mu} \theta_{\mu} D_{\mu}(\tau)+\kappa_{\lambda} \Lambda D_{\lambda}(\tau)+\Lambda \\
\partial_{\tau} D_{\nu}(\tau) & =\frac{\sigma_{\nu}^{2}}{2} D_{\nu}(\tau)^{2}-\kappa_{\nu} D_{\nu}(\tau)+\frac{D_{\lambda}(\tau)}{\zeta_{\lambda}-D_{\lambda}(\tau)} \\
\partial_{\tau} D_{\mu}(\tau) & =\frac{\sigma_{\mu}^{2}}{2} D_{\mu}(\tau)^{2}-\kappa_{\mu} D_{\mu}(\tau)+\kappa_{\nu} D_{\nu}(\tau) \\
\partial_{\tau} D_{\lambda}(\tau) & =-\kappa_{\lambda} D_{\lambda}(\tau)-1 \\
C(0) & =0, \quad D(0)=0 .
\end{aligned}
$$

Proof. We write

$$
\begin{aligned}
B\left(t_{0}, T\right) & =E_{t_{0}}^{Q}\left[e^{-\int_{t_{0}}^{T}\left(r_{c}(s)-\Lambda+\lambda\left(t_{0}, s\right)\right) d s}\right] \\
& =E_{t_{0}}^{Q}\left[e^{-\int_{t_{0}}^{T} r_{c}(s) d s}\right] E_{t_{0}}^{Q}\left[e^{-\int_{t_{0}}^{T}\left(\lambda\left(t_{0}, s\right)-\Lambda\right) d s}\right] .
\end{aligned}
$$

Now the claim follows from the general affine transform formula in Duffie, Filipović, and Schachermayer (2003, Section 2). Note that $D_{\lambda}(\tau)<0$ for all $\tau>0$. Hence the rational function on the right hand side of the equation for $\partial_{\tau} D_{\nu}(\tau)$ is well defined and derived by

$$
\begin{aligned}
\int_{0}^{\infty}\left(e^{D_{\lambda}(\tau) \xi}-1\right) \zeta_{\lambda} e^{-\zeta_{\lambda} \xi} d \xi & =\zeta_{\lambda} \int_{0}^{\infty} e^{-\left(\zeta_{\lambda}-D_{\lambda}(\tau)\right) \xi} d \xi-1 \\
& =\frac{\zeta_{\lambda}}{\zeta_{\lambda}-D_{\lambda}(\tau)}-1 \\
& =\frac{D_{\lambda}(\tau)}{\zeta_{\lambda}-D_{\lambda}(\tau)}
\end{aligned}
$$

We obtain the following exponential affine expression for the $\left(T-t_{0}\right)$-maturity LIBOR rate $L\left(t_{0}, T\right)$.

Corollary C.3. The $\left(T-t_{0}\right)$-maturity LIBOR rate given in (17) equals

$$
\begin{aligned}
L\left(t_{0}, T\right)= & \frac{1}{T-t_{0}}\left(P_{c}\left(t_{0}, T\right)^{-1} \exp \left[-C\left(T-t_{0}\right)-D_{\nu}\left(T-t_{0}\right) \nu\left(t_{0}\right)-D_{\mu}\left(T-t_{0}\right) \mu\left(t_{0}\right)-D_{\lambda}\left(T-t_{0}\right) \Lambda\right]-1\right) \\
& \times \exp \left[-E\left(T-t_{0}\right)-F_{\xi}\left(T-t_{0}\right) \xi\left(t_{0}\right)-F_{\epsilon}\left(T-t_{0}\right) \epsilon\left(t_{0}\right)\right]
\end{aligned}
$$


with $C\left(T-t_{0}\right)$ and $D\left(T-t_{0}\right)$ given in Lemma $C .2$, and where the functions $E$ and $F=\left(F_{\xi}, F_{\epsilon}\right)^{\top}$ solve the Riccati equations

$$
\begin{aligned}
\partial_{\tau} E(\tau) & =\kappa_{\epsilon} \theta_{\epsilon} F_{\epsilon}(\tau) \\
\partial_{\tau} F_{\xi}(\tau) & =\frac{\sigma_{\xi}^{2}}{2} F_{\xi}(\tau)^{2}-\kappa_{\xi} F_{\xi}(\tau)-1 \\
\partial_{\tau} F_{\epsilon}(\tau) & =\frac{\sigma_{\epsilon}^{2}}{2} F_{\epsilon}(\tau)^{2}-\kappa_{\epsilon} F_{\epsilon}(\tau)+\kappa_{\xi} F_{\xi}(\tau) \\
E(0) & =0, \quad F(0)=0 .
\end{aligned}
$$

Proof. In view of (27) and the affine transform formula in Duffie, Filipović, and Schachermayer (2003, Section 2), the multiplicative residual term is given by

$$
\frac{1}{\Xi\left(t_{0}, T\right)}=\exp \left[E\left(T-t_{0}\right)+F_{\xi}\left(T-t_{0}\right) \xi\left(t_{0}\right)+F_{\epsilon}\left(T-t_{0}\right) \epsilon\left(t_{0}\right)\right]
$$

where the functions $E$ and $F=\left(F_{\xi}, F_{\epsilon}\right)^{\top}$ solve the Riccati equations (45). The corollary now follows from (17) and Lemma C.2.

In view of (7) we also need a closed form expression for

$$
I=E_{t}^{Q}\left[e^{-\int_{t}^{T} r_{c}(s) d s}\left(T-t_{0}\right) L\left(t_{0}, T\right)\right]
$$

for time points $t \leq t_{0}<T$. Using the tower property of conditional expectations we calculate

$$
\begin{aligned}
I= & E_{t}^{Q}\left[e^{-\int_{t}^{t_{0}} r_{c}(s) d s} E_{t_{0}}^{Q}\left[e^{-\int_{t_{0}}^{T} r_{c}(s) d s}\right]\left(T-t_{0}\right) L\left(t_{0}, T\right)\right] \\
= & E_{t}^{Q}\left[e^{-\int_{t}^{t_{0}} r_{c}(s) d s} P_{c}\left(t_{0}, T\right)\left(T-t_{0}\right) L\left(t_{0}, T\right)\right] \\
= & \left(E_{t}^{Q}\left[e^{-\int_{t}^{t_{0}} r_{c}(s) d s} \exp \left[-C\left(T-t_{0}\right)-D_{\nu}\left(T-t_{0}\right) \nu\left(t_{0}\right)-D_{\mu}\left(T-t_{0}\right) \mu\left(t_{0}\right)-D_{\lambda}\left(T-t_{0}\right) \Lambda\right]\right]\right. \\
& \left.\quad-E_{t}^{Q}\left[e^{-\int_{t}^{t_{0}} r_{c}(s) d s} P_{c}\left(t_{0}, T\right)\right]\right) \\
& \quad \times E_{t}^{Q}\left[\exp \left[-E\left(T-t_{0}\right)-F_{\xi}\left(T-t_{0}\right) \xi\left(t_{0}\right)-F_{\epsilon}\left(T-t_{0}\right) \epsilon\left(t_{0}\right)\right]\right] \\
= & \left(P_{c}\left(t, t_{0}\right) e^{-C\left(T-t_{0}\right)-D_{\lambda}\left(T-t_{0}\right) \Lambda} E_{t}^{Q}\left[\exp \left[-D_{\nu}\left(T-t_{0}\right) \nu\left(t_{0}\right)-D_{\mu}\left(T-t_{0}\right) \mu\left(t_{0}\right)\right]\right]-P_{c}(t, T)\right) \\
& \times E_{t}^{Q}\left[\exp \left[-E\left(T-t_{0}\right)-F_{\xi}\left(T-t_{0}\right) \xi\left(t_{0}\right)-F_{\epsilon}\left(T-t_{0}\right) \epsilon\left(t_{0}\right)\right]\right] .
\end{aligned}
$$

The conditional expectations on the right hand side of the last equality can easily be obtained in closed form using the affine transform formula in Duffie, Filipović, and Schachermayer (2003, Section 2).

It remains to be checked whether the above conditional expectations are well defined. Sufficient admissibility conditions on the model parameters are provided by the following lemma, the proof of which is in the online appendix. 
Lemma C.4. (i) Suppose $\kappa_{\lambda} \geq 0$, and define

$$
\begin{aligned}
\Theta_{\nu} & =\sqrt{\kappa_{\nu}^{2}+2 \frac{\sigma_{\nu}^{2}}{\zeta_{\lambda} \kappa_{\lambda}+1}}, \\
C_{\nu} & =\frac{\frac{2}{\zeta_{\lambda} \kappa_{\lambda}+1}\left(e^{\Theta_{\nu}\left(T-t_{0}\right)}-1\right)}{\Theta_{\nu}\left(e^{\Theta_{\nu}\left(T-t_{0}\right)}+1\right)+\kappa_{\nu}\left(e^{\Theta_{\nu}\left(T-t_{0}\right)}-1\right)}, \\
\Theta_{\mu} & =\sqrt{\kappa_{\mu}^{2}+2 \sigma_{\mu}^{2} \kappa_{\nu} C_{\nu}}, \\
C_{\mu} & =\frac{2 \kappa_{\nu} C_{\nu}\left(e^{\Theta_{\mu}\left(T-t_{0}\right)}-1\right)}{\Theta_{\mu}\left(e^{\Theta_{\mu}\left(T-t_{0}\right)}+1\right)+\kappa_{\mu}\left(e^{\Theta_{\mu}\left(T-t_{0}\right)}-1\right)} .
\end{aligned}
$$

If

$$
\kappa_{\nu}>\frac{1}{2} \sigma_{\nu}^{2} C_{\nu}
$$

and

$$
\begin{aligned}
& \kappa_{\mu} \geq \sigma_{\mu}^{2} C_{\mu} e^{-\frac{\kappa_{\mu}}{2} \tau^{*}} \\
&+\frac{4 \kappa_{\nu}^{2} \sigma_{\mu}^{2}}{\kappa_{\mu} \sigma_{\nu}^{2}}\left({ }_{2} F_{1}\left(1, \frac{\kappa_{\mu}}{2 \kappa_{\nu}} ; \frac{\kappa_{\mu}+2 \kappa_{\nu}}{2 \kappa_{\nu}} ; \frac{\left(\sigma_{\nu}^{2} C_{\nu}-2 \kappa_{\nu}\right) e^{\kappa_{\nu} \tau^{*}}}{\sigma_{\nu}^{2} C_{\nu}}\right)\right. \\
&\left.\quad-e^{-\frac{\kappa_{\mu}}{2} \tau^{*}}{ }_{2} F_{1}\left(1, \frac{\kappa_{\mu}}{2 \kappa_{\nu}} ; \frac{\kappa_{\mu}+2 \kappa_{\nu}}{2 \kappa_{\nu}} ; \frac{\sigma_{\nu}^{2} C_{\nu}-2 \kappa_{\nu}}{\sigma_{\nu}^{2} C_{\nu}}\right)\right)
\end{aligned}
$$

where ${ }_{2} F_{1}$ denotes the Gauss hypergeometric function and

$$
\tau^{*}=\frac{1}{\kappa_{\nu}} \log \max \left\{\frac{\left(2 \kappa_{\nu}-\sigma_{\nu}^{2} \frac{\kappa_{\mu}^{2}}{2 \kappa_{\nu} \sigma_{\mu}^{2}}\right) \frac{2 \kappa_{\nu} \sigma_{\mu}^{2}}{\kappa_{\mu}^{2}} C_{\nu}}{2 \kappa_{\nu}-\sigma_{\nu}^{2} C_{\nu}}, 1\right\},
$$

then

$$
E^{Q}\left[\exp \left[-D_{\nu}\left(T-t_{0}\right) \nu\left(t_{0}\right)-D_{\mu}\left(T-t_{0}\right) \mu\left(t_{0}\right)\right]\right]<\infty .
$$

(ii) Define

$$
\begin{aligned}
\Theta_{\xi} & =\sqrt{\kappa_{\xi}^{2}+2 \sigma_{\xi}^{2}}, \\
C_{\xi} & =\frac{2\left(e^{\Theta_{\xi}\left(T-t_{0}\right)}-1\right)}{\Theta_{\xi}\left(e^{\Theta_{\xi}\left(T-t_{0}\right)}+1\right)+\kappa_{\xi}\left(e^{\Theta_{\xi}\left(T-t_{0}\right)}-1\right)}, \\
\Theta_{\epsilon} & =\sqrt{\kappa_{\epsilon}^{2}+2 \sigma_{\epsilon}^{2} \kappa_{\xi} C_{\xi}}, \\
C_{\epsilon} & =\frac{2 \kappa_{\xi} C_{\xi}\left(e^{\Theta_{\epsilon}\left(T-t_{0}\right)}-1\right)}{\Theta_{\epsilon}\left(e^{\Theta_{\epsilon}\left(T-t_{0}\right)}+1\right)+\kappa_{\epsilon}\left(e^{\Theta_{\epsilon}\left(T-t_{0}\right)}-1\right)} .
\end{aligned}
$$

If conditions (49) and (50) hold for $C_{\nu}, \kappa_{\nu}, \sigma_{\nu}, C_{\mu}, \kappa_{\mu}, \sigma_{\mu}$ replaced by $C_{\xi}, \kappa_{\xi}, \sigma_{\xi}, C_{\epsilon}, \kappa_{\epsilon}, \sigma_{\epsilon}$, respectively, then

$$
E^{Q}\left[\exp \left[-F_{\xi}\left(T-t_{0}\right) \xi\left(t_{0}\right)-F_{\epsilon}\left(T-t_{0}\right) \epsilon\left(t_{0}\right)\right]\right]<\infty .
$$


Remark C.5. Note that $\tau^{*}=0$ if and only if $\frac{\kappa_{\mu}^{2}}{2 \kappa_{\nu} \sigma_{\mu}^{2}} \geq C_{\nu}$. In this case, (50) reads as $\kappa_{\mu} \geq \sigma_{\mu}^{2} C_{\mu}$, which is automatically satisfied as is shown at the end of the proof of Lemma C.4.

For the CDS coupon rate calculations, we need the respective exponential affine expressions for $(20),(21)$ and $(22)$. For $I_{1}\left(t_{0}, T\right)$ we obtain

$$
I_{1}\left(t_{0}, T\right)=\sum_{i=1}^{N}\left(t_{i}-t_{i-1}\right) e^{-\left(t_{i}-t_{0}\right) \Lambda} B\left(t_{0}, t_{i}\right) .
$$

In both formulas for $I_{2}\left(t_{0}, T\right)$ and $V_{\text {prot }}\left(t_{0}, T\right)$ the following expression shows up

$$
J\left(t_{0}, u\right)=E_{t_{0}}^{Q}\left[e^{-\int_{t_{0}}^{u}\left(r_{c}(s)+\lambda\left(t_{0}, s\right)\right) d s} \lambda\left(t_{0}, u\right)\right] .
$$

Lemma C.6. We have

$$
J\left(t_{0}, u\right)=\left(g\left(u-t_{0}\right)+h_{\nu}\left(u-t_{0}\right) \nu\left(t_{0}\right)+h_{\mu}\left(u-t_{0}\right) \mu\left(t_{0}\right)+h_{\lambda}\left(u-t_{0}\right) \Lambda\right) e^{-\left(u-t_{0}\right) \Lambda} B\left(t_{0}, u\right)
$$

where the functions $g$ and $h=\left(h_{\nu}, h_{\mu}, h_{\lambda}\right)^{\top}$ solve the linear inhomogeneous system of ordinary differential equations

$$
\begin{aligned}
\partial_{\tau} g(\tau) & =\kappa_{\mu} \theta_{\mu} h_{\mu}(\tau)+\kappa_{\lambda} \Lambda h_{\lambda}(\tau) \\
\partial_{\tau} h_{\nu}(\tau) & =\sigma_{\nu}^{2} D_{\nu}(\tau) h_{\nu}(\tau)-\kappa_{\nu} h_{\nu}(\tau)+\frac{\zeta_{\lambda} h_{\lambda}(\tau)}{\left(\zeta_{\lambda}-D_{\lambda}(\tau)\right)^{2}} \\
\partial_{\tau} h_{\mu}(\tau) & =\sigma_{\mu}^{2} D_{\mu}(\tau) h_{\mu}(\tau)-\kappa_{\mu} h_{\mu}(\tau)+\kappa_{\nu} h_{\nu}(\tau) \\
\partial_{\tau} h_{\lambda}(\tau) & =-\kappa_{\lambda} h_{\lambda}(\tau) \\
g(0) & =0, \quad h(0)=(0,0,1)^{\top} .
\end{aligned}
$$

and where the functions $D=\left(D_{\nu}, D_{\mu}, D_{\lambda}\right)^{\top}$ are given in Lemma C.2.

Proof. We first decompose $J\left(t_{0}, u\right)=P_{c}\left(t_{0}, u\right) I\left(t_{0}, u\right)$ with

$$
I\left(t_{0}, u\right)=E_{t_{0}}^{Q}\left[e^{-\int_{t_{0}}^{u} \lambda\left(t_{0}, s\right) d s} \lambda\left(t_{0}, u\right)\right]
$$

which we can compute by differentiating the respective moment generating function ${ }^{58}$ :

$$
I\left(t_{0}, u\right)=\left.\frac{d}{d v} E_{t_{0}}^{Q}\left[e^{-\int_{t_{0}}^{u} \lambda\left(t_{0}, s\right) d s} e^{v \lambda\left(t_{0}, u\right)}\right]\right|_{v=0} .
$$

\footnotetext{
${ }^{58}$ Note that the change of order of differentiation and expectation is justified by dominated convergence. Indeed, it follows from Duffie, Filipović, and Schachermayer (2003, Theorem 2.16) that $E_{t_{0}}^{Q}\left[e^{-\int_{t_{0}}^{u} \lambda\left(t_{0}, s\right) d s} e^{v \lambda\left(t_{0}, u\right)}\right]$ is finite for all $v$ in some neighborhood of zero.
} 
The affine transform formula in Duffie, Filipović, and Schachermayer (2003, Section 2), gives us

$$
\begin{aligned}
& E_{t_{0}}^{Q}\left[e^{-\int_{t_{0}}^{u} \lambda\left(t_{0}, s\right) d s} e^{v \lambda\left(t_{0}, u\right)}\right] \\
& \quad=\exp \left[G\left(u-t_{0}, v\right)+H_{\nu}\left(u-t_{0}, v\right) \nu\left(t_{0}\right)+H_{\mu}\left(u-t_{0}, v\right) \mu\left(t_{0}\right)+H_{\lambda}\left(u-t_{0}, v\right) \Lambda\right]
\end{aligned}
$$

where the functions $G$ and $H=\left(H_{\nu}, H_{\mu}, H_{\lambda}\right)^{\top}$ solve the system of Riccati equations

$$
\begin{aligned}
\partial_{\tau} G(\tau, v) & =\kappa_{\mu} \theta_{\mu} H_{\mu}(\tau, v)+\kappa_{\lambda} \Lambda H_{\lambda}(\tau, v) \\
\partial_{\tau} H_{\nu}(\tau, v) & =\frac{\sigma_{\nu}^{2}}{2} H_{\nu}(\tau, v)^{2}-\kappa_{\nu} H_{\nu}(\tau, v)+\frac{H_{\lambda}(\tau, v)}{\zeta_{\lambda}-H_{\lambda}(\tau, v)} \\
\partial_{\tau} H_{\mu}(\tau, v) & =\frac{\sigma_{\mu}^{2}}{2} H_{\mu}(\tau, v)^{2}-\kappa_{\mu} H_{\mu}(\tau, v)+\kappa_{\nu} H_{\nu}(\tau, v) \\
\partial_{\tau} H_{\lambda}(\tau, v) & =-\kappa_{\lambda} H_{\lambda}(\tau, v)-1 \\
G(0, v) & =0, \quad H(0, v)=(0,0, v)^{\top} .
\end{aligned}
$$

Hence from (54) we obtain

$$
\begin{aligned}
I\left(t_{0}, u\right)= & \left(g\left(u-t_{0}\right)+h_{\nu}\left(u-t_{0}\right) \nu\left(t_{0}\right)+h_{\mu}\left(u-t_{0}\right) \mu\left(t_{0}\right)+h_{\lambda}\left(u-t_{0}\right) \Lambda\right) \\
& \times \exp \left[G\left(u-t_{0}, 0\right)+H_{\nu}\left(u-t_{0}, 0\right) \nu\left(t_{0}\right)+H_{\mu}\left(u-t_{0}, 0\right) \mu\left(t_{0}\right)+H_{\lambda}\left(u-t_{0}, 0\right) \Lambda\right]
\end{aligned}
$$

where $g(\tau)=\left.\frac{d}{d v} G(\tau, v)\right|_{v=0}$, and $h=\left(h_{\nu}, h_{\mu}, h_{\lambda}\right)^{\top}$ is given by $h(\tau)=\left.\frac{d}{d v} H(\tau, v)\right|_{v=0}$. Note that $G(\tau, 0)=C(\tau)-\tau \Lambda$ and $H(\tau, 0)=D(\tau)$, see Lemma C.2. Differentiating both sides of the system (55) in $v$ at $v=0$ shows that the functions $g$ and $h$ solve the linear inhomogeneous system of ordinary differential equations (53). Thus the lemma is proved.

\section{Maximum likelihood estimation}

\section{D.1 The state space form}

We cast the model in state space form, which consists of a measurement equation and a transition equation. The measurement equation describes the relation between the state variables and the OIS rates, interest rate spreads, and CDS spreads, while the transition equation describes the discrete-time dynamics of the state variables.

Let $X_{t}$ denote the vector of state variables. While the transition density of $X_{t}$ is unknown, its conditional mean and variance is known in closed form, since $X_{t}$ follows an affine diffusion 
process. We approximate the transition density with a Gaussian density with identical first and second moments, in which case the transition equation becomes

$$
X_{t}=\Phi_{0}+\Phi_{X} X_{t-1}+w_{t}, \quad w_{t} \sim N\left(0, Q_{t}\right),
$$

with $\Phi_{0}, \Phi_{X}$, and $Q_{t}$ given in closed form. ${ }^{59}$

The measurement equation is given by

$$
Z_{t}=h\left(X_{t}\right)+u_{t}, \quad u_{t} \sim N(0, \Omega),
$$

where $Z_{t}$ is the vector of OIS rates, interest rate spreads, and CDS spreads observed at time $t, h$ is the pricing function, and $u_{t}$ is a vector of iid. Gaussian pricing errors with covariance matrix $\Omega$. To reduce the number of parameters in $\Omega$, we follow usual practice in the empirical term structure literature in assuming that the pricing errors are cross-sectionally uncorrelated (that is, $\Omega$ is diagonal), and that the same variance, $\sigma_{e r r}^{2}$, applies to all pricing errors.

\section{D.2 The unscented Kalman filter}

If the pricing function were linear $h\left(X_{t}\right)=h_{0}+H X_{t}$, the Kalman filter would provide efficient estimates of the conditional mean and variance of the state vector. Let $\hat{X}_{t \mid t-1}=E_{t-1}\left[X_{t}\right]$ and $\hat{Z}_{t \mid t-1}=E_{t-1}\left[Z_{t}\right]$ denote the expectation of $X_{t}$ and $Z_{t}$, respectively, using information up to and including time $t-1$, and let $P_{t \mid t-1}$ and $F_{t \mid t-1}$ denote the corresponding error covariance matrices. Furthermore, let $\hat{X}_{t}=E_{t}\left[X_{t}\right]$ denote the expectation of $X_{t}$ including information at time $t$, and let $P_{t}$ denote the corresponding error covariance matrix. The Kalman filter consists of two steps: prediction and update. In the prediction step, $\hat{X}_{t \mid t-1}$ and $P_{t \mid t-1}$ are given by

$$
\begin{aligned}
\hat{X}_{t \mid t-1} & =\Phi_{0}+\Phi_{X} \hat{X}_{t-1} \\
P_{t \mid t-1} & =\Phi_{X} P_{t-1} \Phi_{X}^{\prime}+Q_{t},
\end{aligned}
$$

and $\hat{Z}_{t \mid t-1}$ and $F_{t \mid t-1}$ are in turn given by

$$
\begin{aligned}
& \hat{Z}_{t \mid t-1}=h\left(\hat{X}_{t \mid t-1}\right) \\
& F_{t \mid t-1}=H P_{t \mid t-1} H^{\prime}+\Omega .
\end{aligned}
$$

\footnotetext{
${ }^{59}$ Approximating the true transition density with a Gaussian makes this a QML procedure. While QML estimation has been shown to be consistent in many settings, it is in fact not consistent in a Kalman filter setting, since the conditional covariance matrix $Q_{t}$ in the recursions depends on the Kalman filter estimates of the volatility state variables rather than the true, but unobservable, values; see, e.g., Duan and Simonato (1999). However, simulation results in several papers have shown this issue to be negligible in practice.
} 
In the update step, the estimate of the state vector is refined based on the difference between predicted and observed quantities, with $\hat{X}_{t}=E_{t}\left[X_{t}\right]$ and $P_{t}$ given by

$$
\begin{aligned}
\hat{X}_{t} & =\hat{X}_{t \mid t-1}+W_{t}\left(Z_{t}-\hat{Z}_{t \mid t-1}\right) \\
P_{t} & =P_{t \mid t-1}-W_{t} F_{t \mid t-1} W_{t}^{\prime}
\end{aligned}
$$

where

$$
W_{t}=P_{t \mid t-1} H^{\prime} F_{t \mid t-1}^{-1}
$$

is the covariance between pricing and filtering errors.

In our setting, the pricing function is non-linear for all the instruments included in the estimation, and the Kalman filter has to be modified. Non-linear state space systems have traditionally been handled with the extended Kalman filter, which effectively linearizes the measure equation around the predicted state. However, in recent years the unscented Kalman filter has emerged as a very attractive alternative. Rather than approximating the measurement equation, it uses the true non-linear measurement equation and instead approximates the distribution of the state vector with a deterministically chosen set of sample points, called "sigma points", that completely capture the true mean and covariance of the state vector. When propagated through the non-linear pricing function, the sigma points capture the mean and covariance of the data accurately to the 2 nd order (3rd order for true Gaussian states) for any nonlinearity. ${ }^{60}$

More specifically, a set of $2 L+1$ sigma points and associated weights are selected according to the following scheme

$$
\begin{array}{ll}
\hat{\mathcal{X}}_{t \mid t-1}^{0}=\hat{X}_{t \mid t-1} & w^{0}=\frac{\kappa}{L+\kappa} \\
\hat{\mathcal{X}}_{t \mid t-1}^{i}=\hat{X}_{t \mid t-1}+\left(\sqrt{(L+\kappa) P_{t \mid t-1}}\right)_{i} & w^{i}=\frac{1}{2(L+\kappa)} \quad i=1, \ldots, L \\
\hat{\mathcal{X}}_{t \mid t-1}^{i}=\hat{X}_{t \mid t-1}-\left(\sqrt{(L+\kappa) P_{t \mid t-1}}\right)_{i} & w^{i}=\frac{1}{2(L+\kappa)} \quad i=L+1, \ldots, 2 L,
\end{array}
$$

where $L$ is the dimension of $\hat{X}_{t \mid t-1}, \kappa$ is a scaling parameter, $w^{i}$ is the weight associated with the $i$ 'th sigma-point, and $\left(\sqrt{(L+\kappa) P_{t \mid t-1}}\right)_{i}$ is the $i$ 'th column of the matrix square root.

\footnotetext{
${ }^{60}$ For comparison, the extended Kalman filter estimates the mean and covariance accurately to the 1st order. Note that the computational costs of the extended Kalman filter and the unscented Kalman filter are of the same order of magnitude.
} 
Then, in the prediction step, (60) and (61) are replaced by

$$
\begin{aligned}
& \hat{Z}_{t \mid t-1}=\sum_{i=0}^{2 L} w^{i} h\left(\hat{\mathcal{X}}_{t \mid t-1}^{i}\right) \\
& F_{t \mid t-1}=\sum_{i=0}^{2 L} w^{i}\left(h\left(\hat{\mathcal{X}}_{t \mid t-1}^{i}\right)-\hat{Z}_{t \mid t-1}\right)\left(h\left(\hat{\mathcal{X}}_{t \mid t-1}^{i}\right)-\hat{Z}_{t \mid t-1}\right)^{\prime}+\Omega .
\end{aligned}
$$

The update step is still given by (62) and (63), but with $W_{t}$ computed as

$$
W_{t}=\sum_{i=0}^{2 L} w^{i}\left(\hat{\mathcal{X}}_{t \mid t-1}^{i}-\hat{X}_{t \mid t-1}\right)\left(h\left(\hat{\mathcal{X}}_{t \mid t-1}^{i}\right)-\hat{Z}_{t \mid t-1}\right)^{\prime} F_{t \mid t-1}^{-1} .
$$

Finally, the log-likelihood function is given by

$$
\log L=-\frac{1}{2} \log 2 \pi \sum_{t=1}^{T} N_{t}-\frac{1}{2} \sum_{t=1}^{T} \log \left|F_{t \mid t-1}\right|-\frac{1}{2} \sum_{t=1}^{T}\left(Z_{t}-\hat{Z}_{t \mid t-1}\right)^{\prime} F_{t \mid t-1}^{-1}\left(Z_{t}-\hat{Z}_{t \mid t-1}\right),
$$

where $T$ is the number of observation dates, and $N_{t}$ is the dimension of $Z_{t}$. 


\begin{tabular}{|c|c|c|c|c|c|c|c|c|c|}
\hline & \multicolumn{9}{|c|}{ Maturity } \\
\hline & $3 \mathrm{M}$ & $6 \mathrm{M}$ & $1 \mathrm{Y}$ & $2 \mathrm{Y}$ & $3 \mathrm{Y}$ & $4 \mathrm{Y}$ & $5 \mathrm{Y}$ & $7 \mathrm{Y}$ & $10 \mathrm{Y}$ \\
\hline & \multicolumn{9}{|c|}{ Panel A: USD market } \\
\hline OIS & $\begin{array}{l}1.17 \\
(1.48)\end{array}$ & $\begin{array}{l}1.17 \\
(1.43)\end{array}$ & $\begin{array}{l}1.26 \\
(1.35)\end{array}$ & $\begin{array}{l}1.63 \\
(1.21)\end{array}$ & $\begin{array}{l}2.06 \\
(1.12)\end{array}$ & $\begin{array}{l}2.42 \\
(1.03)\end{array}$ & $\begin{array}{l}2.72 \\
(0.96)\end{array}$ & & $\begin{array}{l}3.17^{\dagger} \\
(0.55)\end{array}$ \\
\hline$S P R E A D_{3 M}$ & $\begin{array}{l}58.7 \\
(57.5)\end{array}$ & & $\begin{array}{l}51.2 \\
(34.6)\end{array}$ & $\begin{array}{l}43.8 \\
(23.2)\end{array}$ & $\begin{array}{l}39.0 \\
(17.2)\end{array}$ & $\begin{array}{l}35.4 \\
(14.0)\end{array}$ & $\begin{array}{l}32.5 \\
(11.9)\end{array}$ & & $\underset{(8.2)}{28.7^{\dagger}}$ \\
\hline$S P R E A D_{6 M}$ & & $\begin{array}{l}79.1 \\
(57.4)\end{array}$ & $\begin{array}{l}70.0 \\
(42.7)\end{array}$ & $\begin{array}{l}58.0 \\
(28.2)\end{array}$ & $\begin{array}{l}50.8 \\
(20.8)\end{array}$ & $\begin{array}{l}45.8 \\
(16.9)\end{array}$ & $\begin{array}{l}41.9 \\
(14.2)\end{array}$ & & $\begin{array}{l}38.1^{\dagger} \\
(7.7)\end{array}$ \\
\hline$C D S_{T r M e a n}$ & & $\begin{array}{l}67.8 \\
(46.5)\end{array}$ & $\begin{array}{l}70.2 \\
(44.9)\end{array}$ & $\begin{array}{l}78.7 \\
(41.2)\end{array}$ & $\begin{array}{l}85.3 \\
(37.9)\end{array}$ & $\begin{array}{l}93.4 \\
(37.0)\end{array}$ & $\begin{array}{l}99.1 \\
(35.9)\end{array}$ & $\begin{array}{l}102.1 \\
(34.5)\end{array}$ & $\begin{array}{l}104.8 \\
(33.3)\end{array}$ \\
\hline$C D S_{L I Q 1}$ & & $\begin{array}{l}61.1 \\
(41.9)\end{array}$ & $\begin{array}{l}63.2 \\
(40.4)\end{array}$ & $\begin{array}{l}70.9 \\
(37.1)\end{array}$ & $\begin{array}{l}76.8 \\
(34.1)\end{array}$ & $\begin{array}{l}84.1 \\
(33.3)\end{array}$ & $\begin{array}{l}89.2 \\
(32.3)\end{array}$ & $\begin{array}{l}91.9 \\
(31.0)\end{array}$ & $\begin{array}{l}94.3 \\
(30.0)\end{array}$ \\
\hline \multirow[t]{2}{*}{$C D S_{L I Q 2}$} & & $\begin{array}{l}78.7 \\
(55.1)\end{array}$ & $\begin{array}{l}82.9 \\
(53.4)\end{array}$ & $\begin{array}{l}91.2 \\
(48.4)\end{array}$ & $\begin{array}{l}98.8 \\
(45.0)\end{array}$ & $\begin{array}{l}106.2 \\
(43.0)\end{array}$ & $\begin{array}{l}113.1 \\
(42.0)\end{array}$ & $\begin{array}{l}114.6 \\
(40.7)\end{array}$ & $\begin{array}{l}116.6 \\
(39.2)\end{array}$ \\
\hline & \multicolumn{9}{|c|}{ Panel B: EUR market } \\
\hline$O I S$ & $\begin{array}{l}1.91 \\
(1.67)\end{array}$ & $\begin{array}{l}1.93 \\
(1.65)\end{array}$ & $\begin{array}{l}2.00 \\
(1.58)\end{array}$ & $\begin{array}{l}2.21 \\
(1.38)\end{array}$ & $\begin{array}{l}2.45 \\
(1.23)\end{array}$ & $\begin{array}{l}2.67 \\
(1.13)\end{array}$ & $\begin{array}{l}2.85 \\
(1.02)\end{array}$ & $\begin{array}{l}3.14 \\
(0.87)\end{array}$ & $\begin{array}{l}3.44 \\
(0.74)\end{array}$ \\
\hline$S P R E A D_{3 M}$ & $\begin{array}{l}58.7 \\
(35.6)\end{array}$ & & $\begin{array}{l}49.6 \\
(21.7)\end{array}$ & $\begin{array}{l}43.0 \\
(15.2)\end{array}$ & $\begin{array}{l}39.6 \\
(12.2)\end{array}$ & $\begin{array}{l}36.0 \\
(11.3)\end{array}$ & $\begin{array}{l}34.3 \\
(10.0)\end{array}$ & $\begin{array}{l}32.0 \\
(8.6)\end{array}$ & $\begin{array}{l}29.9 \\
(7.4)\end{array}$ \\
\hline$S P R E A D_{6 M}$ & & $\begin{array}{l}73.5 \\
(36.2)\end{array}$ & $\begin{array}{l}66.3 \\
(24.4)\end{array}$ & $\begin{array}{l}55.9 \\
(16.1)\end{array}$ & $\begin{array}{l}50.6 \\
(13.0)\end{array}$ & $\begin{array}{l}45.7 \\
(12.9)\end{array}$ & $\begin{array}{l}43.1 \\
(11.8)\end{array}$ & $\begin{array}{l}39.6 \\
(10.5)\end{array}$ & $\begin{array}{l}36.2 \\
(9.2)\end{array}$ \\
\hline$C D S_{\text {Median }}$ & & $\begin{array}{l}70.5 \\
(43.0)\end{array}$ & $\begin{array}{l}72.9 \\
(40.5)\end{array}$ & $\begin{array}{l}81.3 \\
(37.7)\end{array}$ & $\begin{array}{l}88.6 \\
(35.9)\end{array}$ & $\begin{array}{l}95.8 \\
(35.2)\end{array}$ & $\begin{array}{l}102.3 \\
(34.8)\end{array}$ & $\begin{array}{l}104.8 \\
(34.4)\end{array}$ & $\begin{array}{l}107.3 \\
(33.9)\end{array}$ \\
\hline$C D S_{L I Q 1}$ & & $\begin{array}{l}63.4 \\
(38.7)\end{array}$ & $\begin{array}{l}65.6 \\
(36.4)\end{array}$ & $\begin{array}{l}73.2 \\
(33.9)\end{array}$ & $\begin{array}{l}79.7 \\
(32.3)\end{array}$ & $\begin{array}{l}86.2 \\
(31.7)\end{array}$ & $\begin{array}{l}92.1 \\
(31.3)\end{array}$ & $\begin{array}{l}94.3 \\
(31.0)\end{array}$ & $\begin{array}{l}96.6 \\
(30.5)\end{array}$ \\
\hline$C D S_{L I Q 2}$ & & $\begin{array}{l}64.9 \\
(39.3)\end{array}$ & $\begin{array}{l}67.8 \\
(38.6)\end{array}$ & $\begin{array}{l}76.1 \\
(36.2)\end{array}$ & $\begin{array}{l}83.8 \\
(35.2)\end{array}$ & $\begin{array}{l}90.9 \\
(35.0)\end{array}$ & $\begin{array}{l}97.4 \\
(35.3)\end{array}$ & $\begin{array}{l}99.6 \\
(34.9)\end{array}$ & $\begin{array}{l}102.1 \\
(34.5)\end{array}$ \\
\hline$C D S_{i \operatorname{Trax} x}$ & & & & & & & $\begin{array}{l}104.0 \\
(39.0)\end{array}$ & & $\begin{array}{l}109.0 \\
(37.2)\end{array}$ \\
\hline
\end{tabular}

Notes: The table shows means and, in parentheses, standard deviations. SPREAD $3 M$ denotes the difference between the fixed rates on an IRS indexed to 3M LIBOR/EURIBOR and an OIS with the same maturity. $S P R E A D_{6 M}$ denotes the difference between the fixed rates on an IRS indexed to $6 \mathrm{M} \mathrm{LIBOR/EURIBOR}$ and an OIS with the same maturity. $C D S_{T r M e a n}$ and $C D S_{\text {Median }}$ are the CDS spread term structures for an average bank within the LIBOR and EURIBOR panels, respectively. $C D S_{L I Q 1}$, and $C D S_{L I Q 2}$ are the CDS spread term structures corrected for possible liquidity effects as described in the main text. $C D S_{i \operatorname{Traxx}}$ is the iTraxx Senior Financials CDS index. OIS rates are measured in percentages, while interest rate spreads and CDS spreads are measured in basis points. Each time series consists of 895 daily observations from August 09, 2007 to January 12, 2011, except those marked with $\dagger$ which consist of 643 daily observations from July 28, 2008 to January 12, 2011.

Table 1: Summary statistics of data 


\begin{tabular}{|c|c|c|c|c|c|c|}
\hline Bank & Currency & Mean CDS & Std CDS & Balance & Liquidity & Start date \\
\hline Bank of America & USD & 136 & 62 & 2230 & 203 & 09-Aug-2007 \\
\hline Bank of Tokyo Mitsubishi & USD & 71 & 28 & 1619 & 17 & 09-Aug-2007 \\
\hline Barclays & EUR & 111 & 46 & 2227 & 117 & 09-Aug-2007 \\
\hline Citigroup & USD & 201 & 122 & 1857 & 155 & 09-Aug-2007 \\
\hline Credit Suisse & EUR & 108 & 40 & 997 & 71 & 06-May-2008 \\
\hline Deutsche Bank & EUR & 95 & 32 & 2151 & 159 & 09-Aug-2007 \\
\hline HSBC & EUR & 75 & 29 & 2364 & 38 & 09-Aug-2007 \\
\hline J. P. Morgan Chase & USD & 92 & 36 & 2032 & 174 & 09-Aug-2007 \\
\hline Lloyds TSB & EUR & 122 & 58 & 925 & 58 & 09-Aug-2007 \\
\hline Rabobank & EUR & 77 & 39 & 871 & - & 09-Aug-2007 \\
\hline Royal Bank of Canada & USD & 75 & 36 & 606 & - & 09-Aug-2007 \\
\hline Societe Generale & EUR & 93 & 34 & 1467 & 66 & 09-Aug-2007 \\
\hline Norinchukin Bank & USD & 85 & 41 & 630 & 2 & 09-Aug-2007 \\
\hline RBS & EUR & 134 & 53 & 2739 & 117 & 09-Aug-2007 \\
\hline UBS & EUR & 120 & 62 & 1296 & 81 & 09-Aug-2007 \\
\hline WestLB & EUR & 118 & 41 & 347 & 16 & 09-Aug-2007 \\
\hline
\end{tabular}

Notes: The table displays data on the banks that are members of the LIBOR panel. For each bank, it shows the currency of the CDS contracts, the mean and standard deviation of the 5Y CDS spread in basis points per annum, the size of the balance sheet in billion USD equivalent as reported in the 2009 annual report, the average daily notional of CDS transactions in million USD equivalent as reported by the Depository Trust and Clearing Corporation, and the date from which the 5Y CDS contract is available in the Markit database.

Table 2: LIBOR panel 


\begin{tabular}{|c|c|c|c|c|c|c|}
\hline Bank & Currency & Mean CDS & Std CDS & Balance & Liquidity & Start date \\
\hline Erste Bank & EUR & 186 & 70 & 202 & 8 & 11-Aug-2008 \\
\hline Raiffeisen Zentralbank & EUR & 174 & 93 & 148 & 8 & 09-Aug-2007 \\
\hline Dexia Bank & EUR & 216 & 103 & 578 & 14 & 09-Aug-2007 \\
\hline $\mathrm{KBC}$ & EUR & 143 & 76 & 324 & 3 & 09-Aug-2007 \\
\hline Nordea & EUR & 74 & 31 & 508 & - & 09-Aug-2007 \\
\hline BNP-Paribas & EUR & 71 & 27 & 2058 & 72 & 09-Aug-2007 \\
\hline Societe Generale & EUR & 93 & 34 & 1024 & 66 & 09-Aug-2007 \\
\hline Natixis & EUR & 161 & 76 & 449 & 5 & 09-Aug-2007 \\
\hline Credit Agricole & EUR & 95 & 37 & 1557 & 74 & 09-Aug-2007 \\
\hline CIC & EUR & 92 & 34 & 236 & - & 09-Aug-2007 \\
\hline Landesbank Berlin & EUR & 108 & 32 & 144 & - & 14-Aug-2007 \\
\hline Bayerische Landesbank & EUR & 101 & 30 & 339 & 6 & 09-Aug-2007 \\
\hline Deutsche Bank & EUR & 95 & 32 & 1501 & 159 & 09-Aug-2007 \\
\hline WestLB & EUR & 118 & 41 & 242 & 16 & 09-Aug-2007 \\
\hline Commerzbank & EUR & 88 & 29 & 844 & 91 & 09-Aug-2007 \\
\hline DZ Bank & EUR & 106 & 32 & 389 & - & 09-Aug-2007 \\
\hline Genossenschaftsbank & EUR & 134 & 19 & 68 & - & 31-Oct-2008 \\
\hline Norddeutsche Landesbank & EUR & 99 & 29 & 239 & - & 09-Aug-2007 \\
\hline Landesbank Baden-Wurttemberg & EUR & 107 & 33 & 412 & - & 09-Aug-2007 \\
\hline Landesbank Hessen-Thuringen & EUR & 111 & 31 & 170 & - & 09-Aug-2007 \\
\hline National Bank of Greece & EUR & 342 & 286 & 113 & 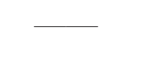 & 09-Aug-2007 \\
\hline Allied Irish Banks & EUR & 297 & 265 & 174 & 20 & 09-Aug-2007 \\
\hline Intesa Sanpaolo & EUR & 83 & 40 & 625 & 117 & 09-Aug-2007 \\
\hline Monte dei Paschi di Siena & EUR & 105 & 57 & 225 & 84 & 09-Aug-2007 \\
\hline Unicredit & EUR & 121 & 42 & 929 & 114 & 21-May-2008 \\
\hline ING Bank & EUR & 91 & 35 & 1164 & 35 & 09-Aug-2007 \\
\hline RBS & EUR & 145 & 20 & 1912 & 117 & 20-Aug-2010 \\
\hline Rabobank & EUR & 77 & 39 & 608 & - & 09-Aug-2007 \\
\hline Caixa Geral De Depositos & EUR & 164 & 132 & 121 & - & 09-Aug-2007 \\
\hline Banco Bilbao Vizcaya Argentaria & EUR & 114 & 62 & 535 & 133 & 09-Aug-2007 \\
\hline Banco Santander & EUR & 109 & 52 & 1111 & 156 & 24-Aug-2007 \\
\hline La Caixa & EUR & 178 & 87 & 272 & - & 09-Aug-2007 \\
\hline
\end{tabular}

Notes: Continued on next page.

Table 3: EURIBOR panel 


\begin{tabular}{lcccccc}
\hline Bank & Currency & Mean CDS & Std CDS & Balance & Liquidity & Start date \\
\hline Barclays & EUR & 111 & 46 & 1554 & 117 & 09-Aug-2007 \\
Danske Bank & EUR & 87 & 44 & 416 & 9 & 09 -Aug-2007 \\
Svenska Handelsbanken & EUR & 67 & 29 & 207 & 5 & $09-$ Aug-2007 \\
UBS & EUR & 120 & 62 & 904 & 81 & $09-A u g-2007$ \\
Citigroup & USD & 201 & 122 & 1296 & 155 & $09-A u g-2007$ \\
J.P. Morgan Chase & USD & 92 & 36 & 1418 & 174 & $09-A u g-2007$ \\
Bank of Tokyo Mitsubishi & USD & 71 & 28 & 1224 & 17 & $09-A u g-2007$ \\
\hline
\end{tabular}

Notes: The table displays data on the banks that are members of the EURIBOR panel. For each bank, it shows the currency of the CDS contracts, the mean and standard deviation of the 5Y CDS spread in basis points per annum, the size of the balance sheet in billion EUR equivalent as reported in the 2009 annual report, the average daily notional of CDS transactions in million USD equivalent as reported by the Depository Trust and Clearing Corporation, and the date from which the 5Y CDS contract is available in the Markit database.

Table 3: EURIBOR panel (cont.) 


\begin{tabular}{|c|c|c|c|c|c|c|}
\hline & \multicolumn{3}{|c|}{ USD market } & \multicolumn{3}{|c|}{ EUR market } \\
\hline & $\mathbb{A}(2,1,1)$ & $\mathbb{A}(2,2,1)$ & $\mathbb{A}(2,2,2)$ & $\mathbb{A}(2,1,1)$ & $\mathbb{A}(2,2,1)$ & $\mathbb{A}(2,2,2)$ \\
\hline$\kappa_{r}$ & $\begin{array}{l}0.1885 \\
(0.0143)\end{array}$ & $\begin{array}{l}0.2282 \\
(0.0205)\end{array}$ & $\begin{array}{l}0.3122 \\
(0.1322)\end{array}$ & $\begin{array}{l}0.2526 \\
(0.0068)\end{array}$ & $\begin{array}{l}0.2461 \\
(0.0042)\end{array}$ & $\begin{array}{l}0.2235 \\
(0.0031)\end{array}$ \\
\hline$\sigma_{r}$ & $\begin{array}{l}0.0055 \\
(0.0004)\end{array}$ & $\begin{array}{l}0.0054 \\
(0.0003)\end{array}$ & $\begin{array}{l}0.0054 \\
(0.0003)\end{array}$ & $\begin{array}{l}0.0053 \\
(0.0003)\end{array}$ & $\begin{array}{l}0.0053 \\
(0.0003)\end{array}$ & $\begin{array}{l}0.0051 \\
(0.0002)\end{array}$ \\
\hline$\kappa_{\gamma}$ & $\begin{array}{l}0.4667 \\
(0.0278)\end{array}$ & $\begin{array}{l}0.3864 \\
(0.0303)\end{array}$ & $\begin{array}{l}0.2836 \\
(0.1234)\end{array}$ & $\begin{array}{l}0.4703 \\
(0.0156)\end{array}$ & $\begin{array}{l}0.4663 \\
(0.0097)\end{array}$ & $\begin{array}{l}0.4566 \\
(0.0076)\end{array}$ \\
\hline$\theta_{\gamma}$ & $\begin{array}{l}0.1340 \\
(0.0031)\end{array}$ & $\begin{array}{l}0.1304 \\
(0.0022)\end{array}$ & $\begin{array}{l}0.1263 \\
(0.0020)\end{array}$ & $\begin{array}{l}0.0503 \\
(0.0003)\end{array}$ & $\begin{array}{l}0.0514 \\
(0.0003)\end{array}$ & $\begin{array}{l}0.0603 \\
(0.0009)\end{array}$ \\
\hline$\sigma_{\gamma}$ & $\begin{array}{l}0.2251 \\
(0.0180)\end{array}$ & $\begin{array}{l}0.1814 \\
(0.0166)\end{array}$ & $\begin{array}{l}0.1285 \\
(0.0547)\end{array}$ & $\begin{array}{l}0.0336 \\
(0.0026)\end{array}$ & $\begin{array}{l}0.0415 \\
(0.0029)\end{array}$ & $\begin{array}{l}0.0808 \\
(0.0044)\end{array}$ \\
\hline$\rho$ & $\begin{array}{c}-0.2115 \\
(0.2365)\end{array}$ & $\begin{array}{c}-0.2286 \\
(0.1771)\end{array}$ & $\begin{array}{c}-0.1694 \\
(0.1965)\end{array}$ & $\begin{array}{c}-0.2564 \\
(0.0831)\end{array}$ & $\begin{array}{c}-0.3198 \\
(0.0813)\end{array}$ & $\begin{array}{c}-0.2712 \\
(0.1319)\end{array}$ \\
\hline$\kappa_{\nu}$ & $\begin{array}{l}0.3268 \\
(0.0020)\end{array}$ & $\begin{array}{l}2.0977 \\
(0.0493)\end{array}$ & $\begin{array}{l}2.1843 \\
(0.0697)\end{array}$ & $\begin{array}{l}0.2603 \\
(0.0019)\end{array}$ & $\begin{array}{l}2.6835 \\
(0.0528)\end{array}$ & $\begin{array}{l}2.8773 \\
(0.0627)\end{array}$ \\
\hline$\sigma_{\nu}$ & $\begin{array}{l}0.3925 \\
(0.0085)\end{array}$ & $\begin{array}{l}0.6418 \\
(0.0577)\end{array}$ & $\begin{array}{l}0.5602 \\
(0.0537)\end{array}$ & $\begin{array}{l}0.3082 \\
(0.0069)\end{array}$ & $\begin{array}{l}0.6845 \\
(0.0488)\end{array}$ & $\begin{array}{l}0.5489 \\
(0.0396)\end{array}$ \\
\hline$\kappa_{\mu}$ & & $\begin{array}{l}0.0499 \\
(0.0067)\end{array}$ & $\begin{array}{l}0.0340 \\
(0.0072)\end{array}$ & & $\begin{array}{l}0.0156 \\
(0.0046)\end{array}$ & $\begin{array}{l}0.0152 \\
(0.0079)\end{array}$ \\
\hline$\theta_{\nu}$ or $\theta_{\mu}$ & $\begin{array}{l}0.2326 \\
(0.0019)\end{array}$ & $\begin{array}{l}0.3844 \\
(0.0258)\end{array}$ & $\begin{array}{l}0.4634 \\
(0.0635)\end{array}$ & $\begin{array}{l}0.2162 \\
(0.0012)\end{array}$ & $\begin{array}{l}0.6196 \\
(0.1268)\end{array}$ & $\begin{array}{l}0.6285 \\
(0.2555)\end{array}$ \\
\hline$\sigma_{\mu}$ & & $\begin{array}{l}0.2549 \\
(0.0074)\end{array}$ & $\begin{array}{l}0.2643 \\
(0.0074)\end{array}$ & & $\begin{array}{l}0.2049 \\
(0.0047)\end{array}$ & $\begin{array}{l}0.2144 \\
(0.0056)\end{array}$ \\
\hline$\kappa_{\lambda}$ & $\begin{array}{l}2.2595 \\
(0.0149)\end{array}$ & $\begin{array}{l}2.1878 \\
(0.0113)\end{array}$ & $\begin{array}{l}1.8242 \\
(0.0159)\end{array}$ & $\begin{array}{l}2.0701 \\
(0.0086)\end{array}$ & $\begin{array}{l}1.8452 \\
(0.0083)\end{array}$ & $\begin{array}{l}1.4773 \\
(0.0083)\end{array}$ \\
\hline$\kappa_{\xi}$ & $\begin{array}{l}7.1547 \\
(0.2180)\end{array}$ & $\begin{array}{l}6.2883 \\
(0.1114)\end{array}$ & $\begin{array}{l}7.2128 \\
(0.1187)\end{array}$ & $\begin{array}{l}6.0240 \\
(0.2739)\end{array}$ & $\begin{array}{l}5.7965 \\
(0.1982)\end{array}$ & $\begin{array}{l}6.7061 \\
(0.1504)\end{array}$ \\
\hline$\sigma_{\xi}$ & $\begin{array}{c}13.9675 \\
(0.4648)\end{array}$ & $\begin{array}{c}12.2294 \\
(0.2364)\end{array}$ & $\begin{array}{c}14.0501 \\
(0.2538)\end{array}$ & $\begin{array}{c}11.7578 \\
(0.5793)\end{array}$ & $\begin{array}{c}11.4319 \\
(0.4121)\end{array}$ & $\begin{array}{c}13.1116 \\
(0.3151)\end{array}$ \\
\hline$\kappa_{\epsilon}$ & & & $\begin{array}{l}1.3112 \\
(0.0594)\end{array}$ & & & $\begin{array}{l}0.4801 \\
(0.0252)\end{array}$ \\
\hline$\theta_{\xi}$ or $\theta_{\epsilon}$ & $\begin{array}{l}0.0001 \\
(0.0002)\end{array}$ & $\begin{array}{l}0.0010 \\
(0.0005)\end{array}$ & $\begin{array}{l}0.0002 \\
(0.0001)\end{array}$ & $\begin{array}{l}0.0001 \\
(0.0001)\end{array}$ & $\begin{array}{l}0.0027 \\
(0.0008)\end{array}$ & $\begin{array}{l}0.0001 \\
(0.0002)\end{array}$ \\
\hline$\sigma_{\epsilon}$ & & & $\begin{array}{l}1.9325 \\
(0.1089)\end{array}$ & & & $\begin{array}{l}0.6564 \\
(0.0415)\end{array}$ \\
\hline$\Gamma_{r}$ & $\begin{array}{c}-0.1499 \\
(0.1324)\end{array}$ & $\begin{array}{c}-0.1843 \\
(0.1562)\end{array}$ & 0 & 0 & 0 & 0 \\
\hline$\Gamma_{\gamma}$ & $\begin{array}{c}-0.2332 \\
(0.1154)\end{array}$ & $\begin{array}{c}-0.2123 \\
(0.1298)\end{array}$ & $\begin{array}{c}-0.1756 \\
(0.1256)\end{array}$ & $\begin{array}{c}-0.1348 \\
(0.0781)\end{array}$ & $\begin{array}{c}-0.1802 \\
(0.1187)\end{array}$ & $\begin{array}{c}-0.1599 \\
(0.0985)\end{array}$ \\
\hline$\Gamma_{\nu}$ & $\begin{array}{c}-0.3734 \\
(0.3354)\end{array}$ & $\begin{array}{c}-0.5687 \\
(0.4377)\end{array}$ & $\begin{array}{c}-0.6559 \\
(0.4177)\end{array}$ & $\begin{array}{c}-0.4675 \\
(0.4243)\end{array}$ & $\begin{array}{c}-0.5476 \\
(0.3911)\end{array}$ & $\begin{array}{c}-0.5210 \\
(0.3576)\end{array}$ \\
\hline$\Gamma_{\mu}$ & & $\begin{array}{c}-0.4467 \\
(0.1765)\end{array}$ & $\begin{array}{c}-0.5397 \\
(0.2332)\end{array}$ & & $\begin{array}{c}-0.3567 \\
(0.3124)\end{array}$ & 0 \\
\hline$\sigma_{e r r}(\mathrm{bp})$ & $\underset{(0.0235)}{10.4255}$ & $\begin{array}{l}8.6173 \\
(0.0223)\end{array}$ & $\begin{array}{l}8.1734 \\
(0.0216)\end{array}$ & $\underset{(0.0207)}{10.0982}$ & $\begin{array}{l}8.1173 \\
(0.0166)\end{array}$ & $\begin{array}{l}7.3956 \\
(0.0154)\end{array}$ \\
\hline $\log L \times 10^{-4}$ & -10.0436 & -9.5909 & -9.4830 & -11.1765 & -10.5827 & -10.3603 \\
\hline
\end{tabular}

Notes: The sample period is August 09, 2007 to January 12, 2011. Asymptotic standard errors are in parentheses. For identification purposes, we fix $\zeta_{\lambda}$ at 10 and $\Lambda$ at $5 \mathrm{bp}$. We constrain to zero those market price of risk parameters for which the absolute $t$-statistic does not exceed one. $\sigma_{\text {err }}$ denotes the standard deviation of pricing errors.

Table 4: Maximum-likelihood estimates 


\begin{tabular}{lcccc}
\hline & OIS & SPREAD $3 M$ & SPREAD & C \\
& & \multicolumn{2}{c}{ Panel A: USD } & market \\
\hline & 7.14 & 7.65 & 7.63 & \\
$\mathbb{A}(2,1,1)$ & 7.06 & 8.12 & 6.99 & 11.55 \\
$\mathbb{A}(2,2,1)$ & 7.02 & 7.65 & 6.37 & 6.62 \\
$\mathbb{A}(2,2,2)$ & $-0.09^{*}$ & 0.47 & $-0.63^{* *}$ & $-4.93^{* * *}$ \\
$\mathbb{A}(2,2,1)-\mathbb{A}(2,1,1)$ & $(-1.71)$ & $(0.99)$ & $(-2.51)$ & $(-4.95)$ \\
& -0.04 & $-0.47^{*}$ & $-0.62^{* * *}$ & $-0.43^{* * *}$ \\
$\mathbb{A}(2,2,2)-\mathbb{A}(2,2,1)$ & $(-0.79)$ & $(-1.78)$ & $(-5.72)$ & $(-3.26)$ \\
& & Panel B: EUR & market & \\
& 6.16 & 7.66 & 8.23 & 11.77 \\
$\mathbb{A}(2,1,1)$ & 5.93 & 7.83 & 7.16 & 7.07 \\
$\mathbb{A}(2,2,1)$ & 5.59 & 7.13 & 6.22 & 6.34 \\
$\mathbb{A}(2,2,2)$ & $-0.23^{* * *}$ & 0.17 & $-1.06^{* * *}$ & $-4.70^{* * *}$ \\
$\mathbb{A}(2,2,1)-\mathbb{A}(2,1,1)$ & $(-8.12)$ & $(0.63)$ & $(-4.04)$ & $(-4.62)$ \\
$\mathbb{A}(2,2,2)-\mathbb{A}(2,2,1)$ & $-0.34^{* * *}$ & -0.70 & $-0.95^{* * *}$ & $-0.73^{* * *}$ \\
\hline
\end{tabular}

Notes: The table reports means of the root mean squared pricing error (RMSE) time-series of OIS rates, interest rate spreads, and CDS spreads. SPREAD $3 M$ denotes the difference between the fixed rates on an IRS indexed to $3 \mathrm{M}$ LIBOR/EURIBOR and an OIS with the same maturity. SPREAD $6 M$ denotes the difference between the fixed rates on an IRS indexed to $6 \mathrm{M}$ LIBOR/EURIBOR and an OIS with the same maturity. Units are basis points. T-statistics, corrected for heteroscedasticity and serial correlation up to 50 lags using the method of Newey and West (1987), are in parentheses. *, **, and $* * *$ denote significance at the $10 \%, 5 \%$, and $1 \%$ levels, respectively. Each time series consists of 895 daily observations from August 09, 2007 to January 12, 2011 ,

Table 5: Comparing model specifications 


\begin{tabular}{|c|c|c|c|c|c|c|c|c|c|}
\hline & \multicolumn{9}{|c|}{ Maturity } \\
\hline & $3 \mathrm{M}$ & $6 \mathrm{M}$ & $1 Y$ & $2 \mathrm{Y}$ & $3 \mathrm{Y}$ & $4 \mathrm{Y}$ & $5 \mathrm{Y}$ & $7 \mathrm{Y}$ & $10 \mathrm{Y}$ \\
\hline & \multicolumn{9}{|c|}{ Panel A1: SPREAD 3 , USD market } \\
\hline Default & $\begin{array}{l}28.1 \\
(26.8)\end{array}$ & & $\begin{array}{l}25.2 \\
(17.2)\end{array}$ & $\begin{array}{l}24.0 \\
(12.5)\end{array}$ & $\begin{array}{l}23.8 \\
(10.7)\end{array}$ & $\begin{array}{l}23.9 \\
(9.8)\end{array}$ & $\begin{array}{l}24.1 \\
(9.2)\end{array}$ & & $\underset{(5.8)}{28.6^{\dagger}}$ \\
\hline \multirow[t]{2}{*}{ Non-default } & $\begin{array}{l}33.4 \\
(45.2)\end{array}$ & & $\begin{array}{l}20.4 \\
(27.3)\end{array}$ & $\begin{array}{l}10.6 \\
(14.1)\end{array}$ & $\begin{array}{l}7.2 \\
(9.5)\end{array}$ & $\begin{array}{l}5.5 \\
(7.3)\end{array}$ & $\begin{array}{l}4.5 \\
(6.0)\end{array}$ & & ${ }_{(3.5)}{ }^{\dagger}$ \\
\hline & \multicolumn{9}{|c|}{ Panel A2: SPREAD $6 M$, USD market } \\
\hline Default & & $\begin{array}{l}45.9 \\
(39.7)\end{array}$ & $\begin{array}{l}43.1 \\
(30.1)\end{array}$ & $\begin{array}{c}40.9 \\
(21.8)\end{array}$ & $\begin{array}{l}40.5 \\
(18.5)\end{array}$ & $\begin{array}{l}40.7 \\
(16.8)\end{array}$ & $\begin{array}{l}41.0 \\
(15.7)\end{array}$ & & ${ }_{(10.0)}^{48.6^{\dagger}}$ \\
\hline \multirow[t]{2}{*}{ Non-default } & & $\begin{array}{l}38.3 \\
(53.2)\end{array}$ & $\begin{array}{l}29.6 \\
(40.5)\end{array}$ & $\begin{array}{l}15.6 \\
(21.2)\end{array}$ & $\begin{array}{l}10.6 \\
(14.3)\end{array}$ & $\begin{array}{c}8.1 \\
(10.9)\end{array}$ & $\begin{array}{c}6.7 \\
(8.9)\end{array}$ & & ${ }_{(5.4)}^{2.9^{\dagger}}$ \\
\hline & \multicolumn{9}{|c|}{ Panel B1: SPREAD $D_{3 M}$, EUR market } \\
\hline Default & $\begin{array}{l}28.6 \\
(23.1)\end{array}$ & & $\begin{array}{l}24.2 \\
(13.7)\end{array}$ & $\begin{array}{l}22.5 \\
(10.0)\end{array}$ & $\begin{array}{l}22.1 \\
(8.8)\end{array}$ & $\begin{array}{l}22.1 \\
(8.2)\end{array}$ & $\begin{array}{l}22.2 \\
(7.9)\end{array}$ & $\begin{array}{l}22.7 \\
(7.5)\end{array}$ & $\begin{array}{l}23.4 \\
(7.2)\end{array}$ \\
\hline \multirow[t]{2}{*}{ Non-default } & $\begin{array}{l}30.5 \\
(34.1)\end{array}$ & & $\begin{array}{l}21.9 \\
(22.8)\end{array}$ & $\begin{array}{l}11.7 \\
(12.0)\end{array}$ & $\begin{array}{l}8.0 \\
(8.2)\end{array}$ & $\begin{array}{l}6.2 \\
(6.3)\end{array}$ & $\begin{array}{l}5.1 \\
(5.1)\end{array}$ & $\begin{array}{c}3.9 \\
(3.8)\end{array}$ & $\begin{array}{l}3.0 \\
(2.8)\end{array}$ \\
\hline & \multicolumn{9}{|c|}{ Panel B2: SPREAD 6 , EUR market } \\
\hline Default & & $\begin{array}{l}46.7 \\
(34.0)\end{array}$ & $\begin{array}{l}42.4 \\
(24.7)\end{array}$ & $\begin{array}{l}39.3 \\
(17.9)\end{array}$ & $\begin{array}{l}38.5 \\
(15.6)\end{array}$ & $\begin{array}{c}38.5 \\
(14.5)\end{array}$ & $\begin{array}{l}38.7 \\
(13.8)\end{array}$ & $\begin{array}{l}39.4 \\
(13.1)\end{array}$ & $\begin{array}{l}40.8 \\
(12.5)\end{array}$ \\
\hline Non-default & & $\begin{array}{l}34.1 \\
(36.1)\end{array}$ & $\begin{array}{l}31.0 \\
(31.2)\end{array}$ & $\begin{array}{c}17.3 \\
(17.1)\end{array}$ & $\begin{array}{c}11.9 \\
(11.7)\end{array}$ & $\begin{array}{l}9.2 \\
(8.9)\end{array}$ & $\begin{array}{c}7.6 \\
(7.3)\end{array}$ & $\begin{array}{c}5.8 \\
(5.4)\end{array}$ & $\begin{array}{l}4.5 \\
(4.0)\end{array}$ \\
\hline
\end{tabular}

Notes: The table shows the decomposition of the spread term structures using the $\mathbb{A}(2,2,1)$ specification and the $C D S_{T r M e a n}$ and $C D S_{M e d i a n}$ measures of interbank default risk in the USD and EUR markets, respectively. Each spread is decomposed into a default and a non-default component and the table displays means and, in parentheses, standard deviations of the two components. SPREAD $3 M$ and $S P R E A D_{6 M}$ denote the spread term structures indexed to $3 \mathrm{M}$ and $6 \mathrm{M}$ LIBOR/EURIBOR, respectively. Units are basis points. Each time series consists of 895 daily observations from August 09, 2007 to January 12, 2011, except those marked with $\dagger$ which consist of 643 daily observations from July 28, 2008 to January 12, 2011.

Table 6: Decomposition of the term structure of interbank risk 


\begin{tabular}{|c|c|c|c|c|c|c|}
\hline & $\Delta \operatorname{Repospr}_{t}$ & $\Delta F G_{t}$ & $\Delta H P W_{t}$ & $\Delta R C s p r_{t}$ & $\Delta D F L_{t}$ & $\Delta D F L f i n_{t}$ \\
\hline & \multicolumn{6}{|c|}{ Panel A: USD market } \\
\hline$\Delta F G_{t}$ & 0.41 & & & & & \\
\hline$\Delta H P W_{t}$ & 0.54 & 0.48 & & & & \\
\hline$\Delta R C \operatorname{spr}_{t}$ & 0.15 & 0.53 & 0.79 & & & \\
\hline$\Delta D F L_{t}$ & 0.41 & 0.30 & 0.33 & 0.21 & & \\
\hline$\Delta D F L$ fin $_{t}$ & 0.41 & 0.26 & 0.29 & 0.14 & 0.99 & \\
\hline \multirow[t]{2}{*}{$\Delta \xi_{t}$} & 0.63 & 0.44 & 0.71 & 0.62 & 0.43 & 0.37 \\
\hline & \multicolumn{6}{|c|}{ Panel B: EUR market } \\
\hline$\Delta F G_{t}$ & 0.42 & & & & & \\
\hline$\Delta H P W_{t}$ & 0.50 & 0.45 & & & & \\
\hline$\Delta R C \operatorname{spr}_{t}$ & 0.10 & 0.51 & 0.76 & & & \\
\hline$\Delta D F L_{t}$ & 0.31 & 0.31 & 0.26 & 0.18 & & \\
\hline$\Delta D F L$ fin $_{t}$ & 0.30 & 0.27 & 0.21 & 0.11 & 0.99 & \\
\hline$\Delta \xi_{t}$ & 0.63 & 0.50 & 0.68 & 0.63 & 0.28 & 0.26 \\
\hline
\end{tabular}

Notes: The table displays pairwise correlations between monthly changes in $\xi_{t}$ and the funding and market liquidity components that are unspanned by default risk. $\xi_{t}$ is estimated using the $\mathbb{A}(2,2,1)$ specification. The unspanned liquidity components are the residuals from regressions of liquidity proxies on the first two principal

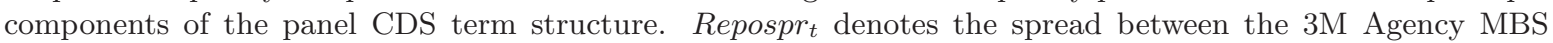
general collateral (GC) repo rate and the $3 \mathrm{M}$ Treasury GC repo rate (in basis points). $F G_{t}$ denotes the Fontaine and Garcia (2011) liquidity factor. $H P W_{t}$ denotes the $\mathrm{Hu}$, Pan, and Wang (2010) liquidity factor. $R C s p r_{t}$ denotes the spread between the $10 \mathrm{Y}$ par yields on Refcorp bonds and off-the-run Treasuries. $D F L_{t}$ and DFL fint denotes the Dick-Nielsen, Feldhutter, and Lando (2012) liquidity factors for the overall corporate bond market and for bonds issued by financial institutions, respectively.

Table 7: Pairwise correlations 


\begin{tabular}{|c|c|c|c|c|c|c|}
\hline$\Delta$ Repospr $_{t}$ & $\Delta F G_{t}$ & $\Delta H P W_{t}$ & $\Delta R C \operatorname{spr}_{t}$ & $\triangle D F L_{t}$ & $\Delta D F L$ fin $_{t}$ & $\operatorname{adj} R^{2}$ \\
\hline \multicolumn{7}{|c|}{ Panel A: USD market } \\
\hline \multirow{6}{*}{$\begin{array}{l}0.039^{* * *} \\
(4.232)\end{array}$} & & & & & & 0.370 \\
\hline & $\begin{array}{l}1.757^{*} \\
(1.852)\end{array}$ & & & & & 0.160 \\
\hline & & $\begin{array}{l}0.556^{* * *} \\
(6.926)\end{array}$ & & & & 0.492 \\
\hline & & & $\begin{array}{l}0.039^{* * *} \\
(3.628)\end{array}$ & & & 0.357 \\
\hline & & & & $\frac{1.092^{* *}}{(2.318)}$ & & 0.150 \\
\hline & & & & & $\begin{array}{l}0.855^{* *} \\
(2.010)\end{array}$ & 0.104 \\
\hline $\begin{array}{l}0.035^{*} \\
(1.907)\end{array}$ & $\begin{array}{l}-0.523 \\
(-0.652)\end{array}$ & $\begin{array}{l}-0.041 \\
(-0.185)\end{array}$ & $\begin{array}{l}0.039^{* * *} \\
(2.763)\end{array}$ & $\begin{array}{l}0.300 \\
(0.980)\end{array}$ & & 0.619 \\
\hline \multirow[t]{2}{*}{$\begin{array}{l}0.034^{\text {*** }} \\
(4.407)\end{array}$} & & & $\begin{array}{l}0.034^{* * *} \\
(4.511)\end{array}$ & & & 0.644 \\
\hline & \multicolumn{6}{|c|}{ Panel B: EUR market } \\
\hline \multirow{6}{*}{$\begin{array}{l}0.025^{* * *} \\
(4.638)\end{array}$} & & & & & & 0.377 \\
\hline & ${ }_{(2.536)}^{1.265^{* *}}$ & & & & & 0.225 \\
\hline & & $\begin{array}{l}0.358^{* * *} \\
(4.419)\end{array}$ & & & & 0.442 \\
\hline & & & $\begin{array}{l}0.026^{* * *} \\
(3.633)\end{array}$ & & & 0.371 \\
\hline & & & & $\begin{array}{l}0.457 \\
(1.476)\end{array}$ & & 0.045 \\
\hline & & & & & $\begin{array}{l}0.371 \\
(1.373)\end{array}$ & 0.030 \\
\hline $\begin{array}{l}0.028^{* * *} \\
(3.579)\end{array}$ & $\begin{array}{l}-0.202 \\
(-0.475)\end{array}$ & $\begin{array}{l}-0.104 \\
(-0.704)\end{array}$ & $\begin{array}{l}0.031^{* * *} \\
(2.614)\end{array}$ & $\begin{array}{l}0.020 \\
(0.174)\end{array}$ & & 0.670 \\
\hline $\begin{array}{l}0.023^{* * *} \\
(5.924)\end{array}$ & & & $\begin{array}{l}0.023^{\text {*** }} \\
(5.918)\end{array}$ & & & 0.699 \\
\hline
\end{tabular}

Notes: The table displays results from regressing monthly changes in $\xi(t)$ on monthly changes in the funding and market liquidity components that are unspanned by default risk. $\xi_{t}$ is estimated using the $\mathbb{A}(2,2,1)$ specification. The unspanned liquidity components are the residuals from regressions of liquidity proxies on the first two principal components of the panel CDS term structure. Repospr $r_{t}$ denotes the spread between the 3M Agency MBS general collateral (GC) repo rate and the 3M Treasury GC repo rate (in basis points). $F G_{t}$ denotes the Fontaine and Garcia (2011) liquidity factor. $H P W_{t}$ denotes the $\mathrm{Hu}$, Pan, and Wang (2010) liquidity factor. $R C s p r_{t}$ denotes the spread between the $10 \mathrm{Y}$ par yields on Refcorp bonds and off-the-run Treasuries. $D F L_{t}$ and DFLfint denotes the Dick-Nielsen, Feldhutter, and Lando (2012) liquidity factors for the overall corporate bond market and for bonds issued by financial institutions, respectively. Intercepts are not reported. T-statistics computed from White (1980) standard errors are in parentheses. *, **, and $* * *$ denote significance at the $10 \%$, $5 \%$, and $1 \%$ levels, respectively.

Table 8: The non-default component and liquidity 


\begin{tabular}{|c|c|c|c|c|c|c|c|}
\hline & \multicolumn{7}{|c|}{ Maturity } \\
\hline & $3 \mathrm{M}$ & $1 \mathrm{Y}$ & $2 \mathrm{Y}$ & $3 \mathrm{Y}$ & $4 \mathrm{Y}$ & $5 \mathrm{Y}$ & $10 Y$ \\
\hline & \multicolumn{7}{|c|}{ Original decomposition: $\mathbb{A}(2,2,1), C D S_{\text {TrMean }}$} \\
\hline Default & $\begin{array}{l}28.1 \\
(26.8)\end{array}$ & $\begin{array}{l}25.2 \\
(17.2)\end{array}$ & $\begin{array}{l}24.0 \\
(12.5)\end{array}$ & $\begin{array}{l}23.8 \\
(10.7)\end{array}$ & $\begin{array}{l}23.9 \\
(9.8)\end{array}$ & $\begin{array}{l}24.1 \\
(9.2)\end{array}$ & ${ }_{(5.8)}^{28.6^{\dagger}}$ \\
\hline \multirow[t]{2}{*}{ Non-default } & $\begin{array}{l}33.4 \\
(45.2)\end{array}$ & $\begin{array}{l}20.4 \\
(27.3)\end{array}$ & $\begin{array}{l}10.6 \\
(14.1)\end{array}$ & $\begin{array}{l}7.2 \\
(9.5)\end{array}$ & $\begin{array}{l}5.5 \\
(7.3)\end{array}$ & $\begin{array}{l}4.5 \\
(6.0)\end{array}$ & ${ }_{(3.5)} 1.8^{\dagger}$ \\
\hline & \multicolumn{7}{|c|}{ Panel $A: \mathbb{A}(2,1,1), C D S_{\text {TrMean }}$} \\
\hline Default & $\begin{array}{l}25.1 \\
(17.2)\end{array}$ & $\begin{array}{l}25.1 \\
(15.3)\end{array}$ & $\begin{array}{l}25.0 \\
(13.2)\end{array}$ & $\begin{array}{l}25.0 \\
(11.5)\end{array}$ & $\begin{array}{l}24.9 \\
(10.2)\end{array}$ & $\begin{array}{l}24.9 \\
(9.0)\end{array}$ & $\begin{array}{l}26.8^{\dagger} \\
(5.2)\end{array}$ \\
\hline \multirow[t]{2}{*}{ Non-default } & $\begin{array}{l}35.8 \\
(45.2)\end{array}$ & $\begin{array}{l}21.3 \\
(26.4)\end{array}$ & $\begin{array}{c}10.9 \\
(13.5)\end{array}$ & $\begin{array}{l}7.4 \\
(9.2)\end{array}$ & $\begin{array}{l}5.7 \\
(7.0)\end{array}$ & $\begin{array}{l}4.6 \\
(5.7)\end{array}$ & ${ }_{(3.4)}^{1.9^{\dagger}}$ \\
\hline & \multicolumn{7}{|c|}{ Panel B: $\mathbb{A}(2,2,2), C D S_{\text {TrMean }}$} \\
\hline Default & $\begin{array}{l}27.8 \\
(25.2)\end{array}$ & $\begin{array}{l}23.3 \\
(15.7)\end{array}$ & $\begin{array}{l}21.5 \\
(11.2)\end{array}$ & $\begin{array}{l}21.0 \\
(9.5)\end{array}$ & $\begin{array}{l}21.1 \\
(8.7)\end{array}$ & $\underset{(8.2)}{21.3}$ & $\underbrace{25.7^{\dagger}}_{(5.2)}$ \\
\hline \multirow[t]{2}{*}{ Non-default } & $\begin{array}{l}31.9 \\
(45.1)\end{array}$ & $\begin{array}{l}22.3 \\
(27.2)\end{array}$ & $\begin{array}{l}13.8 \\
(14.8)\end{array}$ & $\begin{array}{l}10.1 \\
(10.2)\end{array}$ & $\begin{array}{l}7.9 \\
(7.9)\end{array}$ & $\begin{array}{l}6.5 \\
(6.4)\end{array}$ & $\frac{2.9^{\dagger}}{(3.8)^{\dagger}}$ \\
\hline & \multicolumn{7}{|c|}{ Panel $C: \mathbb{A}(2,2,1)$, stochastic $\Lambda(t), C D S_{\text {TrMean }}$} \\
\hline Default & $\begin{array}{l}25.7 \\
(26.8)\end{array}$ & $\begin{array}{c}24.3 \\
(17.8)\end{array}$ & $\begin{array}{l}23.8 \\
(13.1)\end{array}$ & $\begin{array}{l}23.9 \\
(11.2)\end{array}$ & $\begin{array}{l}24.2 \\
(10.2)\end{array}$ & $\begin{array}{c}24.5 \\
(9.5)\end{array}$ & ${ }_{(5.9)}^{29.4^{\dagger}}$ \\
\hline \multirow[t]{2}{*}{ Non-default } & $\begin{array}{r}35.9 \\
(48.2)\end{array}$ & $\begin{array}{l}21.7 \\
(28.9)\end{array}$ & $\begin{array}{l}11.3 \\
(14.9)\end{array}$ & $\begin{array}{c}7.7 \\
(10.1)\end{array}$ & $\begin{array}{l}5.9 \\
(7.7)\end{array}$ & $\begin{array}{l}4.8 \\
(6.3)\end{array}$ & ${ }_{(3.7)^{\dagger}}^{\dagger}$ \\
\hline & \multicolumn{7}{|c|}{ Panel D: $\mathbb{A}(2,2,1)$, no LIBOR, CDS $S_{\text {TrMean }}$} \\
\hline Default & $\begin{array}{l}29.1 \\
(27.3)\end{array}$ & $\begin{array}{l}25.4 \\
(17.2)\end{array}$ & $\begin{array}{l}23.9 \\
(12.5)\end{array}$ & $\begin{array}{l}23.7 \\
(10.7)\end{array}$ & $\begin{array}{l}23.8 \\
(9.8)\end{array}$ & $\begin{array}{l}24.0 \\
(9.2)\end{array}$ & ${ }_{(5.8)}^{28.5^{\dagger}}$ \\
\hline \multirow[t]{2}{*}{ Non-default } & $\begin{array}{l}31.8 \\
(40.9)\end{array}$ & $\begin{array}{l}20.8 \\
(26.6)\end{array}$ & $\begin{array}{c}10.8 \\
(13.8)\end{array}$ & $\begin{array}{l}7.3 \\
(9.3)\end{array}$ & $\begin{array}{l}5.6 \\
(7.1)\end{array}$ & $\begin{array}{l}4.6 \\
(5.8)\end{array}$ & $\begin{array}{l}1.8^{\dagger} \\
(3.3)\end{array}$ \\
\hline & \multicolumn{7}{|c|}{ Panel E: $\mathbb{A}(2,2,1), C D S_{L I Q 1}$} \\
\hline Default & $\begin{array}{l}25.8 \\
(24.9)\end{array}$ & $\begin{array}{l}24.5 \\
(16.9)\end{array}$ & $\begin{array}{l}24.0 \\
(12.6)\end{array}$ & $\begin{array}{l}24.1 \\
(10.9)\end{array}$ & $\begin{array}{l}24.3 \\
(9.9)\end{array}$ & $\begin{array}{l}24.6 \\
(9.3)\end{array}$ & ${ }_{(5.9)}^{29.0^{\dagger}}$ \\
\hline \multirow[t]{2}{*}{ Non-default } & $\begin{array}{l}35.7 \\
(46.2)\end{array}$ & $\begin{array}{l}22.0 \\
(28.0)\end{array}$ & $\begin{array}{l}11.3 \\
(14.4)\end{array}$ & $\begin{array}{l}7.7 \\
(9.7)\end{array}$ & $\begin{array}{l}5.9 \\
(7.4)\end{array}$ & $\begin{array}{l}4.8 \\
(6.1)\end{array}$ & ${ }_{(3.6)}^{1.9^{\dagger}}$ \\
\hline & \multicolumn{7}{|c|}{ Panel F: $\mathbb{A}(2,2,1), C D S_{L I Q 2}$} \\
\hline Default & $\begin{array}{l}31.0 \\
(28.5)\end{array}$ & $\begin{array}{l}26.2 \\
(17.8)\end{array}$ & $\begin{array}{l}24.0 \\
(12.5)\end{array}$ & $\begin{array}{l}23.3 \\
(10.5)\end{array}$ & $\begin{array}{l}23.1 \\
(9.5)\end{array}$ & $\begin{array}{l}23.1 \\
(8.9)\end{array}$ & ${ }_{(5.6)}^{27.1^{\dagger}}$ \\
\hline Non-default & $\begin{array}{l}31.7 \\
(43.4)\end{array}$ & $\begin{array}{l}19.7 \\
(26.8)\end{array}$ & $\begin{array}{l}10.2 \\
(13.8)\end{array}$ & $\begin{array}{l}7.0 \\
(9.4)\end{array}$ & $\begin{array}{l}5.4 \\
(7.2)\end{array}$ & $\begin{array}{l}4.4 \\
(5.9)\end{array}$ & $\begin{array}{c}1.7^{\dagger} \\
(3.4)\end{array}$ \\
\hline
\end{tabular}

Notes: The table shows alternative decompositions, for the USD market, of the spread term structure indexed to $3 \mathrm{M}$ LIBOR, SPREAD $3 M$. Each spread is decomposed into a default and a non-default component and the table displays means and, in parentheses, standard deviations of the two components. The top panel displays the original decomposition (i.e. for the $\mathbb{A}(2,2,1)$ specification combined with the $C D S_{\text {TrMean }}$ measure of interbank default risk.) Panels $\mathrm{A}$ and $\mathrm{B}$ display results for the $\mathbb{A}(2,1,1)$ and $\mathbb{A}(2,2,2)$ specifications, respectively, combined with the $C D S_{T r M e a n}$ measure of interbank default risk. Panel $\mathrm{C}$ displays results for the $\mathbb{A}(2,2,1)$ specification with a stochastic $\Lambda(t)$ combined with the $C D S_{T r M e a n}$ measure of interbank default risk. Panel D displays results for the $\mathbb{A}(2,2,1)$ specification combined with the $C D S_{T r M e a n}$ measure of interbank default risk, but without using LIBOR rates in the estimation. Panels $\mathrm{E}$ and $\mathrm{F}$ display results for the $\mathbb{A}(2,2,1)$ specification combined with the $C D S_{L I Q 1}$ and $C D S_{L I Q 2}$ measures of interbank default risk, respectively. Units are basis points. Each time series consists of 895 daily observations from August 09, 2007 to January 12, 2011, except those marked with $\dagger$ which consist of 643 daily observations from July 28, 2008 to January 12, 2011.

Table 9: Alternative decomposition of the term structure of USD interbank risk 


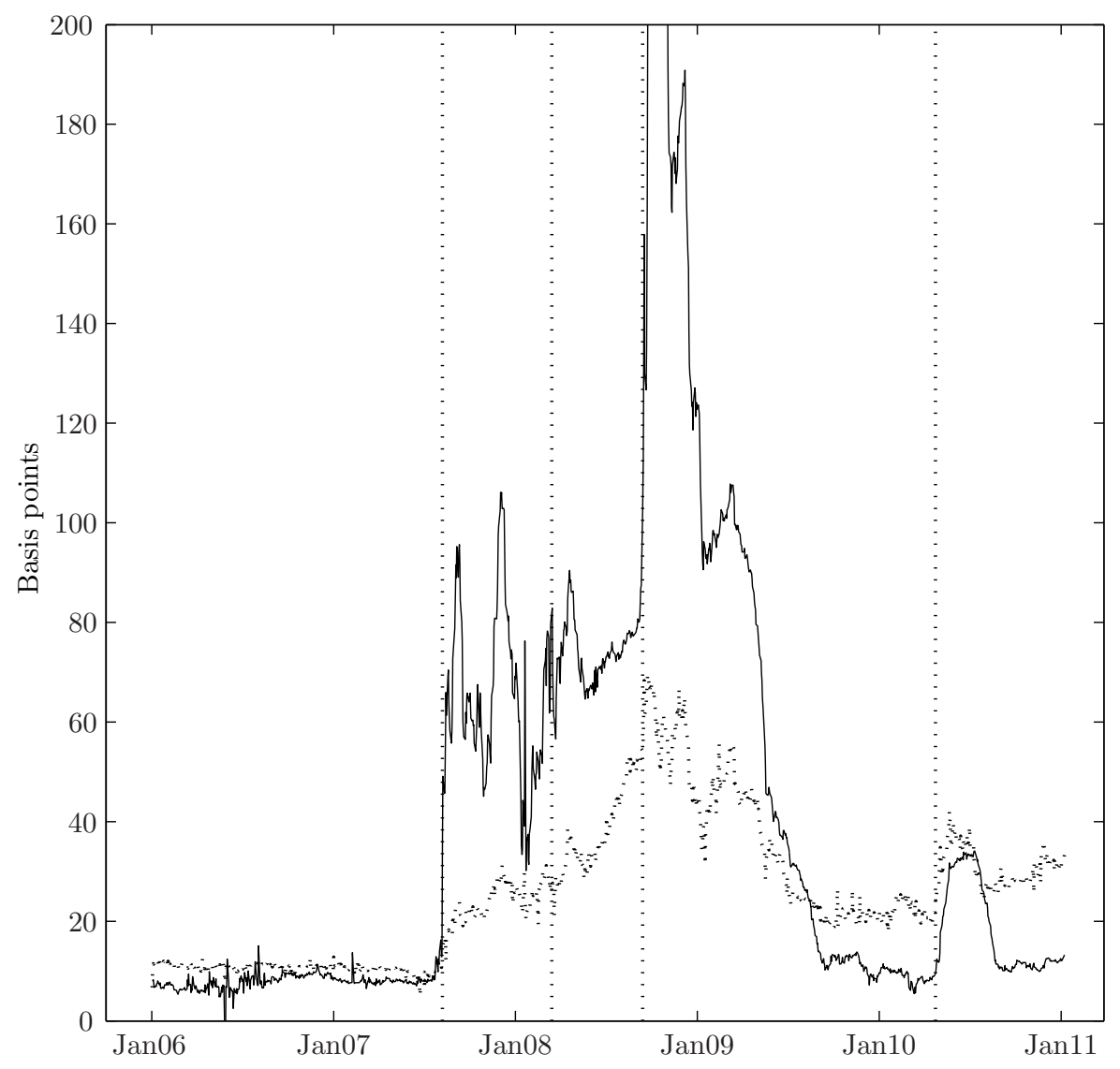

Figure 1: Money market and swap market spreads

The figures shows time-series of the spread between 3M LIBOR and the 3M OIS rate (solid line) and the spread between the rate on a $5 \mathrm{Y}$ interest rate swap indexed to $3 \mathrm{M}$ LIBOR and the $5 \mathrm{Y}$ OIS rate (dotted line). Note that the 3M LIBOR-OIS spread reached a maximum 366 basis points on October 10, 2008. The vertical dotted lines mark the beginning of the financial crisis on August 9, 2007, the sale of Bear Stearns to J.P. Morgan on March 16, 2008, the Lehman Brothers bankruptcy filing on September 15, 2008, and the downgrade of Greece's debt to non-investment grade status by Standard and Poor's on April 27, 2010. Both time series consists of 1313 daily observations from January 02, 2006 to January 12, 2011. 
$\mathbb{A}(2,1,1)$
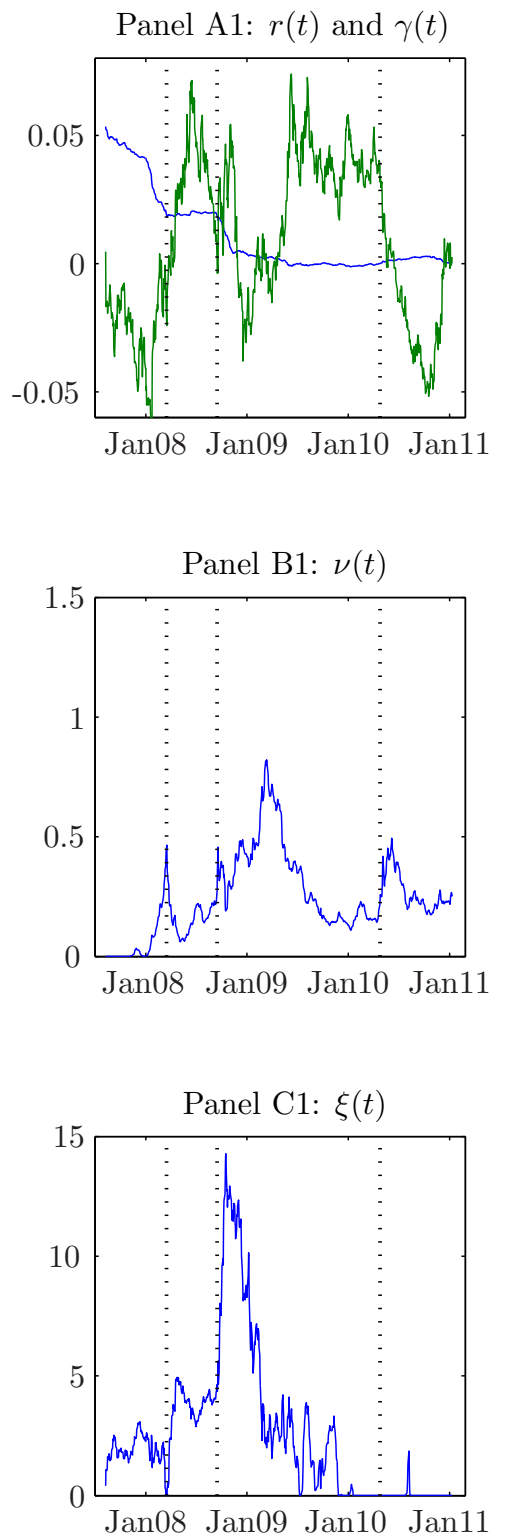

$\mathbb{A}(2,2,1)$
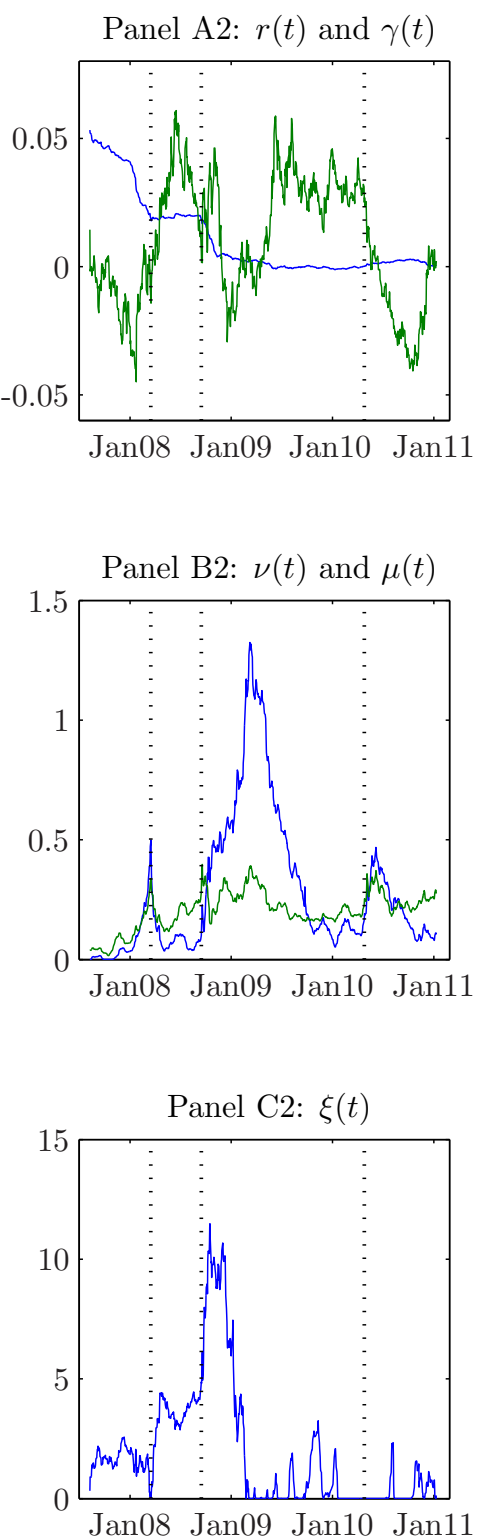

$\mathbb{A}(2,2,2)$

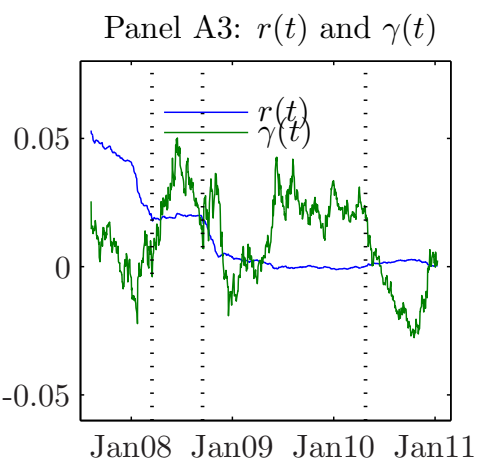

Panel B3: $\nu(t)$ and $\mu(t)$

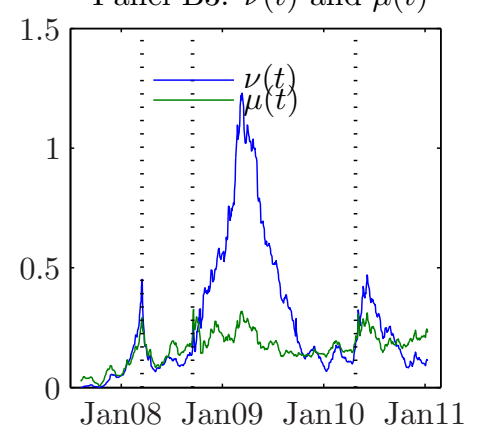

Panel C3: $\xi(t)$ and $\epsilon(t)$

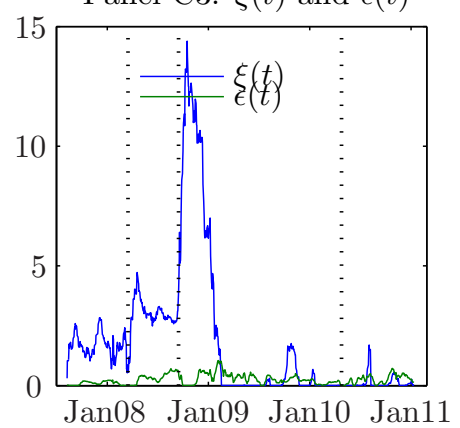

Figure 2: State variables, USD

The figure shows the state variables for the three model specifications estimated on USD data The vertical dotted lines mark the sale of Bear Stearns to J.P. Morgan on March 16, 2008, the Lehman Brothers bankruptcy filing on September 15, 2008, and the downgrade of Greece's debt to non-investment grade status by Standard and Poor's on April 27, 2010. Each time series consists of 895 daily observations from August 09, 2007 to January 12, 2011. 
$\mathbb{A}(2,1,1)$

Panel A1: 3M default prob.

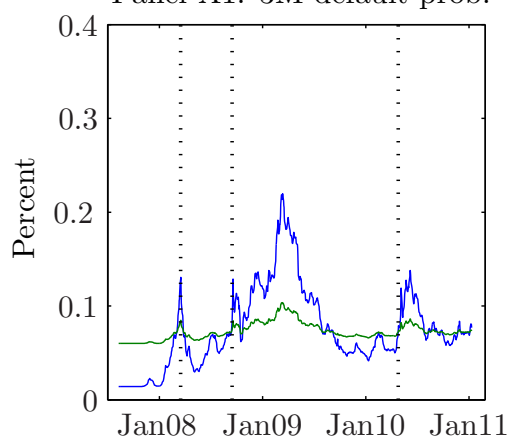

Panel B1: 6M default prob.

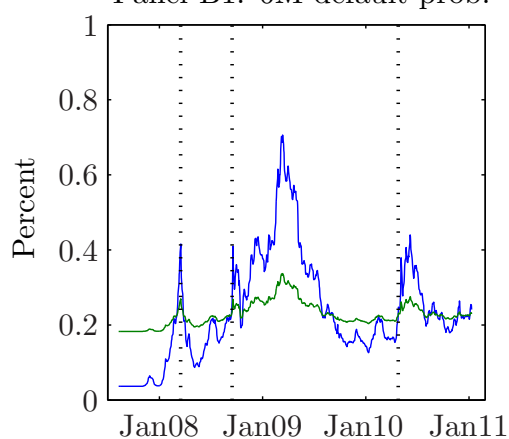

$\mathbb{A}(2,2,1)$

Panel A2: 3M default prob.

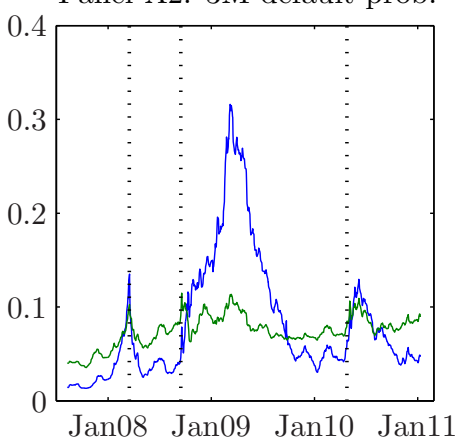

Panel B2: 6M default prob.

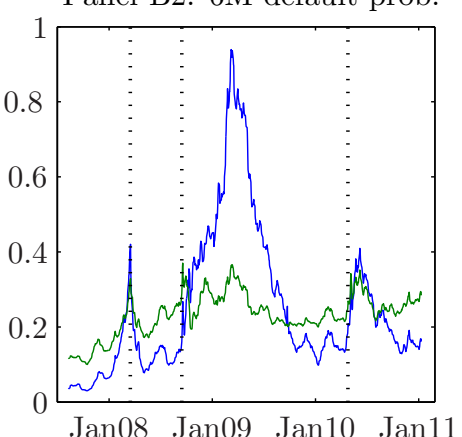

$\mathbb{A}(2,2,2)$

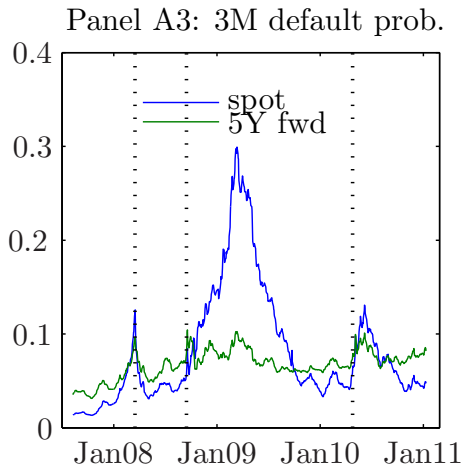

Panel B3: 6M default prob.

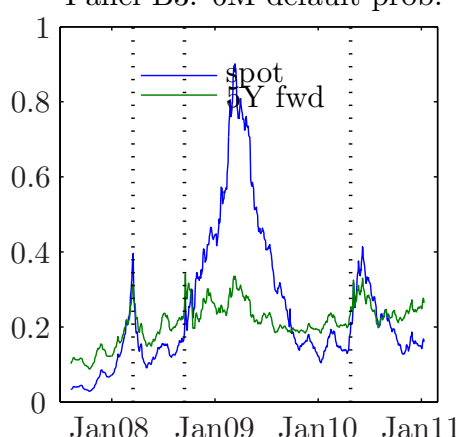

Figure 3: Default probabilities, USD

The top panels display the risk-neutral 3M expected default probability (EDP) for an average bank within the current panel as well as for an average bank within the refreshed panel in five year's time. The bottom panels display the corresponding 6M EDPs. The vertical dotted lines mark the sale of Bear Stearns to J.P. Morgan on March 16, 2008, the Lehman Brothers bankruptcy filing on September 15, 2008, and the downgrade of Greece's debt to non-investment grade status by Standard and Poor's on April 27, 2010. Each time series consists of 895 daily observations from August 09, 2007 to January 12, 2011. 

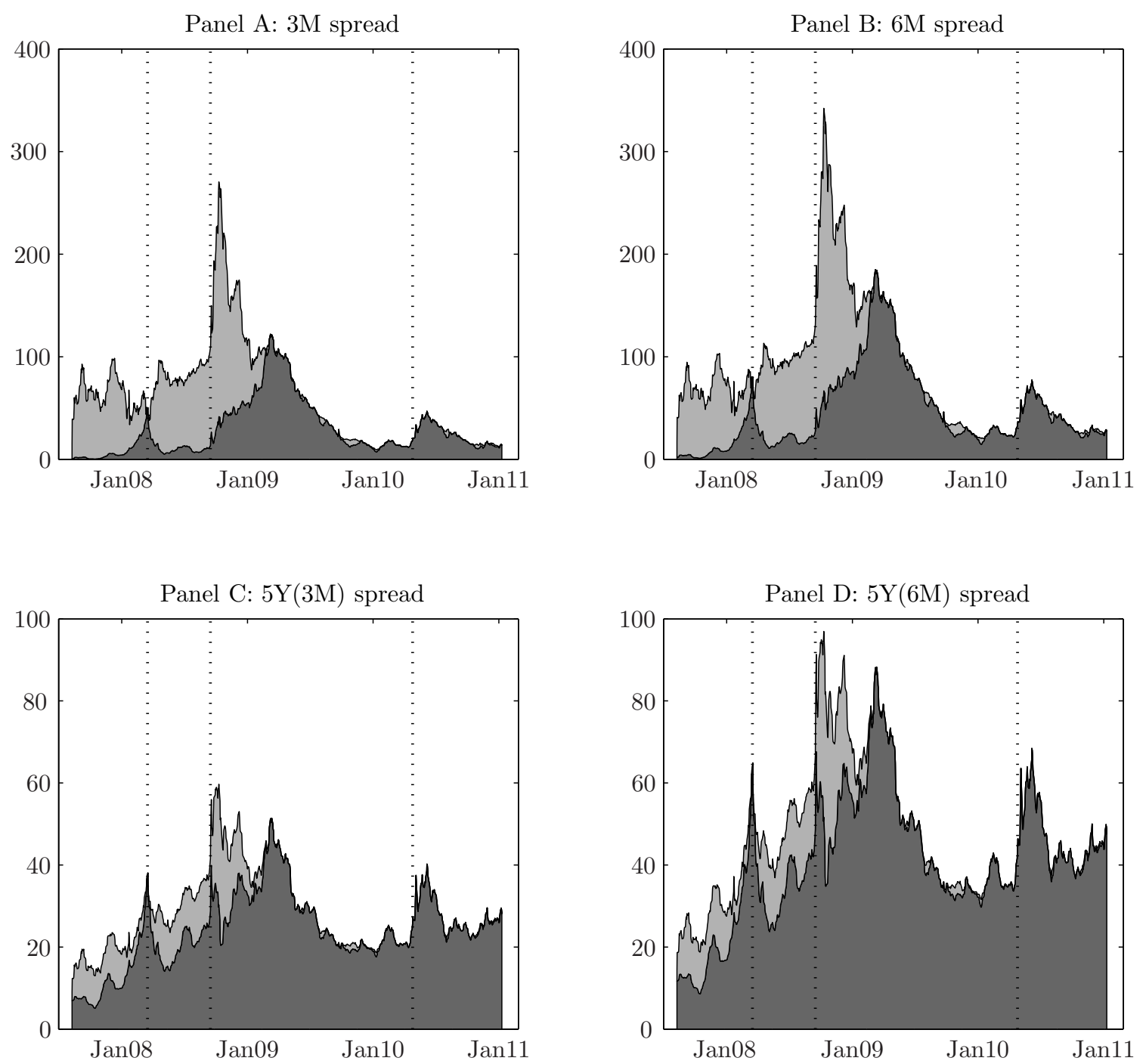

Figure 4: Decomposition of USD interbank risk

Decomposing USD interbank risk into default (dark-grey) and non-default (light-grey) components using the $\mathbb{A}(2,2,1)$ specification and the $C D S_{T r M e a n}$ measure of interbank default risk. Panels $\mathrm{A}$ and B display decompositions of the $3 \mathrm{M}$ and $6 \mathrm{M}$ LIBOR-OIS spread, respectively. Panels $\mathrm{C}$ and D display decompositions of the $5 Y$ IRS-OIS spread indexed to $3 \mathrm{M}$ and $6 \mathrm{M}$ LIBOR, respectively. Units are basis points. The vertical dotted lines mark the sale of Bear Stearns to J.P. Morgan on March 16, 2008, the Lehman Brothers bankruptcy filing on September 15, 2008, and the downgrade of Greece's debt to non-investment grade status by Standard and Poor's on April 27, 2010. Each time series consists of 895 daily observations from August 09, 2007 to January $12,2011$. 

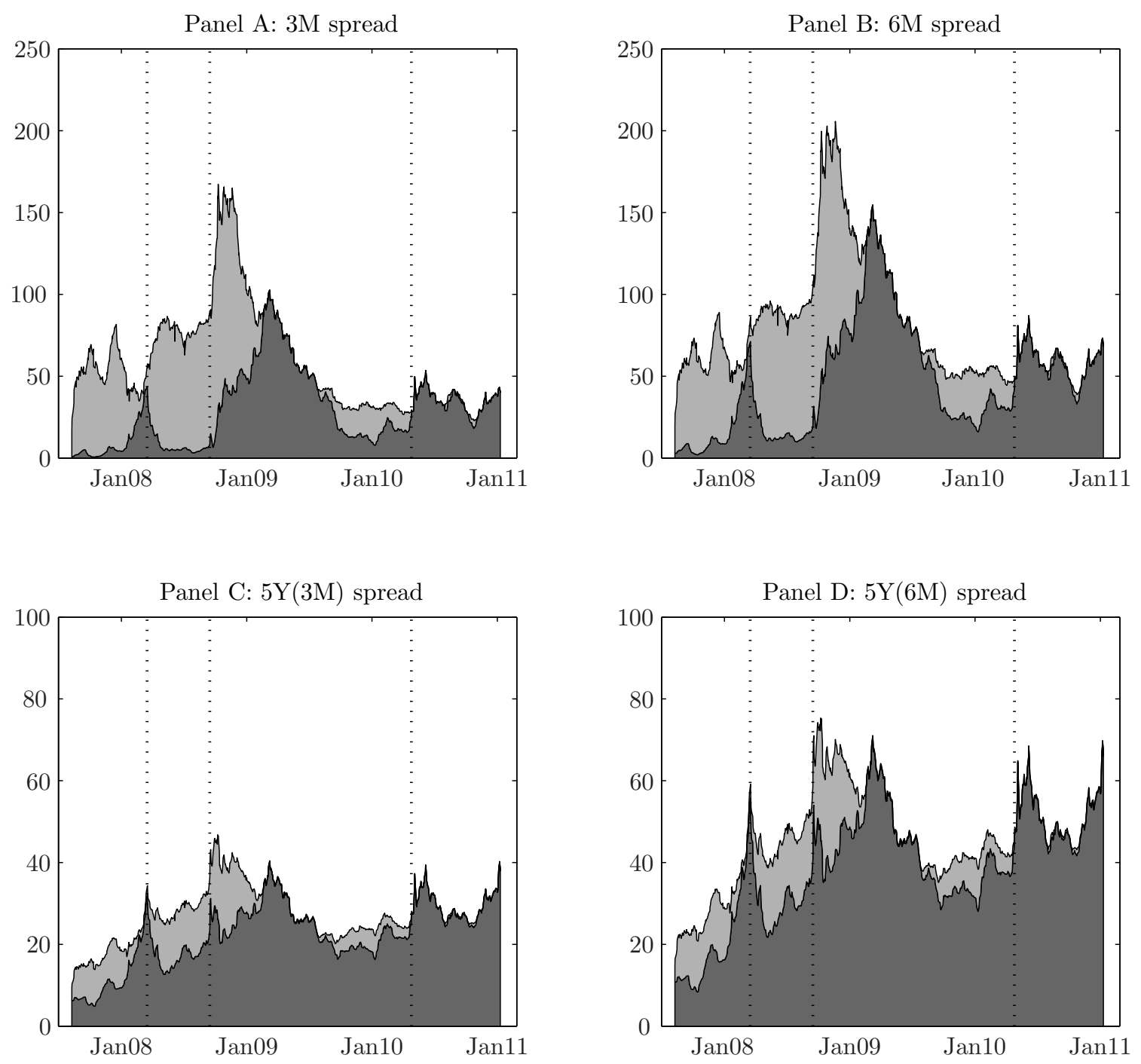

Figure 5: Decomposition of EUR interbank risk

Decomposing EUR interbank risk into default (dark-grey) and non-default (light-grey) components using the $\mathbb{A}(2,2,1)$ specification and the $C D S_{\text {Median }}$ measure of interbank default risk. Panels A and B display decompositions of the $3 \mathrm{M}$ and $6 \mathrm{M}$ EURIBOR-OIS spread, respectively. Panels $\mathrm{C}$ and D display decompositions of the $5 Y$ IRS-OIS spread indexed to $3 \mathrm{M}$ and $6 \mathrm{M}$ EURIBOR, respectively. Units are basis points. The vertical dotted lines mark the sale of Bear Stearns to J.P. Morgan on March 16, 2008, the Lehman Brothers bankruptcy filing on September 15, 2008, and the downgrade of Greece's debt to non-investment grade status by Standard and Poor's on April 27, 2010. Each time series consists of 895 daily observations from August 09, 2007 to January $12,2011$. 

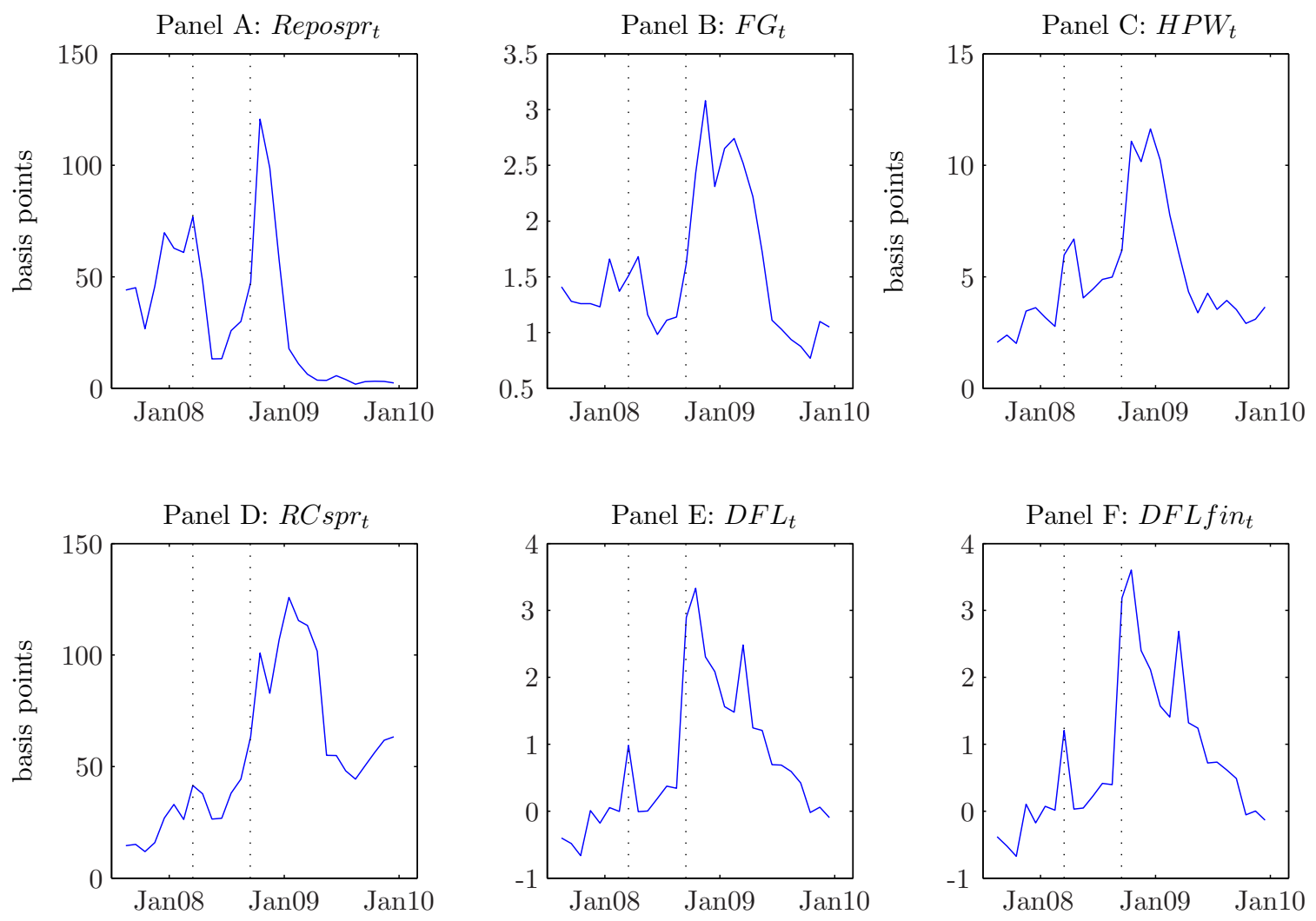

Figure 6: Liquidity proxies

Repospr $_{t}$ denotes the spread between the 3M Agency MBS general collateral (GC) repo rate and the 3M Treasury GC repo rate (in basis points). $F G_{t}$ denotes the Fontaine and Garcia (2011) liquidity factor. $H P W_{t}$ denotes the $\mathrm{Hu}$, Pan, and Wang (2010) liquidity factor. RCspr denotes the spread between the $10 \mathrm{Y}$ par

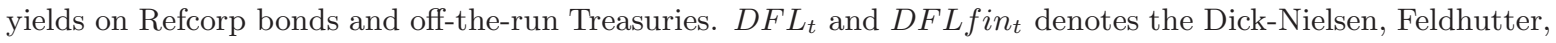
and Lando (2012) liquidity factors for the overall corporate bond market and for bonds issued by financial institutions, respectively. The vertical dotted lines mark the sale of Bear Stearns to J.P. Morgan on March 16, 2008, and the Lehman Brothers bankruptcy filing on September 15, 2008. Each time series consists of 27 monthly observations from August, 2007 to December, 2010. 


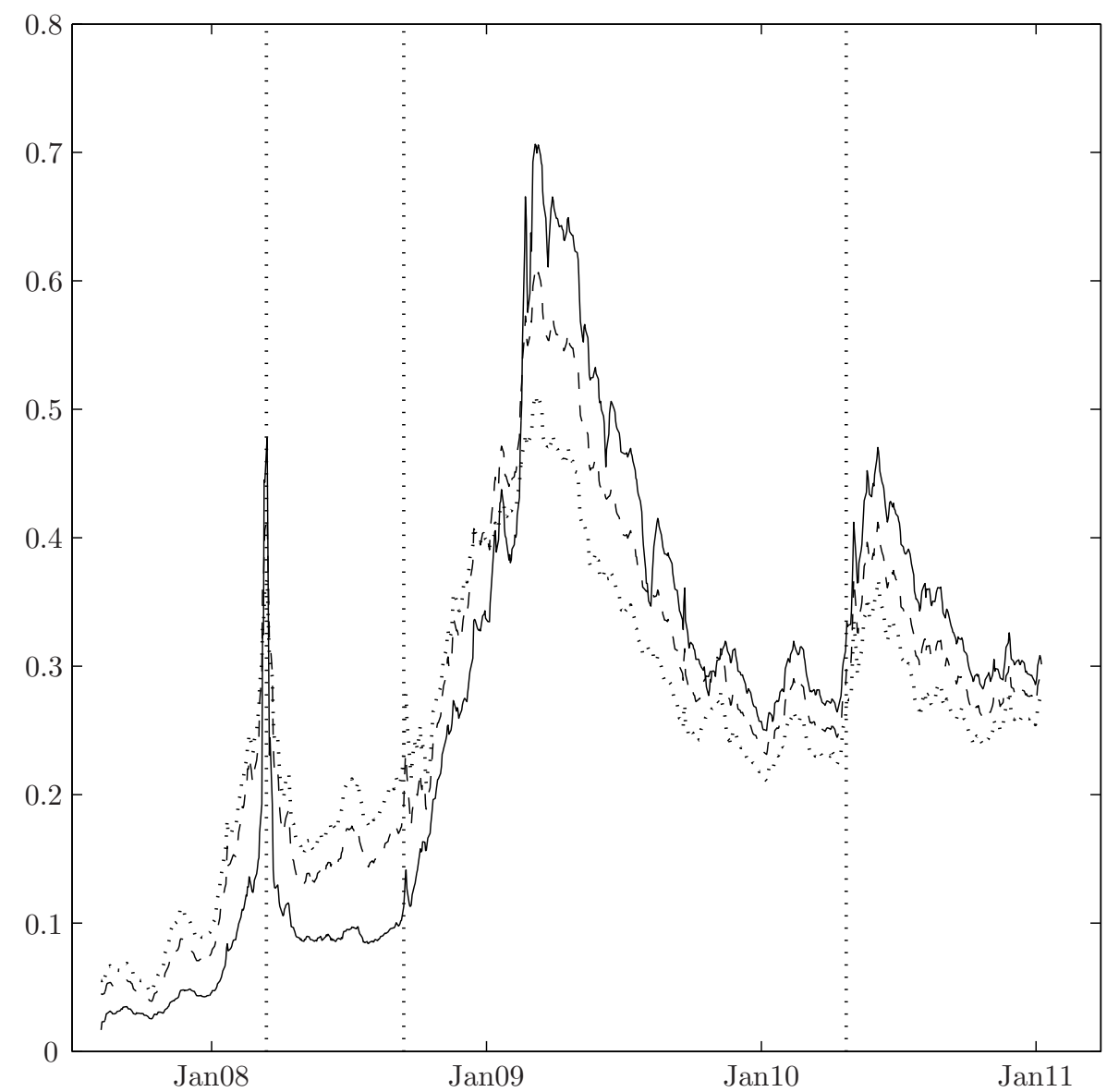

Figure 7: Pricing of interbank risk in the swap market

The figure displays the model-implied instantaneous Sharpe ratios on a swap spread strategy consisting of receiving the fixed rate in an IRS indexed to $3 \mathrm{M}$ LIBOR and paying the fixed rate in an OIS of the same maturity. The solid, dashed, and dotted lines correspond to the $1 \mathrm{Y}, 5 \mathrm{Y}$, and $10 \mathrm{Y}$ swap maturities, respectively. The Sharpe ratios are computed for the $\mathbb{A}(2,2,1)$ specification estimated on the USD market. The vertical dotted lines mark the sale of Bear Stearns to J.P. Morgan on March 16, 2008, the Lehman Brothers bankruptcy filing on September 15, 2008, and the downgrade of Greece's debt to non-investment grade status by Standard and Poor's on April 27, 2010. Each time series consists of 895 daily observations from August 09, 2007 to January 12, 2011. 


\section{References}

Acharya, V., D. Gromb, and T. Yorulmazer (2007): "Imperfect competition in the interbank market for liquidity as a rationale for central banking," Working paper, NYU, forthcoming American Economic Journal: Macroeconomics.

Acharya, V., H. Shin, And T. Yorulmazer (2010): "Crisis resolution and bank liquidity," Working paper, NYU, forthcoming Review of Financial Studies.

ACharya, V. AND D. Skeie (2010): "A model of liquidity hoarding and term premia in inter-bank markets," Working paper, NYU and Federal Reserve Bank of New York, forthcoming Journal of Monetary Economics.

Afonso, G., A. Kovner, And A. Schoar (2009): "Stressed, not frozen: The Federal Funds market in the financial crisis," Working paper, Federal Reserve Bank of New York and MIT, forthcoming Journal of Finance.

Allen, F., E. Carletti, and D. Gale (2009): "Interbank market liquidity and central bank intervention," Journal of Monetary Economics, 56:639-652.

Andersen, L. And V. Piterbarg (2010): Interest Rate Modeling, Atlantic Financial Press.

Ang, A. And F. Longstaff (2011): "Systemic sovereign credit risk: Lessons from the U.S. and Europe," Working paper, UCLA and Columbia Business School.

Angelini, P., A. Nobili, And M. Picillo (2009): "The interbank market after August 2007: what has changed and why?," Working paper, Bank of Italy.

Arora, N., P. Gandhi, And F. Longstaff (2009): "Counterparty credit risk and the credit default swap market," Working paper, UCLA.

Bartolini, L., S. Hilton, S. Sundaresan, And C. Tonetti (2011): "Collateral values by asset class: Evidence from primary securities dealers," Review of Financial Studies, 24:248-278.

Beber, A., M. Brandt, And K. Kavajecz (2009): "Flight-to-quality or flight-to-liquidity? Evidence from the Euro-area bond market," Review of Financial Studies, 22:925-957.

Bianchetti, M. (2009): "Two curves, one price. Pricing \& hedging interest rate derivatives decoupling forwarding and discounting yield curves," Working paper, Banca Intesa San Paolo.

Blanco, R., S. Brennan, And I. Marsh (2005): "An empirical analysis of the dynamic relation between investment-grade bonds and credit default swaps," Journal of Finance, 60:2255-2281.

Bongaerts, D., F. De Jong, And J. Driessen (2011): "Derivative pricing with liquidity risk: Theory and evidence from the credit default swap market," Journal of Finance, 66:203-240. 
Brunnermeier, M. K. And L. H. Pedersen (2009): "Market liquidity and funding liquidity," Review of Financial Studies, 22:2201-2238.

Buhler, W. And M. Trapp (2010): "Time-varying credit risk and liquidity premia in bond and CDS markets," Working paper, University of Cologne.

Christoffersen, P., K. Jakobs, L. Karoui, and K. Mimouni (2009): "Non-linear filtering in affine term structure models: Evidence from the term structure of swap rates," Working paper, McGill University.

Collin-Dufresne, P. And B. Solnik (2001): "On the term structure of default premia in the swap and LIBOR markets," Journal of Finance, 56:1095-1116.

Copeland, A., A. Martin, And M. Walker (2010): "The tri-party repo market before the 2010 reforms," Working paper, Federal Reserve Bank of New York.

Dai, Q. And K. Singleton (2002): "Expectations puzzles, time-varying risk premia, and affine models of the term structure," Journal of Financial Economics, 63:415-442.

Diamond, D. W. And R. G. Rajan (2010): "Fear of fire sales, illiquidity seeking, and credit freezes," Working paper, University of Chicago, forthcoming Quarterly Journal of Economics.

Dick-Nielsen, J., P. Feldhutter, And D. Lando (2012): "Corporate bond liquidity before and after the onset of the subprime crisis," Journal of Financial Economics, 103:471-492.

Duan, J.-C. And J.-G. Simonato (1999): "Estimating and testing exponential-affine term structure models by Kalman filter," Review of Quantitative Finance and Accounting, 13:111-135.

Duarte, J., F. Longstaff, And F. Yu (2007): "Risk and return in fixed income arbitrage: Nickels in front of a steamroller," Review of Financial Studies, 20:769-811.

Duffee, G. (2002): "Term premia and interest rate forecasts in affine models," Journal of Finance, $57: 405-443$.

Duffie, D. (1996): "Special repo rates," Journal of Finance, 51:493-526.

Duffie, D., D. Filipović, and W. Schachermayer (2003): "Affine processes and applications in finance," Annals of Applied Probability, 13:984-1053.

Duffie, D. And M. Huang (1996): "Swap rates and credit quality," Journal of Finance, 51:921-950.

Duffie, D. And K. Singleton (1997): "An econometric model of the term structure of interest rate swap yields," Journal of Finance, 52:1287-1323.

(1999): "Modeling term structures of defaultable bonds," Review of Financial Studies, 12:687-720.

(2003): Credit Risk: Pricing, Measurement, and Management, Princeton University Press. 
EISENSChMidt, J. AND J. TAPKING (2009): "Liquidity risk premia in unsecured interbank money markets," Working paper, European Central Bank.

Feldhutter, P. And D. Lando (2008): "Decomposing swap spreads," Journal of Financial Economics, 88:375-405.

Filipović, D. (2009): Term Structure Models - A Graduate Course, Springer.

Fontaine, J.-S. And R. Garcia (2011): "Bond liquidity premia," Working paper, Bank of Canada and EDHEC Business School, forthcoming Review of Financial Studies.

FujiI, M., Y. Shimada, And A. Takahashi (2009): "A market model of interest rates with dynamic spreads in the presence of collateral and multiple currencies," Working paper, Shinsei Bank and University of Tokyo.

Gale, D. And T. Yorulmazer (2011): "Liquidity hoarding," Working paper, NYU and Federal Reserve Bank of New York.

Gyntelberg, J. And P. Wooldridge (2008): "Interbank rate fixings during the recent turmoil," BIS Quarterly Review, March:59-72.

Henrard, M. (2009): "The irony in the derivatives discounting part II. The crisis," Working paper, Dexia Bank.

Hordahl, P. And M. King (2008): "Developments in repo markets during the financial turmoil," BIS Quarterly Review, December:37-53.

Hu, X., J. PAN, AND J. WANG (2010): "Noise as information for illiquidity," Working paper, MIT.

IMF (2008): Global Financial Stability Report (October),

ISDA (2010): Margin Survey, 2010,

Jarrow, R., D. LANDo, AND F. Yu (2005): "Default risk and diversification: Theory and empirical implications," Mathematical Finance, 15:1-26.

Johannes, M. AND S. Sundaresan (2007): "The impact of collateralization on swap rates," Journal of Finance, 62:383-410.

Jordan, B. AND S. Jordan (1997): "Special repo rates: An empirical analysis," Journal of Finance, 52:2051-2072.

Krishnamurthy, A., S. Nagel, And D. Orlov (2011): "Sizing up repo," Working paper, Northwestern University and Stanford University.

Lando, D. (2004): Credit Risk Modeling: Theory and Applications, Princeton University Press.

Liu, J., F. Longstaff, And R. Mandell (2006): "The market price of risk in interest rate swaps: The roles of default and liquidity risks," Journal of Business, 79:2337-2359. 
Longstaff, F. (2000): "The term structure of very short-term rates: New evidence for the expectations hypothesis," Journal of Financial Economics, 58:397-415.

(2004): "The flight-to-liquidity premium in U.S. Treasury bond prices," Journal of Business, 77:511-526.

Longstaff, F., S. Mithal, And E. Neis (2005): "Corporate yield spreads: Default risk or liquidity? New evidence from the credit default swap market," Journal of Finance, 60:2213-2253.

McAndrews, J., A. Sarkar, and Z. Wang (2008): "The effect of the term auction facility on the London Inter-Bank Offered Rate," Working paper, Federal Reserve Bank of New York.

Mercurio, F. (2009): "Interest rates and the credit crunch. New formulas and market models," Working paper, Bloomberg.

(2010): "LIBOR market models with stochastic basis," Working paper, Bloomberg.

Michaud, F.-L. And C. Upper (2008): "What drives interbank rates? Evidence from the Libor panel," BIS Quarterly Review, March:47-58.

Newey, W. And K. West (1987): "A simple, positive semi-definit, heteroscedasticity and autocorrelation consistent covariance matrix," Econometrica, 55:703-708.

Piterbarg, V. (2010): "Funding beyond discounting: Collateral agreements and derivatives pricing," RISK magazine, pages 97-102.

Schwartz, K. (2010): "Mind the gap: disentangling credit and liquidity in risk spreads," Working paper, The Wharton School, University of Pennsylvania.

Smith, J. (2010): "The term structure of money market spreads during the financial crisis," Working paper, New York University.

TAYlor, J. B. And J. C. Williams (2009): "A black swan in the money market," American Economic Journal, Macroeconomics, 1:58-83.

White, H. (1980): "A heteroskedasticity-consistent covariance matrix estimator and a direct test for heteroskedasticity," Econometrica, 48:817-838.

Whittall, C. (2010): "LCH.Clearnet re-values USD 218 trillion swap portfolio using OIS," RISK, June.

Yu, F. (2002): "Modeling expected return on defaultable bonds," Journal of Fixed income, 12:69-81. 


\section{Online Appendix to "The Term Structure of Interbank Risk"}

Damir Filipović and Anders B. Trolle

Ecole Polytechnique Fédérale de Lausanne and Swiss Finance Institute 


\section{E Proof of Lemma C.4}

For the proof of Lemma C.4 we first recall a fundamental comparison result for ordinary differential equations, which is a special case of a more general theorem proved by Volkmann (1972):

Lemma E.1. Let $R(\tau, v)$ be a continuous real map on $\mathbb{R}_{+} \times \mathbb{R}$ and locally Lipschitz continuous in $v$. Let $p(\tau)$ and $q(\tau)$ be differentiable functions satisfying

$$
\begin{aligned}
\partial_{\tau} p(\tau) & \leq R(\tau, p(\tau)) \\
\partial_{\tau} q(\tau) & =R(\tau, q(\tau)) \\
p(0) & \leq q(0) .
\end{aligned}
$$

Then we have $p(\tau) \leq q(\tau)$ for all $\tau \geq 0$.

We only prove the first part of Lemma C.4, the proof of the second part being similar. ${ }^{61}$ It follows from Duffie, Filipović, and Schachermayer (2003, Theorem 2.16), see also Filipović (2009, Theorem 10.3), that the affine transform formula

$$
E^{Q}\left[\exp \left[u_{\nu} \nu(\tau)+u_{\mu} \mu(\tau)\right]\right]=\exp \left[\phi(\tau, u)+\psi_{\nu}(\tau, u) \nu(0)+\psi_{\mu}(\tau, u) \mu(0)\right]
$$

holds, and the expectation on the left hand side is finite in particular, for $u=\left(u_{\nu}, u_{\mu}\right)^{\top} \in \mathbb{R}^{2}$ if $\phi(\tau, u), \psi_{\nu}(\tau, u)$ and $\psi_{\mu}(\tau, u)$ are finite solutions of the corresponding system of Riccati equations

$$
\begin{aligned}
\partial_{\tau} \phi(\tau, u) & =\kappa_{\mu} \theta_{\mu} \psi_{\mu}(\tau, u)+\Lambda \\
\phi(0, u) & =0 \\
\partial_{\tau} \psi_{\nu}(\tau, u) & =\frac{\sigma_{\nu}^{2}}{2} \psi_{\nu}(\tau, u)^{2}-\kappa_{\nu} \psi_{\nu}(\tau, u) \\
\psi_{\nu}(0, u) & =u_{\nu} \\
\partial_{\tau} \psi_{\mu}(\tau, u) & =\frac{\sigma_{\mu}^{2}}{2} \psi_{\mu}(\tau, u)^{2}-\kappa_{\mu} \psi_{\mu}(\tau, u)+\kappa_{\nu} \psi_{\nu}(\tau, u) \\
\psi_{\mu}(0, u) & =u_{\mu} .
\end{aligned}
$$

It is thus enough to show that both, $\psi_{\nu}\left(\tau, u^{*}\right)$ and $\psi_{\mu}\left(\tau, u^{*}\right)$, are finite for all $\tau \geq 0$ and for $u^{*}:=\left(-D_{\nu}\left(T-t_{0}\right),-D_{\mu}\left(T-t_{0}\right)\right)^{\top}$.

\footnotetext{
${ }^{61}$ Indeed, after replacing $\frac{1}{\zeta_{\lambda} \kappa_{\lambda}+1}$ by 1 in (E.2), and in the definition of $p_{\nu}(\tau), \Theta_{\nu}$ and $C_{\nu}$ below, the proof of the second part of Lemma C.4 is literally the same.
} 
We first provide a bound for $u^{*}$. Note that $D_{\lambda}(\tau)$, defined as solution of (43), is given by $D_{\lambda}(\tau)=-\frac{1}{\kappa_{\lambda}}\left(1-e^{-\kappa_{\lambda} \tau}\right)$. This implies $-\frac{1}{\kappa_{\lambda}} \leq D_{\lambda}(\tau) \leq 0$ and thus

$$
-\frac{1}{\zeta_{\lambda} \kappa_{\lambda}+1} \leq \frac{D_{\lambda}(\tau)}{\zeta_{\lambda}-D_{\lambda}(\tau)} \leq 0
$$

In view of (43) and Lemma E.1 we conclude that $p_{\nu}(\tau) \leq D_{\nu}(\tau) \leq 0$ where $p_{\nu}(\tau)$ solves the Riccati differential equation

$$
\begin{aligned}
\partial_{\tau} p_{\nu}(\tau) & =\frac{\sigma_{\nu}^{2}}{2} p_{\nu}(\tau)^{2}-\kappa_{\nu} p_{\nu}(\tau)-\frac{1}{\zeta_{\lambda} \kappa_{\lambda}+1} \\
p_{\nu}(0) & =0
\end{aligned}
$$

The explicit solution of (E.2) is well known to be

$$
p_{\nu}(\tau)=-\frac{\frac{2}{\zeta_{\lambda} \kappa_{\lambda}+1}\left(e^{\Theta_{\nu} \tau}-1\right)}{\Theta_{\nu}\left(e^{\Theta_{\nu} \tau}+1\right)+\kappa_{\nu}\left(e^{\Theta_{\nu} \tau}-1\right)}
$$

where $\Theta_{\nu}$ is defined in (47), see e.g. Filipović (2009, Lemma 10.12). We thus obtain the estimate

$$
0 \leq-D_{\nu}\left(T-t_{0}\right) \leq-p_{\nu}\left(T-t_{0}\right)=C_{\nu}
$$

Similarly, in view of (43), (E.3) and Lemma E.1, we infer that $p_{\mu}(\tau) \leq D_{\mu}(\tau) \leq 0$ where $p_{\mu}(\tau)$ solves the Riccati equation

$$
\begin{aligned}
\partial_{\tau} p_{\mu}(\tau) & =\frac{\sigma_{\mu}^{2}}{2} p_{\mu}(\tau)^{2}-\kappa_{\mu} p_{\mu}(\tau)-\kappa_{\nu} C_{\nu} \\
p_{\mu}(0) & =0 .
\end{aligned}
$$

Again, the explicit solution of (E.4) is readily available, see e.g. Filipović (2009, Lemma 10.12):

$$
p_{\mu}(\tau)=-\frac{2 \kappa_{\nu} C_{\nu}\left(e^{\Theta_{\mu} \tau}-1\right)}{\Theta_{\mu}\left(e^{\Theta_{\mu} \tau}+1\right)+\kappa_{\mu}\left(e^{\Theta_{\mu} \tau}-1\right)}
$$

where $\Theta_{\mu}$ is defined in (48). Moreover, it follows by inspection that $p_{\mu}(\tau) \downarrow P_{1}$ as $\tau \rightarrow \infty$ for the left critical point

$$
P_{1}=\frac{\kappa_{\mu}-\sqrt{\kappa_{\mu}^{2}+2 \sigma_{\mu}^{2} \kappa_{\nu} C_{\nu}}}{\sigma_{\mu}^{2}}
$$

of the differential equation (E.4), and we obtain the estimates

$$
0 \leq-D_{\mu}\left(T-t_{0}\right) \leq-p_{\mu}\left(T-t_{0}\right)=C_{\mu} \leq-P_{1} .
$$


Next, we give a priori bounds on $\psi_{\nu}\left(\tau, u^{*}\right)$ and $\psi_{\mu}\left(\tau, u^{*}\right)$. Denote by $P_{2}=\frac{2 \kappa_{\nu}}{\sigma_{\nu}^{2}}$ the right critical point of the homogeneous Riccati differential equation (E.1) for $\psi_{\nu}(\tau, u)$, and denote by

$$
q_{\nu}(\tau)=\frac{2 \kappa_{\nu} C_{\nu}}{\left(2 \kappa_{\nu}-\sigma_{\nu}^{2} C_{\nu}\right) e^{\kappa_{\nu} \tau}+\sigma_{\nu}^{2} C_{\nu}}
$$

the solution of (E.1) for $\psi_{\nu}(\tau, u)$ with initial condition $u_{\nu}=C_{\nu}$, see e.g. Filipović (2009, Lemma 10.12). It then follows from Lemma E.1 and by inspection that

$$
0 \leq \psi_{\nu}\left(\tau, u^{*}\right) \leq q_{\nu}(\tau) \text { and } q_{\nu}(\tau) \downarrow 0 \text { for } \tau \rightarrow \infty \text { if } C_{\nu}<P_{2},
$$

which is (49).

Now suppose that (49) holds, that is, $C_{\nu}<P_{2}$. Combining (E.7) with (E.1), (E.5) and Lemma E.1 implies

$$
0 \leq \psi_{\mu}\left(\tau, u^{*}\right) \leq q_{\mu}(\tau)
$$

where $q_{\mu}(\tau)$ solves the time-inhomogeneous Riccati equation

$$
\begin{aligned}
\partial_{\tau} q_{\mu}(\tau) & =\frac{\sigma_{\mu}^{2}}{2} q_{\mu}(\tau)^{2}-\kappa_{\mu} q_{\mu}(\tau)+\kappa_{\nu} q_{\nu}(\tau) \\
q_{\mu}(0) & =C_{\mu} .
\end{aligned}
$$

If $\sigma_{\mu}=0$ then obviously $q_{\mu}(\tau)$ is finite for all $\tau \geq 0$, and there is nothing left to prove. So from now on we assume that $\sigma_{\mu}>0$ and $\kappa_{\mu} \geq 0$. Since there is no closed form expression for $q_{\mu}(\tau)$ available in general, we are going to control $q_{\mu}$ from above by a time-inhomogeneous linear differential equation. Hereto note the elementary fact that

$$
\frac{\sigma_{\mu}^{2}}{2} x^{2}-\kappa_{\mu} x+\kappa_{\nu} q_{\nu}(\tau) \leq-\frac{\kappa_{\mu}}{2} x+\kappa_{\nu} q_{\nu}(\tau) \text { for all } 0 \leq x \leq \frac{\kappa_{\mu}}{\sigma_{\mu}^{2}}
$$

Hence, by Lemma E.1, the solution $f$ of

$$
\begin{aligned}
\partial_{\tau} f(\tau) & =-\frac{\kappa_{\mu}}{2} f(\tau)+\kappa_{\nu} q_{\nu}(\tau) \\
f(0) & =C_{\mu}
\end{aligned}
$$

dominates $q_{\mu}$, that is, $0 \leq q_{\mu}(\tau) \leq f(\tau)$ for all $\tau \geq 0$, if

$$
f(\tau) \leq \frac{\kappa_{\mu}}{\sigma_{\mu}^{2}}
$$

for all $\tau \geq 0$. 
We now claim that (E.8) holds for any fixed $\tau \geq 0$ if and only if

$$
\int_{0}^{\tau} e^{\frac{\kappa_{\mu}}{2} s}\left(\kappa_{\nu} q_{\nu}(s)-\frac{\kappa_{\mu}^{2}}{2 \sigma_{\mu}^{2}}\right) d s \leq \frac{\kappa_{\mu}}{\sigma_{\mu}^{2}}-C_{\mu} .
$$

Indeed, $f$ can be represented by the variation of constants formula

$$
f(\tau)=e^{-\frac{\kappa_{\mu}}{2} \tau} C_{\mu}+\int_{0}^{\tau} e^{-\frac{\kappa_{\mu}}{2}(\tau-s)} \kappa_{\nu} q_{\nu}(s) d s .
$$

Hence (E.8) is equivalent to

$$
C_{\mu}+\int_{0}^{\tau} e^{\frac{\kappa \mu}{2} s} \kappa_{\nu} q_{\nu}(s) d s \leq \frac{\kappa_{\mu}}{\sigma_{\mu}^{2}} e^{\frac{\kappa \mu}{2} \tau}
$$

The right hand side of (E.11) can be rewritten as

$$
\frac{\kappa_{\mu}}{\sigma_{\mu}^{2}} e^{\frac{\kappa \mu}{2} \tau}=\frac{\kappa_{\mu}}{\sigma_{\mu}^{2}}\left(e^{\frac{\kappa \mu}{2} \tau}-1\right)+e^{\frac{\kappa \mu}{2} \tau}=\frac{\kappa_{\mu}^{2}}{2 \sigma_{\mu}^{2}} \int_{0}^{\tau} e^{\frac{\kappa \mu}{2} s} d s+\frac{\kappa_{\mu}}{\sigma_{\mu}^{2}}
$$

Plugging this in (E.11) and rearrange terms yields (E.9).

In view of (E.7) we infer that the maximum of the left hand side of (E.9) is attained at $\tau=\tau^{*}$ where

$$
\tau^{*}=\inf \left\{\tau \geq 0 \mid \kappa_{\mu}^{2}-2 \sigma_{\mu}^{2} \kappa_{\nu} q_{\nu}(\tau) \geq 0\right\}<\infty,
$$

and which by (E.6) can be written as in (51). Hence the bound (E.8) holds for all $\tau \geq 0$ if and only if (E.8) holds for $\tau=\tau^{*}$. This again is equivalent to (50), since the integral in (E.10) can be expressed as ${ }^{62}$

$$
\begin{aligned}
\int_{0}^{\tau} e^{-\frac{\kappa_{\mu}}{2}(\tau-s)} \kappa_{\nu} q_{\nu}(s) d s=\frac{4 \kappa_{\nu}^{2}}{\kappa_{\mu} \sigma_{\nu}^{2}}\left({ } _ { 2 } F _ { 1 } \left(1, \frac{\kappa_{\mu}}{2 \kappa_{\nu}}\right.\right. & \left.; \frac{\kappa_{\mu}+2 \kappa_{\nu}}{2 \kappa_{\nu}} ; \frac{\left(\sigma_{\nu}^{2} C_{\nu}-2 \kappa_{\nu}\right) e^{\kappa_{\nu} \tau}}{\sigma_{\nu}^{2} C_{\nu}}\right) \\
& \left.-e^{-\frac{\kappa_{\mu}}{2} \tau}{ }_{2} F_{1}\left(1, \frac{\kappa_{\mu}}{2 \kappa_{\nu}} ; \frac{\kappa_{\mu}+2 \kappa_{\nu}}{2 \kappa_{\nu}} ; \frac{\sigma_{\nu}^{2} C_{\nu}-2 \kappa_{\nu}}{\sigma_{\nu}^{2} C_{\nu}}\right)\right)
\end{aligned}
$$

where ${ }_{2} F_{1}$ is the Gauss hypergeometric function. Finally, note that $\tau^{*}=0$ if and only if $\frac{\kappa_{\mu}^{2}}{2 \kappa_{\nu} \sigma_{\mu}^{2}} \geq C_{\nu}$. In this case, (E.5) implies

$$
\sigma_{\mu}^{2} C_{\mu} \leq \sqrt{\kappa_{\mu}^{2}+2 \sigma_{\mu}^{2} \kappa_{\nu} C_{\nu}}-\kappa_{\mu} \leq \sqrt{2 \kappa_{\mu}^{2}}-\kappa_{\mu} \leq \kappa_{\mu},
$$

so that (50) automatically holds. This finishes the proof of Lemma C.4.

\footnotetext{
${ }^{62}$ We obtained the integral formula from the computational software program Mathematica.
} 


\section{References}

Duffie, D., D. Filipović, And W. Schachermayer (2003): "Affine processes and applications in finance," Annals of Applied Probability, 13:984-1053.

Filipović, D. (2009): Term Structure Models - A Graduate Course, Springer.

Volkmann, P. (1972): "Gewöhnliche Differentialungleichungen mit quasimonoton wachsenden Funktionen in topologischen Vektorräumen," Mathematische Zeitschrift, 127:157-164. 


\section{F On quotation conventions for basis swaps}

As discussed in Section 2.4, there is no universally accepted quotation convention for basis swaps. The two most common conventions are the following. In the first convention (I), and the one we use in the paper, the cash flow in a basis swap is the difference between the cash flows in two IRS indexed to different floating rates. In the case of a $3 \mathrm{M} / 6 \mathrm{M}$ basis swap and with $\delta=3 \mathrm{M}$, this implies that one party pays $\delta L(t-\delta, t)$ quarterly, while the other party pays $2 \delta L(t-2 \delta, t)$ semi-annually, with the fixed spread payments made semi-annually in the USD market and annually in the EUR market. Given that our model has an analytical solution to an IRS, it also has an analytical solution to a basis swap defined according to this convention.

In the second convention (II), all payments occur at the frequency of the longer floating rate with the shorter floating rate paid compounded. In the case of a $3 \mathrm{M} / 6 \mathrm{M}$ basis swap, this implies for both markets that on a semi-annual basis one party pays $\delta L(t-2 \delta, t-\delta)(1+$ $\delta L(t-\delta, t))+\delta L(t-\delta, t)$ plus a fixed spread, while the other party pays $2 \delta L(t-2 \delta, t)$. If we assume that payments are made on tenor structure (5) with $t_{i}=t_{i-1}+2 \delta$, the basis swap rate according to this convention is given by

$$
\begin{aligned}
B S_{\delta, 2 \delta}(t, T)= & \frac{1}{\sum_{i=1}^{N} 2 \delta P_{c}\left(t, t_{i}\right)}\left(\sum _ { i = 1 } ^ { N } E _ { t } ^ { Q } \left[e ^ { - \int _ { t } ^ { t _ { i } } r _ { c } ( s ) d s } \left(2 \delta L\left(t_{i-1}, t_{i}\right)-\right.\right.\right. \\
& \left.\left.\left.\left(\delta L\left(t_{i-1}, t_{i-1}+\delta\right)\left(1+\delta L\left(t_{i-1}+\delta, t_{i}\right)\right)+\delta L\left(t_{i-1}+\delta, t_{i}\right)\right)\right)\right]\right) .
\end{aligned}
$$

We now quantify the difference between the two market conventions. For a given parameter set and state vector, we compute $3 \mathrm{M} / 6 \mathrm{M}$ basis swap rates implied by conventions (I) analytically, and by convention (II) via simulation. ${ }^{63}$ We consider both markets and for each market two state vectors: the mean state vector and the state vector on the day of the widest $1 Y$ basis swap rate. Table F.1 shows the spread term structures implied by convention (I). It also shows the differences between the spread term structures implied by convention (II) and (I) along with the standard errors of the simulated basis swap rates in parentheses. On a typical day, the differences between the spreads implied by the two conventions are very small both in absolute and relative terms. Even on the day of the widest $1 Y$ basis swap rate, the differences between the spreads remain very small in relative terms.

\footnotetext{
${ }^{63}$ In principle, one can compute basis swap rates for convention (II) analytically as well. However, the expressions are fairly involved, and as the spreads can be simulated very accurately using a low number of simulations, we opt for this approach.
} 


\begin{tabular}{|c|c|c|c|c|c|c|c|}
\hline & \multicolumn{7}{|c|}{ Maturity } \\
\hline & $1 \mathrm{Y}$ & $2 \mathrm{Y}$ & $3 \mathrm{Y}$ & $4 \mathrm{Y}$ & $5 \mathrm{Y}$ & $7 \mathrm{Y}$ & $10 \mathrm{Y}$ \\
\hline & \multicolumn{7}{|c|}{ Panel A: USD market, typical day } \\
\hline (I) & 22.087 & 15.449 & 12.525 & 10.864 & 9.791 & 8.496 & 7.482 \\
\hline \multirow[t]{2}{*}{ (II)-(I) } & $\begin{array}{l}-0.125 \\
(0.003)\end{array}$ & $\begin{array}{c}-0.118 \\
(0.004)\end{array}$ & $\begin{array}{c}-0.121 \\
(0.005)\end{array}$ & $\begin{array}{c}-0.126 \\
(0.006)\end{array}$ & $\begin{array}{c}-0.131 \\
(0.007)\end{array}$ & $\begin{array}{c}-0.143 \\
(0.007)\end{array}$ & $\begin{array}{c}-0.168 \\
(0.008)\end{array}$ \\
\hline & \multicolumn{7}{|c|}{ Panel B: USD market, day of widest $1 Y$ spread } \\
\hline (I) & 39.629 & 23.089 & 16.881 & 13.865 & 12.114 & 10.179 & 8.759 \\
\hline \multirow[t]{2}{*}{ (II)-(I) } & $\begin{array}{l}-0.601 \\
(0.008)\end{array}$ & $\begin{array}{c}-0.347 \\
(0.005)\end{array}$ & $\begin{array}{c}-0.265 \\
(0.005)\end{array}$ & $\begin{array}{c}-0.233 \\
(0.005)\end{array}$ & $\begin{array}{c}-0.217 \\
(0.006)\end{array}$ & $\begin{array}{c}-0.208 \\
(0.006)\end{array}$ & $\begin{array}{c}-0.219 \\
(0.008)\end{array}$ \\
\hline & \multicolumn{7}{|c|}{ Panel C: EUR market, typical day } \\
\hline (I) & 14.546 & 12.793 & 11.862 & 11.211 & 10.681 & 9.788 & 8.687 \\
\hline \multirow[t]{2}{*}{ (II)-(I) } & $\begin{array}{l}-0.215 \\
(0.002)\end{array}$ & $\begin{array}{c}-0.202 \\
(0.003)\end{array}$ & $\begin{array}{c}-0.203 \\
(0.004)\end{array}$ & $\begin{array}{c}-0.207 \\
(0.005)\end{array}$ & $\begin{array}{c}-0.209 \\
(0.005)\end{array}$ & $\begin{array}{c}-0.213 \\
(0.007)\end{array}$ & $\begin{array}{c}-0.210 \\
(0.008)\end{array}$ \\
\hline & \multicolumn{7}{|c|}{ Panel D: EUR market, day of widest $1 Y$ spread } \\
\hline (I) & 36.655 & 26.557 & 22.032 & 19.435 & 17.684 & 15.310 & 12.996 \\
\hline (II)-(I) & $\begin{array}{c}-0.836 \\
(0.004) \\
\end{array}$ & $\begin{array}{c}-0.583 \\
(0.005) \\
\end{array}$ & $\begin{array}{c}-0.482 \\
(0.006) \\
\end{array}$ & $\begin{array}{c}-0.431 \\
(0.007) \\
\end{array}$ & $\begin{array}{c}-0.400 \\
(0.008) \\
\end{array}$ & $\begin{array}{c}-0.361 \\
(0.010) \\
\end{array}$ & $\begin{array}{l}-0.322 \\
(0.011) \\
\end{array}$ \\
\hline $\begin{array}{l}\text { Notes: In tl } \\
\text { (I) and (II) } \\
\text { simulation. } \\
\text { by conventi } \\
\text { swap rates } \\
\text { swap rates } \\
\text { in parenthe } \\
\text { using the st } \\
2008 \text { in EU }\end{array}$ & $\begin{array}{l}\text { as a very } \\
\text { d by con } \\
\text { d by con } \\
\text { anels A . } \\
\text { ctor on t } \\
\text { e use the }\end{array}$ & $\begin{array}{l}\text { ient contr } \\
\text { ion (I). Th } \\
\text { ions (II) at } \\
\text { C show re } \\
\text { lay of the } \\
2,2,1 \text { ) spec }\end{array}$ & $\begin{array}{l}\text { variate. In } \\
\text { second line } \\
\text { (I), with st } \\
\text { dest } 1 Y \text { bas bas } \\
\text { dest bas }\end{array}$ & $\begin{array}{l}\text { panel, th } \\
\text { ws the dif } \\
\text { ard errors } \\
\text { ean state } \\
\text { wap rate }\end{array}$ & $\begin{array}{l}\text { ap rates } \\
\text { del, wh } \\
\text { hetic) ar } \\
\text { t line sh } \\
\text { ce betwe } \\
\text { he simul } \\
\text { or, while } \\
\text { ober } 14,\end{array}$ & $\begin{array}{l}\text { d by ma } \\
\text { e latter } \\
\text { basis sy } \\
\text { he term s } \\
\text { e term st } \\
\text { asis swa } \\
\text { ls B and } \\
\text { in USD }\end{array}$ & $\begin{array}{l}\text { convention } \\
\text { mputed b } \\
\text { ate implie } \\
\text { ure of basi } \\
\text { ure of basi } \\
\text { es reporte } \\
\text { how result } \\
\text { October } 13\end{array}$ \\
\hline
\end{tabular}

Table F.1: Impact of differences in market convention for basis swaps 


\section{G On the (lack of) identification of $\zeta_{\lambda}$}

Here, we show that it is very difficult to separately identify $\zeta_{\lambda}$ (where $\frac{1}{\zeta_{\lambda}}$ is the mean jump size in the default intensity) and the process for $\nu(t)$ (the intensity of credit quality deterioration). We consider the $\mathbb{A}(2,2,1)$ specification, but the results also hold true for the other specifications. Let $\bar{\nu}(t)=\frac{1}{\zeta_{\lambda}} \nu(t)$ denote the mean rate of deterioration in the credit quality of an average panel bank. Its dynamics are given by

$$
\begin{aligned}
& d \bar{\nu}(t)=\kappa_{\nu}(\bar{\mu}(t)-\bar{\nu}(t)) d t+\sigma_{\bar{\nu}} \sqrt{\bar{\nu}(t)} d W_{\nu}(t) \\
& d \bar{\mu}(t)=\kappa_{\mu}\left(\theta_{\bar{\mu}}-\bar{\mu}(t)\right) d t+\sigma_{\bar{\mu}} \sqrt{\bar{\mu}(t)} d W_{\mu}(t),
\end{aligned}
$$

where $\bar{\mu}(t)=\frac{1}{\zeta_{\lambda}} \mu(t), \theta_{\bar{\mu}}=\frac{1}{\zeta_{\lambda}} \theta_{\mu}, \sigma_{\bar{\mu}}=\frac{1}{\sqrt{\zeta_{\lambda}}} \sigma_{\mu}$, and $\sigma_{\bar{\nu}}=\frac{1}{\sqrt{\zeta_{\lambda}}} \sigma_{\nu}$.

We price interest rate spreads and CDS spreads for different values of $\zeta_{\lambda}$ keeping the level of $\bar{\nu}(t)$ and its process unchanged. That is, variation in $\zeta_{\lambda}$ is accompanied by adjustments to $\nu(t), \mu(t), \theta_{\mu}, \sigma_{\mu}$, and $\sigma_{\nu}$. We use the parameters reported in Table 4 for the USD market and assume that the state vector equals its sample average. We then vary $\zeta_{\lambda}$ between 10 and 1000 corresponding to mean jump sizes between $1000 \mathrm{bp}$ and $10 \mathrm{bp}$.

Figure G.1 shows interest rate and CDS spreads for the $1 \mathrm{Y}$ and $10 \mathrm{Y}$ maturities as a function of $\frac{1}{\zeta_{\lambda}}$. Overall, the spreads are quite insensitive to the mean jump size, although it appears that combinations of high mean jump sizes and low intensity of credit quality deterioration produce slightly lower spreads than combinations of low jump sizes and high intensity of credit quality deterioration. ${ }^{64}$ This illustrate that, for valuation of spreads, it is the mean rate of deterioration in credit quality that matters, and that it would be very difficult to disentangle $\zeta_{\lambda}$ and the process for $\nu(t)$.

\footnotetext{
${ }^{64} \mathrm{~A}$ similar conclusion was reached by Collin-Dufresne and Solnik (2001), albeit within a simpler model, in their study of spreads between nonrefreshed and refreshed credit quality corporate-bond yields (see their Figure 2).
} 

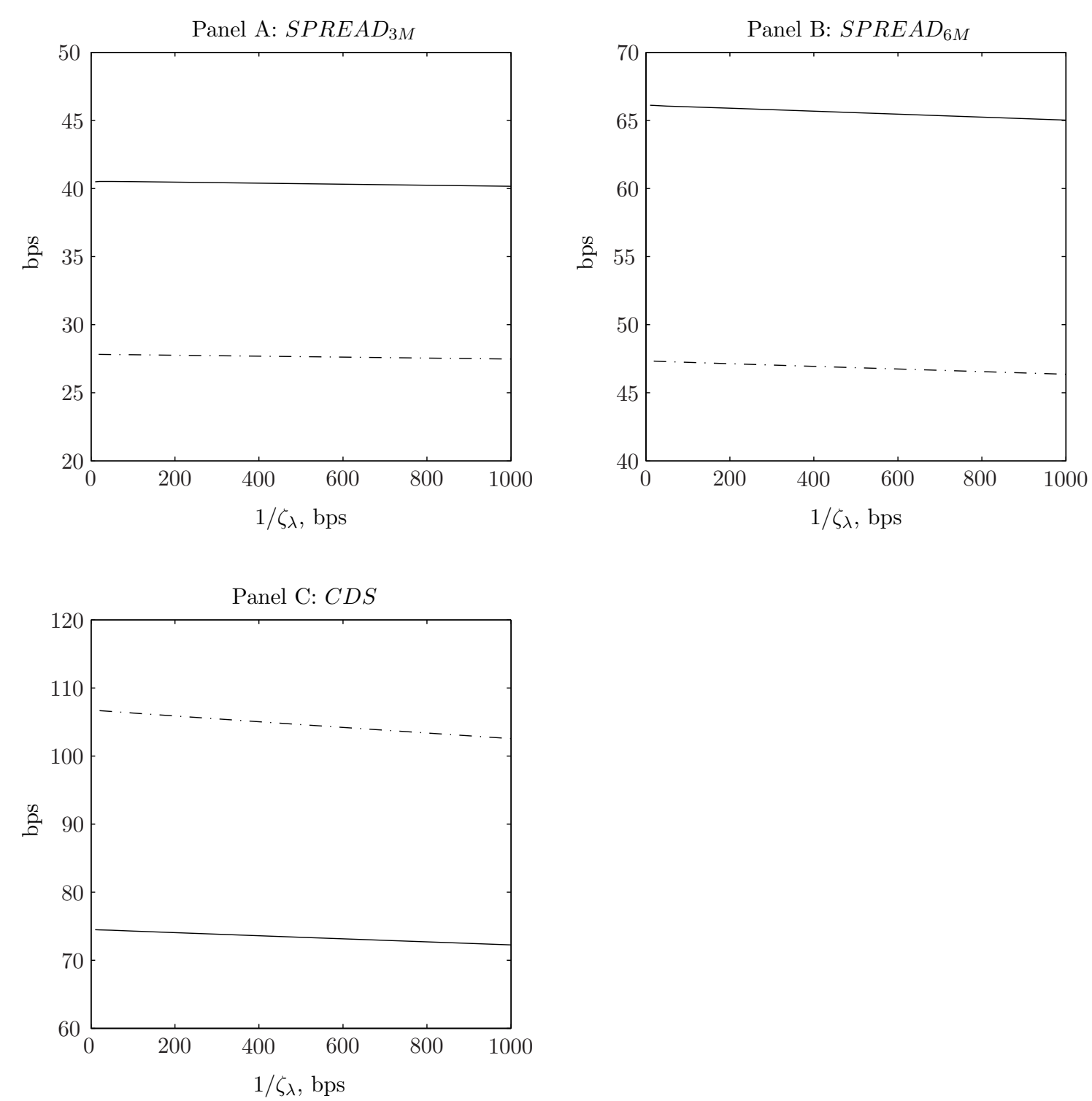

Figure G.1: Sensitivity of spreads to $\frac{1}{\zeta_{\lambda}}$

Sensitivity of interest rate spreads and CDS spreads to variation in the mean jump in the default intensity, $\frac{1}{\zeta_{\lambda}}$, keeping the process for the mean rate of deterioration in credit quality, $\bar{\nu}(t)$, unchanged. $S P R E A D_{3 M}$ denotes the difference between the fixed rates on an IRS indexed to 3M LIBOR and an OIS with the same maturity. $S P R E A D_{6 M}$ denotes the difference between the fixed rates on an IRS indexed to 6M LIBOR and an OIS with the same maturity. Solid lines correspond to the $1 \mathrm{Y}$ maturity while dash-dotted lines correspond to the $10 \mathrm{Y}$ maturity. 


\section{H Additional tables and figures}

Individual RMSEs Tables H.1 and H.2 show individual root mean squared pricing errors (RMSEs) in the USD and EUR markets, respectively, thus complementing Table 5 in the paper.

OIS and repo rates Figure H.1 displays 1W OIS and repo rates, while Figure H.2 displays spreads between 1W OIS and repo rates.

State variables and default probabilities, EUR Figures H.3 and H.4 display the state variables and the default probabilities, respectively, for the three model specifications estimated on EUR data. They are the EUR counterparts to Figures 2 and 3 in the paper.

Time series of alternative decompositions Figures H.5 and H.6 show time series of the alternative decompositions of USD interbank risk at the short end and long end of the term structure, respectively. They complement Table 9 in the paper. 


\begin{tabular}{|c|c|c|c|c|c|c|c|c|c|}
\hline & \multicolumn{9}{|c|}{ Maturity } \\
\hline & $3 \mathrm{M}$ & $6 \mathrm{M}$ & $1 \mathrm{Y}$ & $2 Y$ & $3 \mathrm{Y}$ & $4 \mathrm{Y}$ & $5 \mathrm{Y}$ & $7 \mathrm{Y}$ & $10 \mathrm{Y}$ \\
\hline & \multicolumn{9}{|c|}{ Panel $A: \mathbb{A}(2,1,1)$} \\
\hline$O I S$ & 7.42 & 5.61 & 7.83 & 7.72 & 7.69 & 5.42 & 6.09 & & 11.16 \\
\hline$S P R E A D_{3 M}$ & 12.13 & & 8.96 & 10.67 & 8.90 & 7.23 & 6.22 & & 5.47 \\
\hline$S P R E A D_{6 M}$ & & 10.53 & 7.98 & 5.50 & 6.72 & 8.09 & 9.94 & & 12.56 \\
\hline \multirow[t]{2}{*}{$C D S$} & & 23.09 & 9.73 & 9.25 & 9.23 & 8.15 & 9.95 & 12.05 & 15.52 \\
\hline & \multicolumn{9}{|c|}{ Panel B: $\mathbb{A}(2,2,1)$} \\
\hline OIS & 7.27 & 5.29 & 7.51 & 7.68 & 7.66 & 5.24 & 6.15 & & 11.09 \\
\hline$S P R E A D_{3 M}$ & 11.40 & & 9.67 & 9.90 & 9.81 & 7.65 & 5.86 & & 4.75 \\
\hline$S P R E A D_{6 M}$ & & 8.68 & 8.49 & 5.39 & 4.91 & 5.93 & 8.02 & & 11.76 \\
\hline \multirow[t]{2}{*}{$C D S$} & & 13.28 & 5.52 & 7.00 & 5.95 & 3.90 & 5.68 & 5.66 & 6.28 \\
\hline & \multicolumn{9}{|c|}{ Panel $C: \mathbb{A}(2,2,2)$} \\
\hline$O I S$ & 7.36 & 5.20 & 7.50 & 7.64 & 7.58 & 5.13 & 5.96 & & 10.98 \\
\hline$S P R E A D_{3 M}$ & 10.25 & & 9.84 & 9.10 & 8.37 & 7.65 & 6.07 & & 4.07 \\
\hline$S P R E A D_{6 M}$ & & 10.44 & 7.79 & 3.72 & 3.84 & 5.11 & 7.00 & & 9.88 \\
\hline$C D S$ & & 14.27 & 4.47 & 6.22 & 5.29 & 3.35 & 5.16 & 4.34 & 4.54 \\
\hline
\end{tabular}

Notes: The table reports root mean squared pricing errors (RMSEs) for each point on the term structures of OIS rates, interest rate spreads, and CDS spreads. SPREAD ${ }_{3 M}$ denotes the difference between the fixed rates on an IRS indexed to $3 \mathrm{M}$ LIBOR and an OIS with the same maturity. $S P R E A D_{6 M}$ denotes the difference between the fixed rates on an IRS indexed to $6 \mathrm{M}$ LIBOR and an OIS with the same maturity. Units are basis points.

Table H.1: Individual RMSEs, USD market 


\begin{tabular}{|c|c|c|c|c|c|c|c|c|c|}
\hline & \multicolumn{9}{|c|}{ Maturity } \\
\hline & $3 \mathrm{M}$ & $6 \mathrm{M}$ & $1 \mathrm{Y}$ & $2 \mathrm{Y}$ & $3 \mathrm{Y}$ & $4 \mathrm{Y}$ & $5 \mathrm{Y}$ & $7 \mathrm{Y}$ & $10 \mathrm{Y}$ \\
\hline & \multicolumn{9}{|c|}{ Panel $A: \mathbb{A}(2,1,1)$} \\
\hline$O I S$ & 8.02 & 3.88 & 10.20 & 9.74 & 5.72 & 4.10 & 3.98 & 5.48 & 9.08 \\
\hline$S P R E A D_{3 M}$ & 11.37 & & 8.26 & 9.26 & 9.26 & 8.93 & 8.09 & 7.07 & 6.10 \\
\hline$S P R E A D_{6 M}$ & & 9.81 & 7.94 & 7.10 & 8.04 & 9.14 & 9.32 & 9.91 & 10.90 \\
\hline \multirow[t]{2}{*}{$C D S$} & & 24.35 & 11.15 & 9.75 & 8.39 & 6.48 & 9.31 & 11.17 & 14.96 \\
\hline & \multicolumn{9}{|c|}{ Panel $B: \mathbb{A}(2,2,1)$} \\
\hline OIS & 8.43 & 3.55 & 9.76 & 9.46 & 5.49 & 3.76 & 3.60 & 5.22 & 8.75 \\
\hline$S P R E A D_{3 M}$ & 8.49 & & 7.76 & 9.81 & 10.11 & 9.62 & 8.64 & 7.23 & 5.75 \\
\hline$S P R E A D_{6 M}$ & & 9.23 & 7.10 & 3.93 & 4.44 & 5.61 & 6.09 & 7.93 & 10.92 \\
\hline \multirow[t]{2}{*}{$C D S$} & & 11.86 & 5.79 & 6.97 & 5.74 & 3.97 & 5.76 & 5.32 & 5.62 \\
\hline & \multicolumn{9}{|c|}{ Panel $C: \mathbb{A}(2,2,2)$} \\
\hline$O I S$ & 8.41 & 3.52 & 9.25 & 9.02 & 5.44 & 3.38 & 2.83 & 4.95 & 8.12 \\
\hline$S P R E A D_{3 M}$ & 6.63 & & 8.40 & 8.78 & 8.64 & 8.15 & 7.47 & 6.61 & 5.58 \\
\hline$S P R E A D_{6 M}$ & & 9.74 & 5.94 & 3.31 & 3.42 & 4.64 & 5.11 & 6.54 & 8.91 \\
\hline$C D S$ & & 11.62 & 4.79 & 6.19 & 4.75 & 3.05 & 5.54 & 3.95 & 3.99 \\
\hline
\end{tabular}

Notes: The table reports root mean squared pricing errors (RMSEs) for each point on the term structures of OIS rates, interest rate spreads, and CDS spreads. SPREAD ${ }_{3 M}$ denotes the difference between the fixed rates on an IRS indexed to 3M EURIBOR and an OIS with the same maturity. SPREAD $6 M$ denotes the difference between the fixed rates on an IRS indexed to $6 \mathrm{M}$ EURIBOR and an OIS with the same maturity. Units are basis points.

Table H.2: Individual RMSEs, EUR market 
Panel A: 1W OIS and Treasury GC repo rates

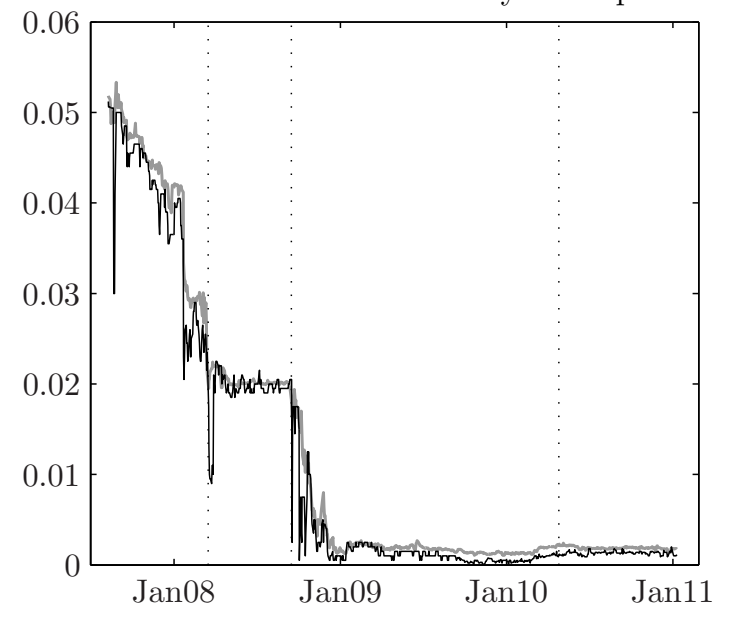

Panel C: 1W OIS and Agency MBS GC repo rates

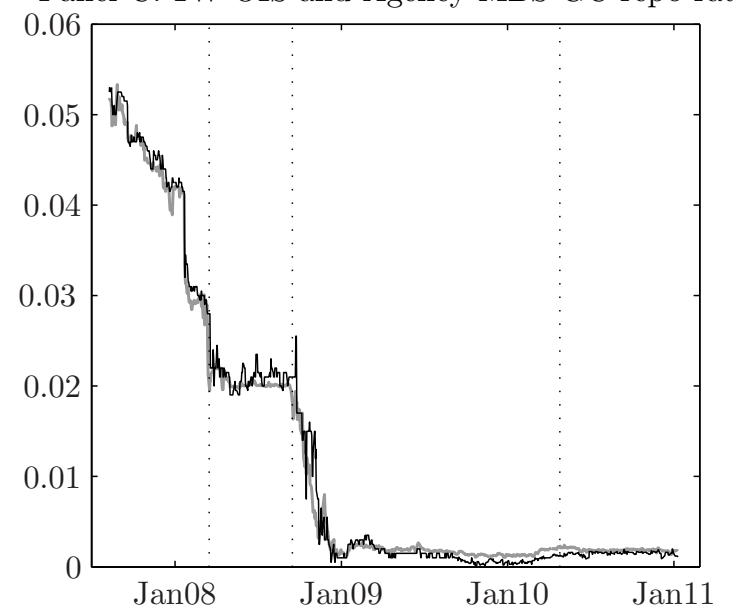

Panel B: 1W OIS and Agency GC repo rates

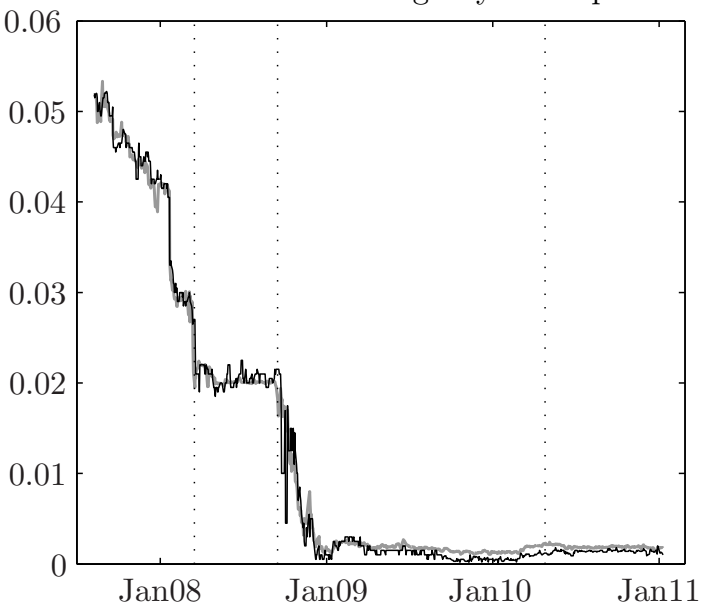

Panel D: 1W EUR OIS and GC repo rates

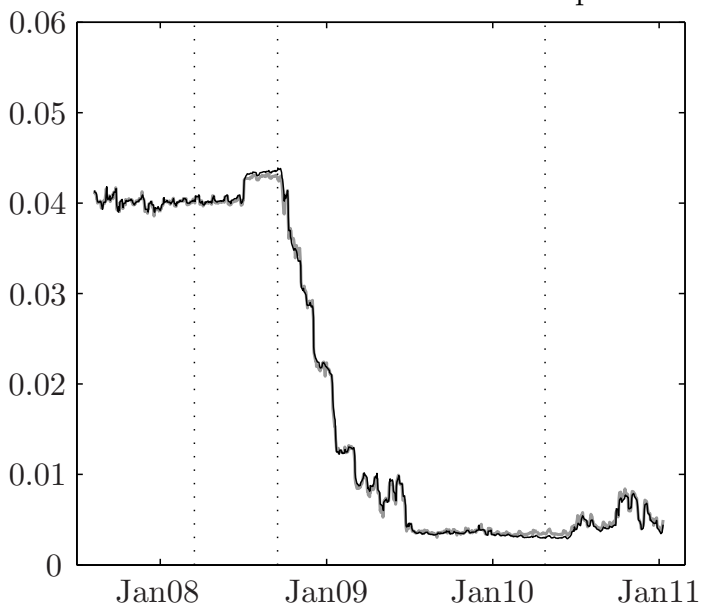

Figure H.1: OIS and repo rates

The figure shows the 1W USD OIS rate along with the $1 \mathrm{~W}$ repo rates for Treasury, Agency, and Agency MBS general collateral (Panels A, B, and C, respectively) as well as the 1W EUR OIS rate along with the 1W Eurepo rate (Panel D). Grey lines mark OIS rates, while black lines mark repo rates. The vertical dotted lines mark the sale of Bear Stearns to J.P. Morgan on March 16, 2008, the Lehman Brothers bankruptcy filing on September 15, 2008, and the downgrade of Greece's debt to non-investment grade status by Standard and Poor's on April 27, 2010. Each time series consists of 895 daily observations from August 09, 2007 to January 12, 2011. 
Panel A: 1W OIS - Treasury GC repo spread

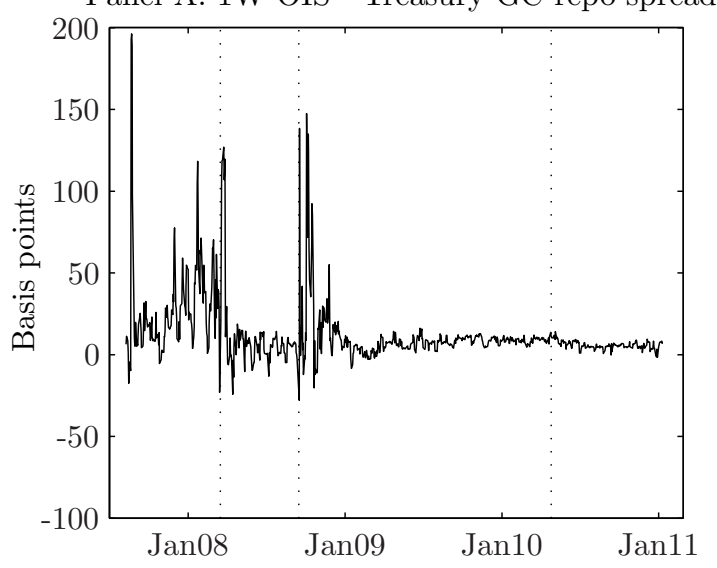

Panel C: 1W OIS - Agency MBS GC repo spread

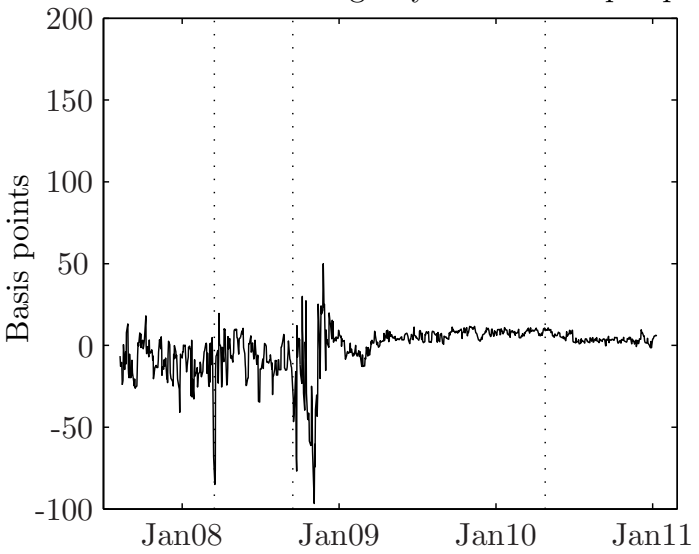

Panel B: 1W OIS - Agency GC repo spread

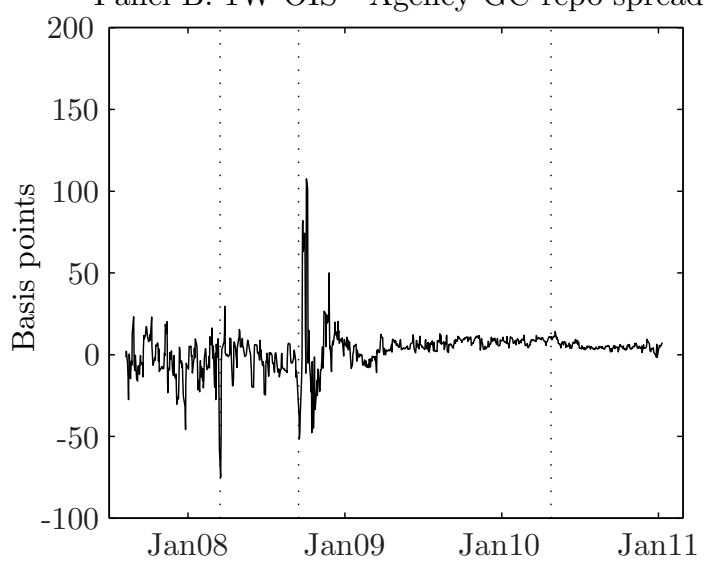

Figure H.2: Spreads between OIS and repo rates

The figure shows the spread between the 1W USD OIS rate and the $1 \mathrm{~W}$ repo rates for Treasury, Agency, and Agency MBS general collateral (Panels A, B, and C, respectively) as well as the spread between the 1W EUR OIS rate and the $1 \mathrm{~W}$ Eurepo rate (Panel D). The vertical dotted lines mark the sale of Bear Stearns to J.P. Morgan on March 16, 2008, the Lehman Brothers bankruptcy filing on September 15, 2008, and the downgrade of Greece's debt to non-investment grade status by Standard and Poor's on April 27, 2010. Each time series consists of 895 daily observations from August 09, 2007 to January 12, 2011. 
$\mathbb{A}(2,1,1)$
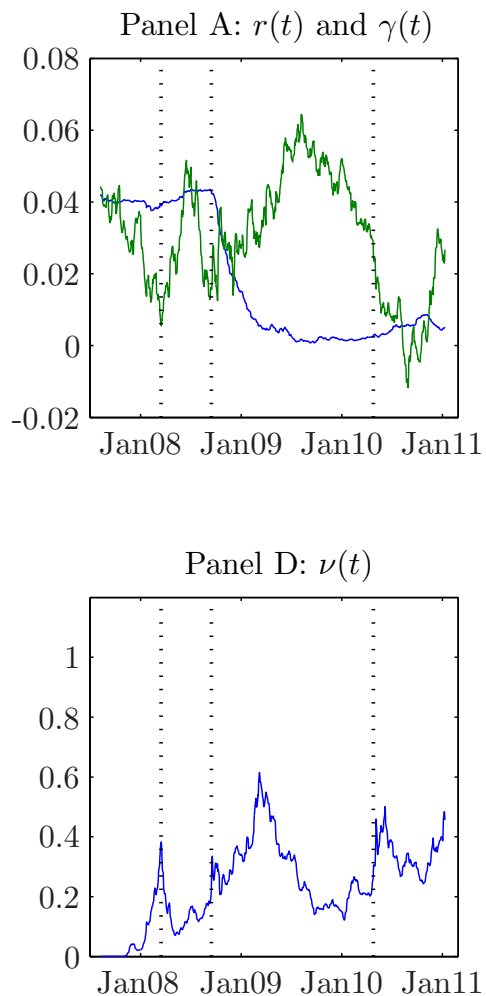

Panel G: $\xi(t)$

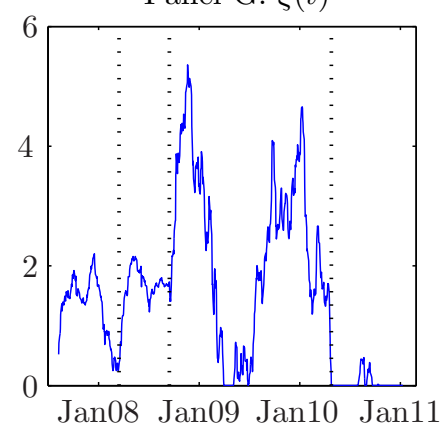

$\mathbb{A}(2,2,1)$

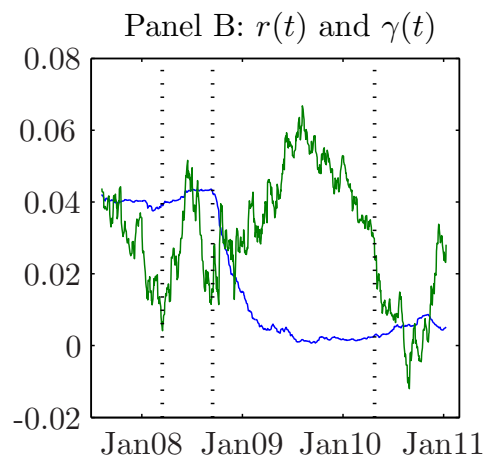

Panel E: $\nu(t)$ and $\mu(t)$

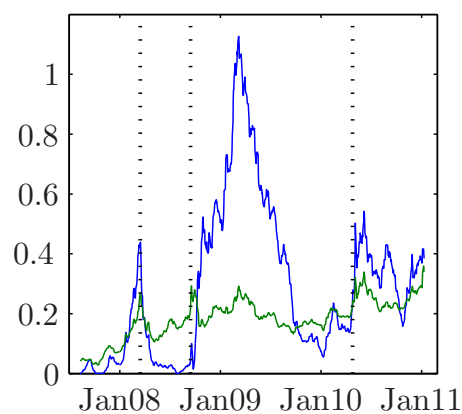

Panel H: $\xi(t)$

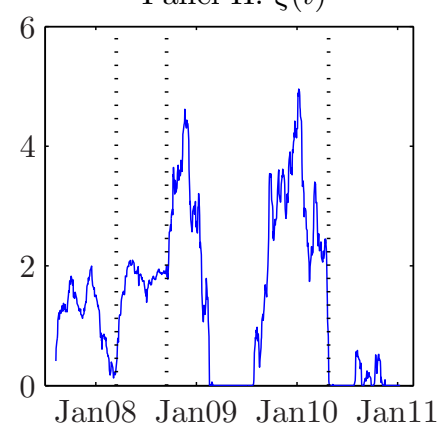

$\mathbb{A}(2,2,2)$

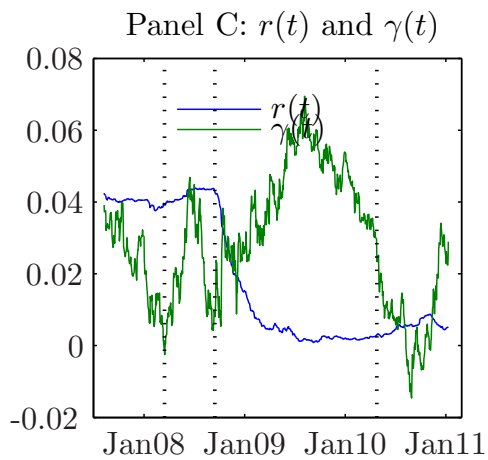

Panel F: $\nu(t)$ and $\mu(t)$

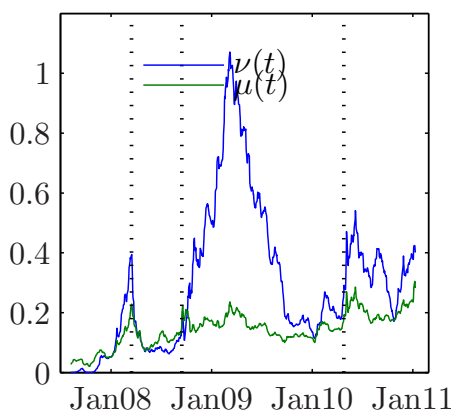

Panel I: $\xi(t)$ and $\epsilon(t)$

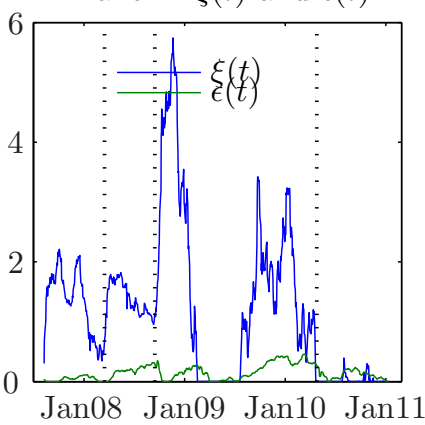

Figure H.3: State variables, EUR

The figure shows the state variables for the three model specifications estimated on EUR data The vertical dotted lines mark the sale of Bear Stearns to J.P. Morgan on March 16, 2008, the Lehman Brothers bankruptcy filing on September 15, 2008, and the downgrade of Greece's debt to non-investment grade status by Standard and Poor's on April 27, 2010. Each time series consists of 895 daily observations from August 09, 2007 to January 12, 2011. 
$\mathbb{A}(2,1,1)$

Panel A1: 3M default prob.

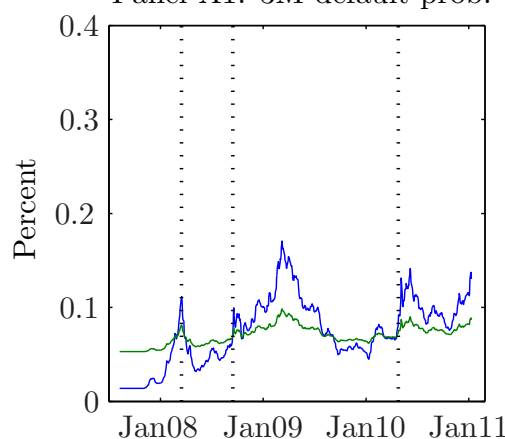

Panel B1: 6M default prob.

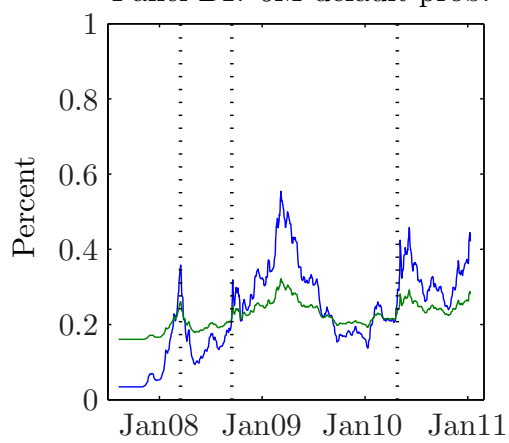

$\mathbb{A}(2,2,1)$

Panel A2: 3M default prob.

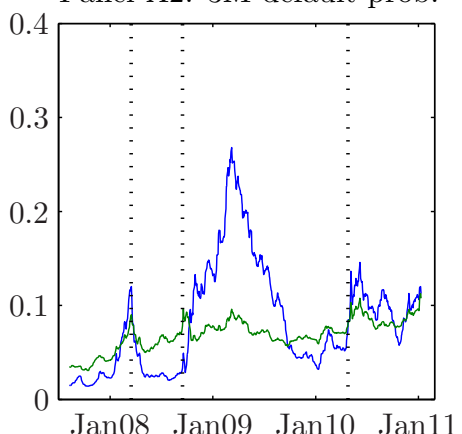

Panel B2: 6M default prob.

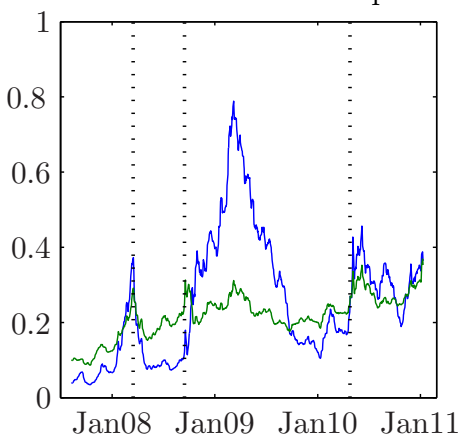

$\mathbb{A}(2,2,2)$

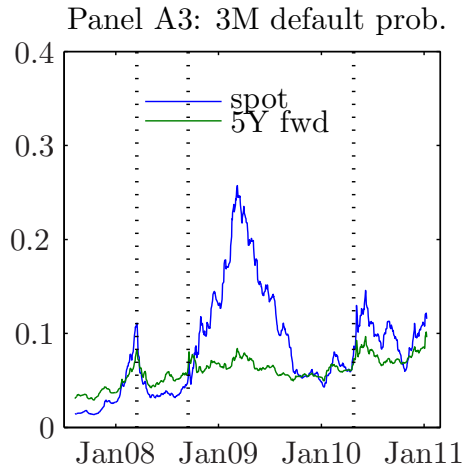

Panel B3: 6M default prob.

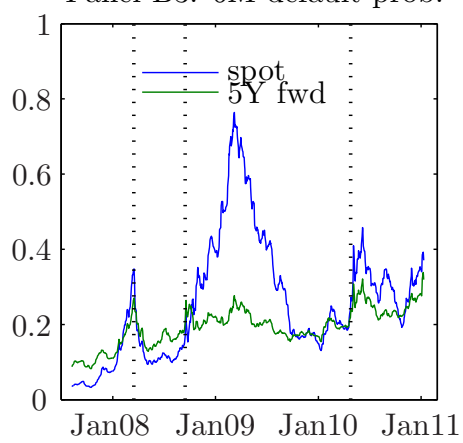

Figure H.4: Default probabilities, EUR

The top panels display the risk-neutral 3M expected default probability (EDP) for an average bank within the current panel as well as for an average bank within the refreshed panel in five year's time. The bottom panels display the corresponding 6M EDPs. The vertical dotted lines mark the sale of Bear Stearns to J.P. Morgan on March 16, 2008, the Lehman Brothers bankruptcy filing on September 15, 2008, and the downgrade of Greece's debt to non-investment grade status by Standard and Poor's on April 27, 2010. Each time series consists of 895 daily observations from August 09, 2007 to January 12, 2011. 

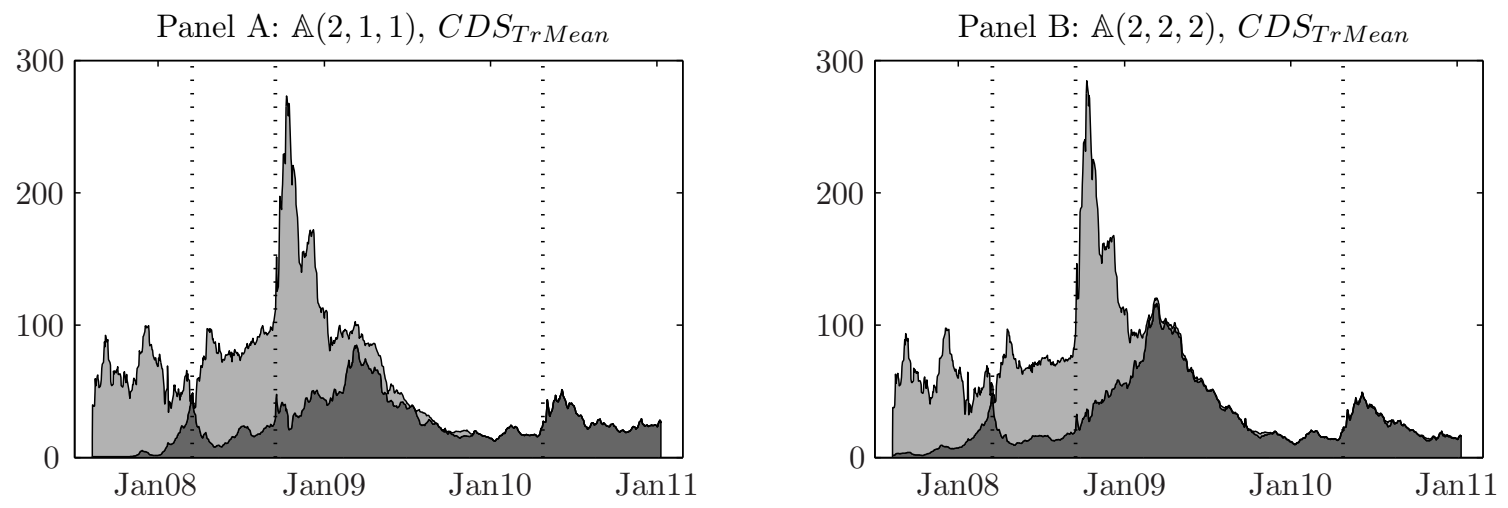

Panel C: $\mathbb{A}(2,2,1)$, stochastic $\Lambda(t), C D S_{\text {TrMean }}$

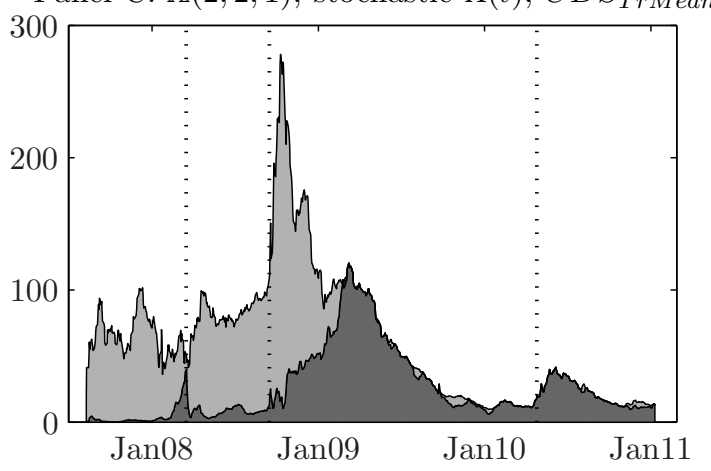

Panel D: $\mathbb{A}(2,2,1)$, no LIBOR, $C D S_{\text {TrMean }}$
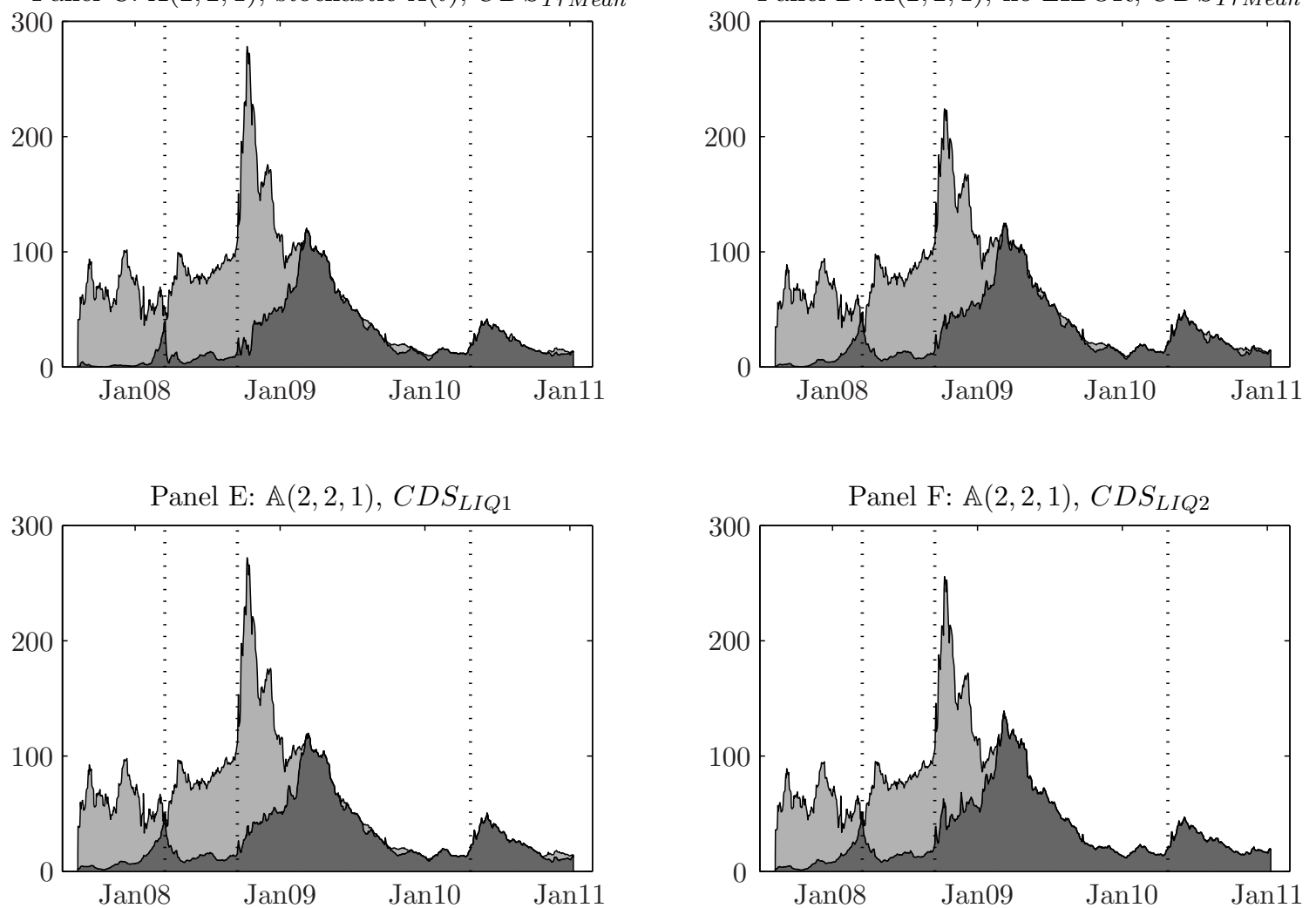

Figure H.5: Alternative decompositions of USD interbank risk, 3M horizon

Alternative decompositions of the 3M LIBOR-OIS spread into default (dark-grey) and non-default (light-grey) components. Panels $\mathrm{A}$ and $\mathrm{B}$ display results using the $\mathbb{A}(2,1,1)$ and $\mathbb{A}(2,2,2)$ specifications, respectively, combined with the $C D S_{\text {TrMean }}$ measure of interbank default risk. Panel C displays results for the $\mathbb{A}(2,2,1)$ specification with a stochastic $\Lambda(t)$, combined with the $C D S_{T r M e a n}$ measure of interbank default risk. Panel D displays results for the $\mathbb{A}(2,2,1)$ specification, combined with the $C D S_{\text {TrMean }}$ measure of interbank default risk, but without using LIBOR rates in the estimation. Panels $\mathrm{E}$ and $\mathrm{F}$ display results using the $\mathbb{A}(2,2,1)$ specification combined with the $C D S_{L I Q 1}$ and $C D S_{L I Q 2}$ measures of interbank default risk, respectively. Units are basis points. The vertical dotted lines mark the sale of Bear Stearns to J.P. Morgan on March 16, 2008, the Lehman Brothers bankruptcy filing on September 15, 2008, æ75d the downgrade of Greece's debt to non-investment grade status by Standard and Poor's on April 27, 2010. Each time series consists of 895 daily observations from August 09, 2007 to January 12, 2011. 

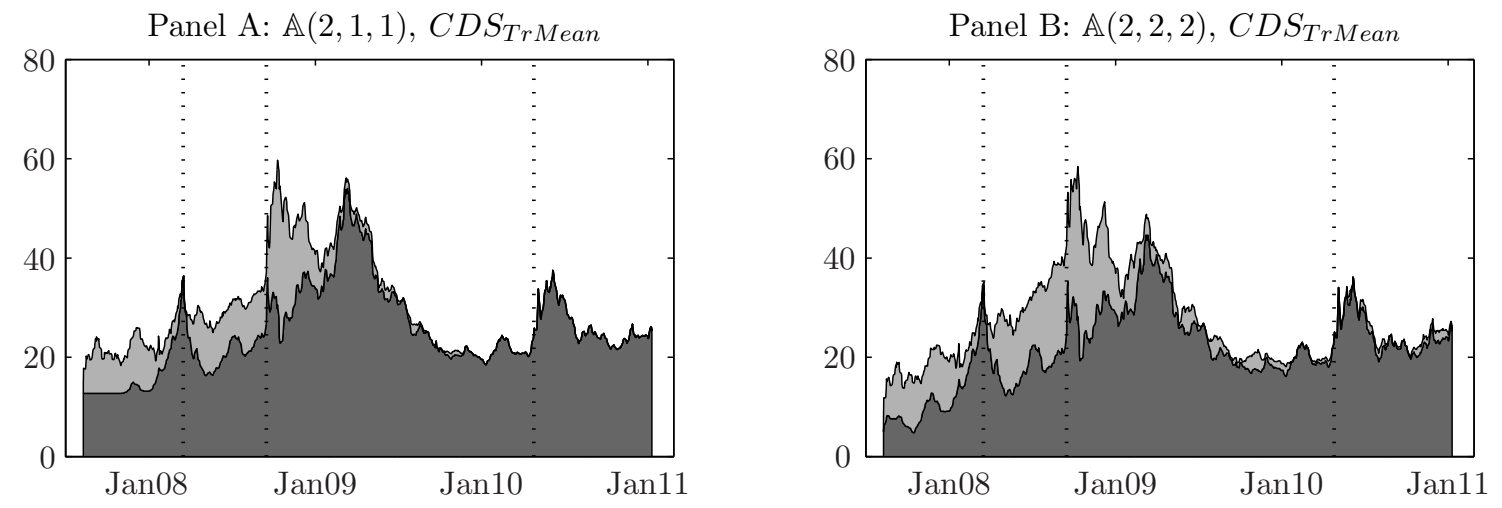

Panel C: $\mathbb{A}(2,2,1)$, stochastic $\Lambda(t), C D S_{\text {TrMean }}$

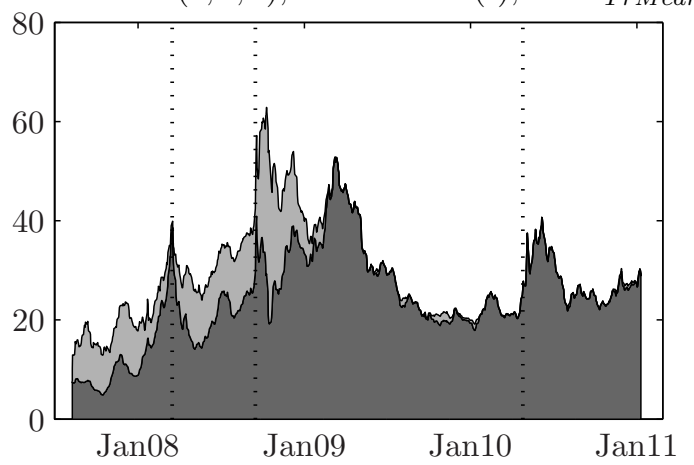

Panel D: $\mathbb{A}(2,2,1)$, no LIBOR, $C D S_{T r M e a n}$

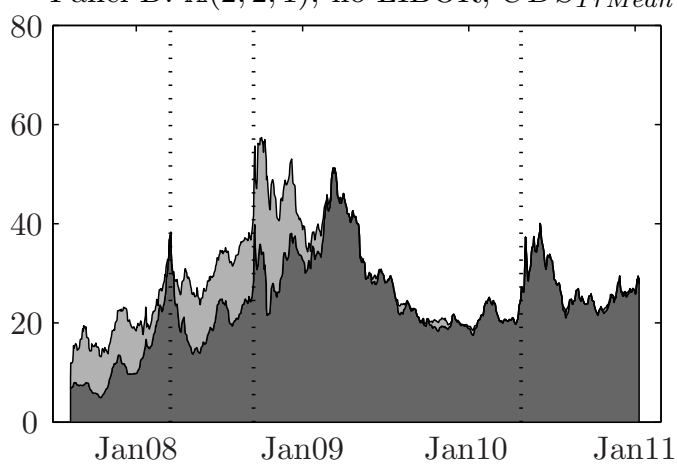

Panel E: $\mathbb{A}(2,2,1), C D S_{L I Q 1}$
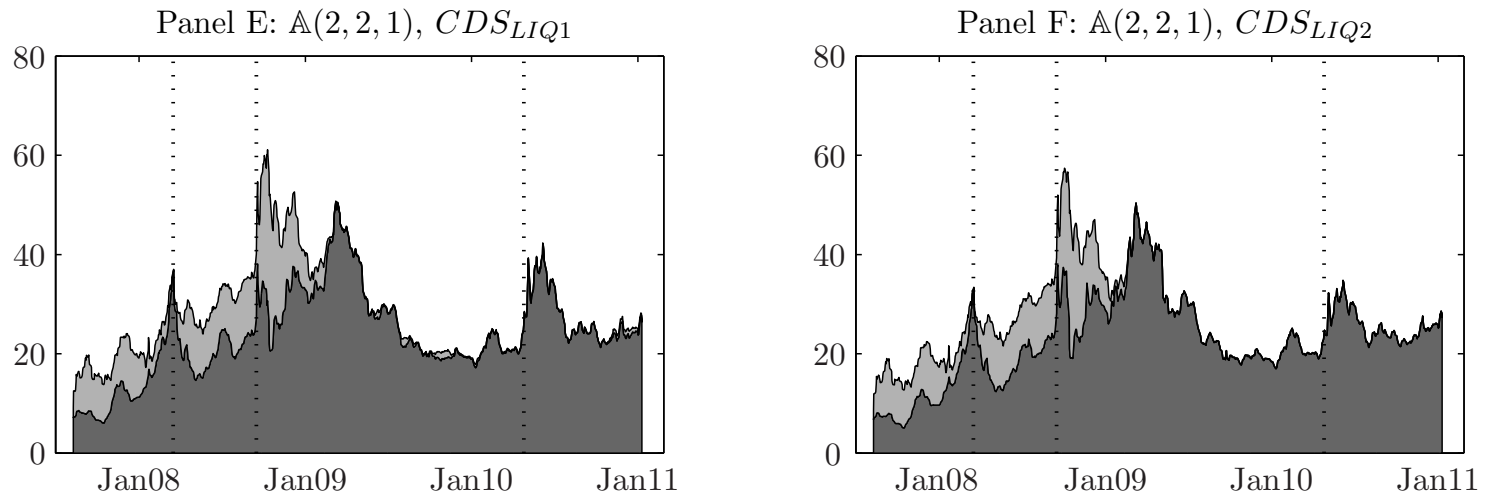

Figure H.6: Alternative decompositions of USD interbank risk, 5Y horizon

Alternative decompositions of the 5Y IRS-OIS spread indexed to 3M LIBOR into default (dark-grey) and nondefault (light-grey) components. Panels $\mathrm{A}$ and $\mathrm{B}$ display results using the $\mathbb{A}(2,1,1)$ and $\mathbb{A}(2,2,2)$ specifications, respectively, combined with the $C D S_{T r M e a n}$ measure of interbank default risk. Panel C displays results for the $\mathbb{A}(2,2,1)$ specification with a stochastic $\Lambda(t)$, combined with the $C D S_{\operatorname{Tr} M e a n}$ measure of interbank default risk. Panel D displays results for the $\mathbb{A}(2,2,1)$ specification, combined with the $C D S_{\text {TrMean }}$ measure of interbank default risk, but without using LIBOR rates in the estimation. Panels $\mathrm{E}$ and $\mathrm{F}$ display results using the $\mathbb{A}(2,2,1)$ specification combined with the $C D S_{L I Q 1}$ and $C D S_{L I Q 2}$ measures of interbank default risk, respectively. Units are basis points. The vertical dotted lines mark the sale of Bear Stearns to J.P. Morgan on March 16, 2008, the Lehman Brothers bankruptcy filing on Septembe§ $\$ 5$, 2008, and the downgrade of Greece's debt to noninvestment grade status by Standard and Poor's on April 27, 2010. Each time series consists of 895 daily observations from August 09, 2007 to January 12, 2011. 